\title{
Propriedades da dinâmica hamiltoniana em níveis de energia convexos de $\mathbb{R}^{4}$
}

\author{
Marcelo Ribeiro de Resende Alves
}

\author{
DissertaÇÃo APRESENTADA \\ $\mathrm{AO}$
}

Instituto de Matemática E Estatística

DA

Universidade De SÃo Paulo

PARA

OBTENÇÃO DO TÍTULO

$\mathrm{DE}$

Mestre em Ciências

Programa: Matemática

Orientador: Prof. Dr. Pedro Antonio Santoro Salomão

Durante o desenvolvimento deste trabalho o autor recebeu auxílio financeiro do CNPq

São Paulo, Abril de 2011 


\section{Propriedades da dinâmica hamiltoniana em níveis de energia convexos de $\mathbb{R}^{4}$}

Esta versão definitiva da dissertação contém as correções e alterações sugeridas pela Comissão Julgadora durante a defesa realizada por Marcelo Ribeiro de Resende Alves em 25/5/2011.

Comissão Julgadora:

- Prof. Dr. Pedro Antonio Santoro Salomão (orientador) - IME-USP

- Prof. Dr. Clodoaldo Grotta Ragazzo - IME-USP

- Prof. Dr. Umberto Leone Hryniewicz - IM-UFRJ 


\section{Agradecimentos}

Ao Professor Pedro pela excelente orientação, atenção, paciência e boas conversas. E também por ter me apresentado a uma área tão rica e ativa.

Aos professores Rosa, Toscano, Clodoaldo, Lucília e Rafael Leão pelas excelentes matérias cursadas ao longo do mestrado. Aos professores Guilherme Antonio, Ilan Grabe e Mario Jorge Dias Carneiro de Belo Horizonte, pelas excelentes aulas e conversas. Ao professor Otani, pelas divertidas conversas sobre música e ciência, e por sua fé inabalável em minhas conjecturas.

À minha família: meu pai, meu irmão e minha avó. Certamente esse trabalho (e tudo mais que faço) seria impossível sem o apoio ao longo de tantos anos.

Aos amigos de BH: Romero (meu futuro colaborador), Dudu, Gugu, Marina Cyrino, Bernardo, Cecília, Rodolfo e pessoal do colégio. Aos colegas e amigos de São Paulo: Naiara e André do IME, Dedé Yokoi, Priscila Pavani, Marina Trombetta, e meus simpáticos vizinhos.

Aos membros da banca, professores Umberto e Clodoaldo, pelo encorajamento e sugestões.

Agradeço também ao pessoal da CPG que me ajudou em momentos tensos. 
"It is better to remain silent and be thought a fool than to open one's mouth and remove all doubt." Abraham Lincoln 


\section{Resumo}

A existência de seções globais para fluxos é de central importância na teoria de sistemas dinâmicos, pois uma seção global simplifica o estudo da dinâmica de um fluxo reduzindo-o ao estudo da dinâmica de um difeomorfismo. Apresentamos detalhadamente a construção devida a Hofer, Zehnder e Wysocki em [17] de uma seção global para o fluxo Hamiltoniano restrito a um nível de energia convexo em $\mathbb{R}^{4}$. Uma importante consequência da existência dessa seção global é que o fluxo Hamiltoniano restrito a um nível de energia convexo em $\mathbb{R}^{4}$ tem 2 ou infinitas órbitas periódicas. Essa construção utiliza-se da teoria de curvas pseudo-holomorfas em simplectizações de variedades de contato desenvolvida pelos mesmos autores em [14], [15] e [16]. Os argumentos apresentados também dão uma nova prova da Conjectura de Weinstein para formas de contato tight em $S^{3}$.

Palavras-chave: níveis de energia convexos, seções globais, curvas pseudo-holomorfas. 


\section{Abstract}

The existence of global surfaces of section to flows is of central importance in the theory of dynamical systems, as a global surface of section simplifies the study of the dynamics of a flow reducing it to study the dynamics of a diffeomorphism. We present in detail the construction due to Hofer, Wysocki and Zehnder in [17] of a global surface of section for the Hamiltonian flow restricted to a convex energy level in $\mathbb{R}^{4}$. An important consequence of the existence of the global surface of section is that the Hamiltonian flow restricted to a convex energy level in $\mathbb{R}^{4}$ has either 2 or infinitely many periodic orbits. This construction utilizes the theory of pseudo-holomorphic curves in symplectizations of contact manifolds developed by the same authors in [14, [15] and [16]. The arguments also give a new proof of Weinstein conjecture for tight contact forms in $S^{3}$.

Keywords: convex energy levels, global surfaces of section, pseudo-holomorphic curves. 


\section{Sumário}

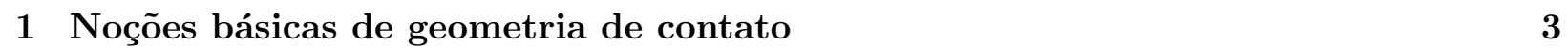

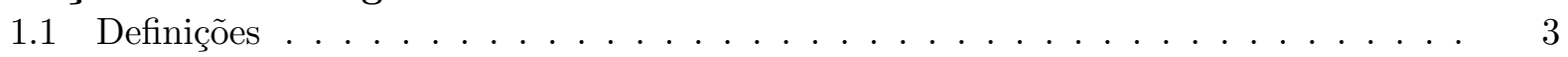

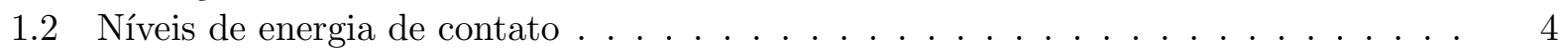

1.3 Exemplos de níveis de contato . . . . . . . . . . . . . . . . . . . . . 4

$1.4 \quad$ Estruturas complexas em $\xi \ldots \ldots \ldots \ldots \ldots \ldots \ldots$

$1.5 \quad$ A estrutura de contato tight em $S^{3} \ldots \ldots \ldots \ldots \ldots \ldots \ldots$

$2 \quad$ O índice de Conley-Zehnder em uma hipersuperfície convexa de $\mathbb{R}^{4} \quad 9$

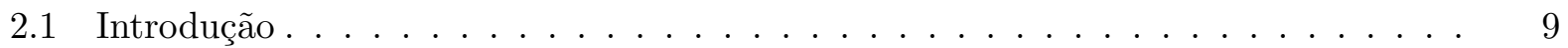

$2.1 .1 \quad$ Caracterização geométrica do índice de Conley-Zehnder . . . . . . . . . . . 9

$2.1 .2 \quad$ Caracterização axiomática do índice de Conley-Zehnder . . . . . . . . . . . 10

2.2 Indice de Conley-Zehnder generalizado f . . . . . . . . . . . . . . . . . 10

2.2 .1 Caso bi-dimensional . . . . . . . . . . . . . . . . . . . . 11

2.2 .2 Caso 2 -dimensional $\ldots \ldots \ldots \ldots \ldots \ldots \ldots$

2.3 Estimativa para o índice de Conley-Zehnder em

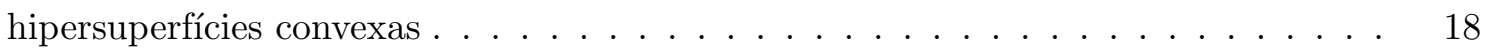

$3 \quad$ Construção do plano de energia finita para formas de contato tight em $S^{3} \quad 23$

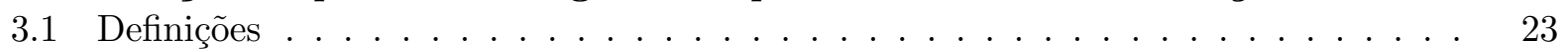

3.2 Comportamento de planos de energia finita $\ldots \ldots \ldots \ldots \ldots \ldots$

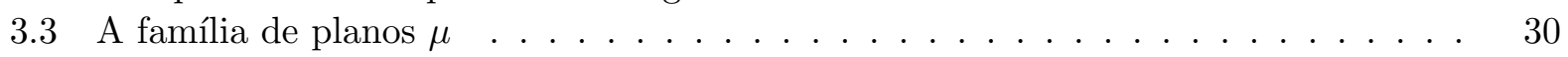

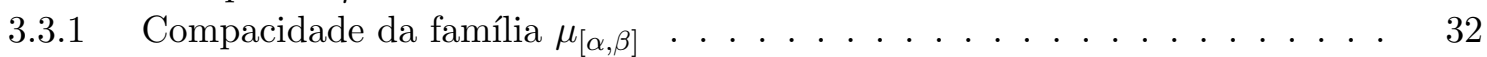

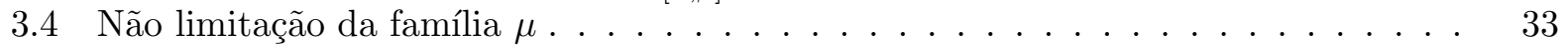

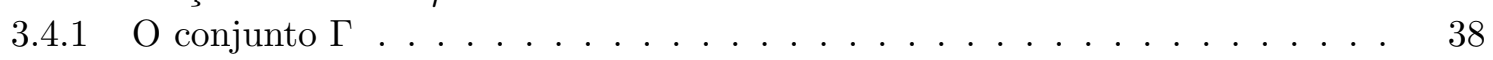

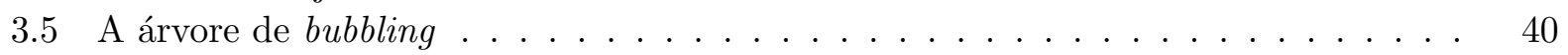

3.6 A decomposição em openbook para níveis de energia convexos . . . . . . . . . . . . 41

\begin{tabular}{|lll|}
\hline 4 & Uma seção global em um nível de energia convexo & 43
\end{tabular}

4.1 Existência da seção global . . . . . . . . . . . . . . . . . . . . . . . 43

4.2 Propriedades e consequências da seção global $\ldots \ldots \ldots$. . . . . . . . . . . . . . 48

\begin{tabular}{|l|l|}
\hline A Propriedades geométricas dos planos pseudo-holomorfos & 51
\end{tabular}

\begin{tabular}{ll}
\hline Referências & 57
\end{tabular} 


\section{Introdução}

O objetivo dessa dissertação é apresentar a construção feita em [17 de um plano de energia finita na simplectização de uma variedade de contato $(M, \lambda)$ onde $M$ é difeomorfa a $S^{3}$ e $\lambda$ é uma forma de contato tight dinamicamente convexa, e suas consequências para a dinâmica do fluxo de Reeb associado. Curvas pseudo-holomorfas na simplectização de variedades de contato foram utilizadas por H. Hofer em [10] para solucionar em vários casos a conjectura de Weinstein (enunciada em [25]), que diz que um fluxo de Reeb em uma variedade de contato tem ao menos uma órbita periódica. Hofer mostrou que a existência de curvas pseudo-holomorfas de energia finita é equivalente à existência de órbitas periódicas para o fluxo de Reeb. Posteriormente, em trabalhos conjuntos com E. Zehnder e K. Wysocki ([14],[15],[16]) foi feito um estudo detalhado sobre propriedades de curvas pseudo-holomorfas de energia finita em simplectizações. Em [17] foi descoberta uma nova aplicação em dinâmica para curvas pseudo-holomorfas em simplectizações, que é a possibilidade de utilizá-las para construir seções globais para fluxos de Reeb em $S^{3}$. Usando essas seções globais foram obtidos importantes resultados sobre a dinâmica de fluxos de Reeb em $S^{3}$.

Daremos agora uma descrição do conteúdo de cada capítulo:

Capítulo 1 - Neste capítulo recordamos as definições básicas de geometria de contato.

Lembramos o que é uma variedade de contato, uma forma de contato $\lambda$, e o campo de Reeb $X$ associado a $\lambda$. Em seguida, provamos as propriedades básicas do fluxo Reeb: preserva a forma de contato $\lambda$ e, consequentemente, a forma volume $\lambda \wedge(d \lambda)^{n}$. Depois, apresentamos a conexão entre campos de Reeb e dinâmica Hamiltoniana: definimos o que é um nível de energia de contato usando campos de Liouville, e mostramos que o fluxo de Reeb sobre este nível de energia de contato é uma reparamentrização do campo Hamiltoniano. Dois exemplos de níveis de energia de contato são exibidos: o fibrado unitário de uma variedade Riemanniana, e uma hipersuperfície starshaped de $\mathbb{R}^{2 n}$. Na última seção do capítulo, construímos a trivialização global e simplética de $\left(\xi^{0}, d \lambda\right)$ onde $\xi^{0}$ é a estrutura de contato tight em $S^{3}$ e $\lambda$ uma forma de contato com $\operatorname{ker} \lambda=\xi^{0}$. Uma trivialização é importante para o cálculo do índice de Conley-Zehnder feita no capítulo seguinte.

Capítulo 2 - O principal resultado deste capítulo é uma estimativa do índice de Conley-Zehnder para órbitas periódicas em níveis de energia convexos: nesses níveis de energia todas as órbitas periódicas tem índice de Conley-Zehnder maior ou igual a 3. Recordamos a definição geométrica e axiomática do índice de Conley-Zehnder e, em seguida, definimos o índice de Conley-Zehnder generalizado e provamos sua coincidência com o índice geométrico no caso não-degenerado. Fazemos uma análise cuidadosa do índice de Conley-Zehnder generalizado no caso multidimensional que é indispensável à estimativa do índice de Conley-Zehnder para níveis de energia convexos.

Capítulo 3 - Este é o principal capítulo da dissertação. Ele contém uma descrição detalhada dos argumentos apresentados em [17] para construir um plano de energia finita para formas de contato tight em $S^{3}$.

Em particular, este argumento dá uma nova prova da conjectura de Weinstein para a formas de contato tight em $S^{3}$. Inicialmente definimos o cobordismo simplético entre $\lambda$ e uma forma de contato especial $\lambda_{E}$. $\lambda_{E}$ é a forma de contato associada a um elipsóide irracional em $\mathbb{R}^{4}$. Usando um resultado de existência de um plano de energia finita no caso da simplectização de $\left(S^{3}, \lambda_{E}\right)$ obtido em [11] é possível arrastar esse plano no cobordismo e gerar um plano de energia finita na simplectização de $\left(S^{3}, \lambda\right)$. Os argumentos de análise de bubbling e compacidade no cobordismo 
são apresentados em detalhe. Em seguida damos uma descrição da árvore de bubbling que aparece como uma consequência dessa construção. Para obter essa descrição nós usamos (sem provar) os resultados de compacidade da chamada "Symplectic Field Theory" que nos dizem como sequências de curvas pseudo-holomorfas no cobordismo se quebram em cilindros, planos e outras curvas pseudoholomorfas que se colam de maneira natural.

Esses resultados de compacidade SFT também são aplicados para o nível de energia convexo. Em particular, é possível dar uma descrição do espaço de planos pseudo-holomorfos que são assintóticos a uma órbita periódica de índice de Conley-Zehnder 3. Esta descrição acaba produzindo uma decomposição em openbook de $S^{3}$ cujas páginas são projeções dos planos de energia finita assintóticos à mesma órbita periódica de índice de Conley-Zehnder 3. Essas páginas são sempre transversais ao campo de Reeb $X_{\lambda}$ fato que será crucial na construção do capítulo seguinte.

Capítulo 4 - Usamos a decomposição em openbook associada a $\lambda$ construída no capítulo anterior para produzir uma seção global para o campo de Reeb. Mostra-se que a dinâmica do campo de Reeb pode ser estudada através da dinâmica de um difeomorfismo do disco que preserva área. Por preservar área esse difeomorfismo tem propriedades especiais como por exemplo a existência de pelo menos um ponto fixo. Um resultado importante de John Franks, diz que se um tal difeomorfismo tiver outro ponto periódico além do ponto fixo ele tem infinitos pontos periódicos. Assim, a existência de uma seção global implica que o fluxo Hamiltoniano em qualquer nível de energia convexo tem 2 ou infinitas órbitas periódicas.

A principal referência para nosso trabalho é o artigo [17] no qual baseamos nossa exposição. 


\section{Capítulo 1}

\section{Noções básicas de geometria de contato}

Nesse capítulo recordamos noções básicas de geometria de contato. Referimos o leitor ao livro [18] para uma exposição mais detalhada desse assunto.

\subsection{Definições}

Uma variedade de contato é um par $\left(M^{2 n+1}, \xi\right)$ onde $M^{2 n+1}$ é uma variedade de dimensão ímpar e $\xi$ é uma distribuição de hiperplanos totalmente não-integrável no sentido de Frobenius. Nessa dissertação estudaremos variedades de contato que possuem a propriedade de que existe uma 1-forma $\lambda$ globalmente definida em $M$, tal que $\xi=\operatorname{ker} \lambda$ (essas são conhecidas na literatura como variedades de contato coorientáveis e $\lambda$ é chamada de forma de contato). A condição de total não-integrabilidade de $\xi$ é equivalente a $\lambda \wedge(d \lambda)^{n} \neq 0$ em todo ponto de $M^{2 n+1}$. Associado à uma variedade de contato temos o chamado campo de Reeb, que é um campo de vetores $X$ em $M^{2 n+1}$ caracterizado pelas seguintes condições:

$$
\begin{gathered}
i_{X(p)} d \lambda=0, \forall p \in M^{2 n+1}, \\
\lambda(X(p))=1, \quad \forall p \in M^{2 n+1} .
\end{gathered}
$$

Note que este campo está unicamente determinado por essas condições.

O fluxo $\phi_{t}$ gerado pelo campo de Reeb é chamado fluxo de Reeb. Duas propriedades importantes do fluxo de Reeb são que ele deixa invariantes a forma de volume $\lambda \wedge(d \lambda)^{n}$ e a estrutura de contato $\xi$. Essas propriedades são consequências diretas da seguinte proposição:

Proposição 1. O fluxo de Reeb preserva a 1-forma de contato $\lambda$.

Demonstração. Pela Fórmula de Cartan, temos:

$$
L_{X} \lambda=i_{X} d \lambda+d i_{X} \lambda=0 .
$$

A derivada de Lie de $\lambda$ com relação a $X$ ser nula implica que $\phi_{t}^{*} \lambda=\lambda$ ou seja o fluxo de Reeb preserva a 1-forma $\lambda$.

Variedades de contato aparecem como níveis de energia de sistemas Hamiltonianos em variedades simplécticas e o fluxo de Reeb é, a menos de uma reparametrização, o fluxo Hamiltoniano restrito a esses níveis de energia de contato. 


\subsection{Níveis de energia de contato}

Considere uma variedade simplética $\left(N=N^{2 n+2}, \omega\right)$ e uma função $H: N \longrightarrow \mathbb{R}$. Seja $N^{\prime} \subset N$ uma hipersuperfície de nível regular. Nesse caso $N^{\prime}$ é invariante pelo fluxo Hamiltoniano gerado pela função $H$ e o campo Hamiltoniano gerado por $H$ não tem singularidades em $N^{\prime}$. O nível de energia $N^{\prime}$ é chamado de contato quando existe um campo de vetores $Y$ definido em uma vizinhança de $N^{\prime}$ satisfazendo a seguinte condição:

$$
L_{Y} \omega=\omega \text { e } Y \text { é transversal à } N^{\prime} .
$$

Nessa situação vale:

Proposição 2. $\left(N^{\prime}, i_{Y} \omega=\lambda\right)$ é uma variedade de contato.

Demonstração. Temos, pela Fórmula de Cartan:

$$
d i_{Y} \omega=L_{Y} \omega-i_{Y} d \omega=L_{Y} \omega=\omega .
$$

Sobre cada ponto $p \in N^{\prime}$ temos que $\omega^{n+1}$ é uma forma de volume em $N$. Tome então uma base de $T_{p} N$ formada por $Y(p)$ e vetores $v_{1}, v_{2} \ldots ., v_{2 n+1}$ tangentes à $N^{\prime}$. Então:

$$
\begin{gathered}
0 \neq \omega^{n+1}\left(Y(p), v_{1}, v_{2} \ldots ., v_{2 n+1}\right)=C i_{Y(p)} \omega \wedge(\omega)^{n}\left(v_{1}, v_{2} \ldots, v_{2 n+1}\right)= \\
=\lambda \wedge(d \lambda)^{n}\left(v_{1}, v_{2} \ldots ., v_{2 n+1}\right) .
\end{gathered}
$$

onde $C \neq 0$ é uma constante. Logo a $2 n+1$-forma $\lambda \wedge(d \lambda)^{n}$ é não-nula em $N^{\prime}$ e é portanto uma forma de volume em $N^{\prime}$. Segue que $\lambda$ é forma de contato em $N^{\prime}$.

Mostraremos agora a relação entre o campo de Reeb $X$ gerado pela 1-forma $\lambda$ e o campo Hamiltoniano $X_{H}$ gerado por $H$ à saber:

Proposição 3. Os campos $X$ e $X_{H}$ tem a mesma direção, ou seja, eles diferem pela multiplicação de uma função não-nula.

Demonstração. Temos:

$$
i_{X_{H}} d \lambda=i_{X_{H}} \omega_{\left.\right|_{N^{\prime}}}=-d H_{\left.\right|_{N^{\prime}}}=0
$$

pois $H$ é constante em $N^{\prime}$.

Como $\lambda$ é forma de contato, existe apenas uma direção tal que os vetores desta direção quando contraídos com $d \lambda$ dão a forma nula, e esta é a direção do campo de Reeb. Como ambos os campos são não nulos segue que $X_{H}$ tem a mesma direção do campo de Reeb.

\subsection{Exemplos de níveis de contato}

Nesta seção mostraremos diversos exemplos importantes na literatura de níveis de energia de contato.

\section{Hipersuperfícies starshaped em $\mathbb{R}^{2 n}$}

Considere $\mathbb{R}^{2 n}$ com a forma simplética canônica $\omega_{0}=\sum_{i=1}^{n} d x_{i} \wedge d y_{i}$. Tome em $\mathbb{R}^{2 n}$ o campo radial $Y(x, y)=\frac{(x, y)}{2}$. Então Y é uma campo de Liouville pois:

$$
L_{Y} \omega_{0}=i_{Y} d \omega_{0}+d i_{Y} \omega_{0}=d\left(i_{Y} \sum_{i=1}^{n} d x_{i}\right)=d\left(\frac{1}{2} \sum_{i=1}^{n} x_{i} d y_{i}-y_{i} d x_{i}\right)=\omega_{0} .
$$


Uma hipersuperfície compacta e sem bordo $S$ é chamada starshaped em relação à origem quando: toda semi-reta começando na origem intersecta $S$ em um único ponto transversalmente. Observamos que essas hipersuperfícies são difeomorfas a $S^{2 n-1}$ e transversais à $Y$.

Como hipersuperfícies transversais a $\mathrm{Y}$ tem uma estrutura de contato, as hipersuperfícies starshaped com relação à origem são, portanto, de contato.

\section{Fluxo geodésico}

O fluxo geodésico descreve as trajetórias inerciais de partículas sobre uma variedade Riemanniana $(M, g)$. Ele pode ser descrito como o fluxo Lagrangeano no fibrado tangente $T M$ de $M$, associado ao funcional $\int_{a}^{b}\langle\dot{\gamma}, \dot{\gamma}\rangle_{g}$ onde $\gamma:[a, b] \longrightarrow M$ e $-\infty<a<b<+\infty$ (ver [24]). Usando a transformada de Legendre é possível descrever o fluxo geodésico como um fluxo Hamiltoniano em $T^{*} M$ com a estrutura simplética canônica de $T^{*} M$ (que definiremos abaixo). Tomando $(q, p) \in T^{*} M$ com $q \in M$ e $p$ um funcional linear em $T_{q} M$ a função Hamiltoniana para o fluxo geodésico de $(M, g)$ é:

$H(q, p)=g_{q}\left(v_{p}, v_{p}\right)$ onde $v_{p}$ é o único vetor de $T_{q} M$ tal que $g_{q}\left(v_{p},.\right)=p($.$) .$

Para definir a estrutura simplética canônica em $T^{*} M$ definimos inicialmente a 1-forma tautológica $\alpha$ em $T^{*} M$. Dado $(q, p) \in T^{*} M$ e $w \in T_{(q, p)} T^{*} M$ definimos:

$\alpha(w)=p(d \pi w)$ onde $\pi: T^{*} M \rightarrow M$ é a projeção canônica de $T^{*} M$ em $M$.

Introduzindo coordenadas cotangentes $(q, p)=\left(q_{1}, \ldots, q_{n}, p_{1}, \ldots, p_{n}\right)$ numa vizinhança de um ponto $q$ em $M$, a 1-forma tautológica se escreve como:

$$
\alpha=\sum_{i=1}^{n} p_{i} d q_{i}
$$

Isso implica que a 2-forma $\omega_{0}:=d \alpha=\sum_{i=1}^{n} d p_{i} \wedge d q_{i}$ é uma forma simplética em $T^{*} M$ pois nessas coordenadas vemos que ela é fechada e não-degenerada. É com relação à $\omega_{0}$ que tomamos o gradiente simplético de $H$ acima para definir o fluxo geodésico.

Mostraremos agora que o nível de energia $H^{-1}(1)$ é um nível de energia de contato. Inicialmente considere o campo de vetores $Y(q, p)$ que em coordenadas locais se expressa como $Y(q, p)=\left(0, \ldots 0, p_{1}, \ldots, p_{n}\right)$. Esse campo tem uma caracterização independente de coordenadas como o campo radial em cada fibra e, portanto, ele está bem definido em todo $T^{*} M$. Temos que $Y$ é um campo de Liouville pois:

$$
L_{Y} \omega_{0}=i_{Y} d \omega_{0}+d i_{Y} \omega_{0}=d\left(i_{Y} \sum_{i=1}^{n} d p_{i} \wedge d q_{i}\right)=d \sum_{i=1}^{n} p_{i} d q_{i}=\omega_{0} .
$$

Como $H^{-1}(1)$ é transversal ao campo $Y$ temos então que o fluxo Hamiltoniano de $H^{-1}(1)$ é uma reparametrização do fluxo de Reeb de $\alpha$ em $H^{-1}(1)$.

\subsection{Estruturas complexas em $\xi$}

Nos próximos capítulos estudaremos curvas pseudo-holomorfas para certas estruturas quasecomplexas em $\mathbb{R} \times S^{3}$. Essas estruturas quase-complexas tem a propriedade de deixar a estrutura de contato $\xi$ invariante. Na verdade observamos que $\left(\xi,\left.d \lambda\right|_{\xi}\right)$ é um fibrado vetorial simplético sobre $S^{3}$. É um resultado de Gromov [8 que dado um fibrado vetorial simplético, é possível achar uma estrutura complexa suave $J$ nesse fibrado que é compatível com a forma simplética $d \lambda$ em cada 
fibra no sentido que $d \lambda(\cdot, J \cdot)$ é uma aplicação bilinear simétrica positiva-definida. Referimos a [18] para uma demonstração deste fato.

\subsection{A estrutura de contato tight em $S^{3}$}

Nesta seção daremos uma descrição de propriedades da estrutura de contato tight de $S^{3}$ (é um resultado de Eliashberg que $S^{3}$ admite apenas uma estrutura de contato tight módulo difeomorfismos). Essa estrutura de contato é a obtida considerando em $S^{3}=\left\{x \in \mathbb{R}^{4},|x|=1\right\}$ a restrição da 1-forma $\lambda_{0}=\sum_{n=1}^{2} \frac{p_{i} d q_{i}-q_{i} d p_{i}}{2}$ (considerando em $\mathbb{R}^{4}$ coordenadas $\left(p_{1}, q_{1}, p_{2}, q_{2}\right)$ ).

Já vimos na seção 1.3 que $\left(S^{3}, \lambda_{0}\right)$ é uma variedade de contato. Daremos agora uma nova prova desse fato.

Seja $x \in S^{3}$ e considere $i, j$ e $k$ os quatérnions que são canonicamente chamados assim. Então $\{x, i x, j x, k x\}$ é uma base ortonormal de $T_{x} \mathbb{R}^{4} \cong \mathbb{R}^{4}$. Como $x \perp T_{x} S^{3}$, temos que $\{i x, j x, k x\}$ é uma base ortonormal de $T_{x} S^{3}$. Chamando $\lambda=\left.\lambda_{0}\right|_{S^{3}}$ e lembrando que $d \lambda_{0}=\sum_{i=1}^{2} d p_{i} \wedge d q_{i}=\omega_{0}$, onde $\omega_{0}$ é a forma simplética canônica de $\mathbb{R}^{4}$, calculamos:

$$
d \lambda(j x, k x)=d \lambda_{0}(j x, k x)=\omega_{0}(j x, k x)=\langle i j x, k x\rangle=\langle k x, k x\rangle=|x|=1,
$$

o que implica que o núcleo de $d \lambda$ é unidimensional. Além disso, como núcleo de $\lambda$ em $T_{x} S^{3}$ é gerado por $i x$, temos que os núcleos de $d \lambda$ e $\lambda$ são disjuntos. Isso é o suficiente para garantir que $\lambda \wedge d \lambda \neq 0$ e, portanto, $\lambda$ é forma de contato. Observamos que durante essa prova, construímos uma trivialização global e simplética da estrutura de contato $\xi^{0}=\operatorname{ker}(\lambda)$, vendo $\left(\xi^{0},\left.d \lambda\right|_{\xi^{0}}\right)$ como um fibrado vetorial simplético. Para escrever essa trivialização como uma aplicação $\Phi: \xi^{0} \longrightarrow S^{3} \times\left(\mathbb{R}^{2}, \omega_{0}\right)$ fazemos:

$$
\begin{array}{r}
\Phi(x, j x)=\left(x, e_{1}\right) \in S^{3} \times \mathbb{R}^{2}, \\
\Phi(x, k x)=\left(x, e_{2}\right) \in S^{3} \times \mathbb{R}^{2}, \\
\Phi(x, \cdot) \text { é linear de } \xi_{x}^{0} \text { em }\{x\} \times \mathbb{R}^{2} .
\end{array}
$$

Para ver que $\Phi$ é uma trivialização simplética observe que ela leva uma base simplética de $\left(\xi_{x}^{0}, d \lambda_{x}\right)$ em uma base simplética de $\{x\} \times\left(\mathbb{R}^{2}, \omega_{0}\right)$.

Existe um método para gerar a partir de uma forma de contato $\lambda$ todas as outras formas de contato associadas à estrutura de contato ker $\lambda$, que é multiplicar $\lambda$ por uma função $f$ que nunca se anule. Assumiremos sem perda de generalidade que $f$ é positiva. Isso permite que construamos para todas as formas de contato $f \lambda$ em $S^{3}$ trivializações globais e simpléticas do fibrado vetorial simplético $\left(\xi^{0}, d\left(\left.f \lambda\right|_{\xi^{0}}\right)\right)$. Tome $\{j x, k x\}$ a base construída para $\xi_{x}^{0}$. Calculamos então:

$$
d(f \lambda)(j x, k x)=(f d \lambda+d f \wedge \lambda)(j x, k x)=(f d \lambda)(j x, k x)=f(x),
$$

onde na segunda igualdade usamos que $\{j x, k x\} \in \operatorname{ker} \lambda$. Então $\left\{\frac{j x}{\sqrt{f(x)}}, \frac{k x}{\sqrt{f(x)}}\right\}$ é uma base simplética de $\left(\xi_{x}^{0}, d\left(f \lambda_{x}\right)\right)$. Definimos então:

$$
\begin{gathered}
\Phi\left(x, \frac{j x}{\sqrt{f(x)}}\right)=\left(x, e_{1}\right) \in S^{3} \times \mathbb{R}^{2}, \\
\Phi\left(x, \frac{k x}{\sqrt{f(x)}}\right)=\left(x, e_{2}\right) \in S^{3} \times \mathbb{R}^{2}, \\
\Phi(x, \cdot) \text { é linear de } \xi_{x}^{0} \text { em }\{x\} \times \mathbb{R}^{2},
\end{gathered}
$$

que é uma trivialização global e simplética de $\left(\xi^{0}, d(f \lambda)\right)$.

Iremos agora mostrar como através de uma hipersuperfície starshaped com relação à origem de $\mathbb{R}^{4}$ calcular a forma de contato que ela induz em $S^{3}$. 
Seja $S$ hipersuperfície starshaped e defina em $S^{3}$ a seguinte função. Dado $p \in S^{3}$ seja $F(p)$ o único número positivo tal que $F(p) p \in S$. Definimos então a aplicação $G: S^{3} \longrightarrow S$ por $G(p)=F(p) p$. O fato de $S$ ser starshaped implica que $G$ é bijetora e, suave quando $S$ é suave, pois ela é a inversa da aplicação suave $\frac{x}{|x|}$ de $S$ em $S^{3}$. Notamos que é possível estender $G$ a uma aplicação $\widetilde{G}: \mathbb{R}^{4} \backslash\{0\} \rightarrow \mathbb{R}^{4} \backslash\{0\}$ dada por $\widetilde{G}(p)=F\left(\frac{p}{|p|}\right) p$. Para ver que $G$ é um difeomorfismo calcula-se que para $p \in S^{3}$ temos $\operatorname{det}(D \widetilde{G})=F^{4}$.

Vimos que $\lambda$ restrita a $S$ é uma forma de contato em $S$. Gostaríamos de escrever explicitamente essa forma em $S^{3}$. Calculamos inicialmente $\left(G^{*} \lambda\right)$ no ponto $p_{0}=(1,0,0,0)\left(\operatorname{denotamos}\left(G^{*} \lambda\right)_{p}\right.$ a forma $\left(G^{*} \lambda\right)$ no ponto $\left.p\right)$. Para isso notamos que um cálculo direto nos dá:

$$
G^{*} \lambda_{p_{0}}=\left(F\left(p_{0}\right)\right)^{2} \lambda\left(p_{0}\right) .
$$

Para tratar do caso geral inicialmente observamos para cada $p \in S^{3}$ o difeomorfismo linear $h_{p}$ de $\mathbb{R}^{4}$ em $\mathbb{R}^{4}$ obtido multiplicando cada elemento de $\mathbb{R}^{4}$ pelo quatérnion $p$ preserva a 1-forma $\lambda$. Além disso ele também preserva $S^{3}$, e leva $p_{0}$ em $p$. Definimos então a aplicação $G_{p}: S^{3} \longrightarrow S$ por $\left(h_{p}\right)^{-1} \circ G \circ h_{p}$. O difeomorfismo $G_{p}$ então se escreve como : $G_{p}(x)=F\left(h_{p}(x)\right) x$. Então por um lado calculamos:

$$
G_{p}^{*} \lambda_{p_{0}}=(F(p))^{2} \lambda_{p_{0}}
$$

Usando essa equação temos:

$$
\begin{gathered}
\left.(F(p) \lambda)_{p}=\left(h_{p}^{-1}\right)^{*}(F(p) \lambda)_{p_{0}}=\left(h_{p}^{-1}\right)^{*}\left(G_{p}\right)^{*} \lambda\right)_{p_{0}}= \\
=\left(\left(h_{p}^{-1}\right)^{*}\left(h_{p}\right)^{*}(G)^{*}\left(h_{p}^{-1}\right)^{*} \lambda\right)_{p_{0}}=G^{*}\left(\left(h_{p}\right)^{*} \lambda\right)_{p_{0}}=G^{*} \lambda_{p},
\end{gathered}
$$

e obtemos que $G^{*} \lambda=(F(p))^{2} \lambda$.

O último resultado que provaremos nessa seção é a construção para cada hipersuperfície starshaped $S$, de uma função Hamiltoniana que tem $S$ como nível de energia e cujo fluxo Hamiltoniano nesse nível coincide com o fluxo de Reeb de $\lambda_{0}$ restrita a $S$.

Essa função será chamada de $H$ e tem as seguintes propriedades:

$$
\begin{aligned}
& H^{-1}(1)=S, \\
& H(t x)=t^{2} H(x), \forall x \in \mathbb{R}^{4}, t \in \mathbb{R} .
\end{aligned}
$$

Já vimos que o fluxo Hamiltoniano de $H$ e o fluxo de Reeb de $(S, \lambda)$ diferem por uma reparametrização. Logo, como seus campos são linearmente dependentes, para mostrar que eles são idênticos basta provar que $\lambda\left(X_{H}\right)=1$ onde $X_{H}$ é o campo Hamiltoniano de $H$. De fato, temos:

$$
\lambda\left(X_{H}(x)\right)=\left(i_{\frac{x}{2}} \omega_{0}\right)\left(X_{H}(x)\right)=\omega_{0}\left(\frac{x}{2}, X_{H}(x)\right)=\frac{1}{2} d H_{x}(x) .
$$

Usando homogeneidade de $H$ temos:

$$
d H_{x}(x)=\left.\frac{\partial}{\partial t} H((1+t) x)\right|_{t=0}=\left.\frac{\partial}{\partial t}\left((1+t)^{2} H(x)\right)\right|_{t=0}=2 H(x) .
$$

Se $x \in S$, temos $H(x)=1$ e, portanto, $\lambda\left(X_{H}(x)\right)=\frac{1}{2}(2 H(x))=1$. Isso prova que os dois campos são idênticos. 


\section{Capítulo 2}

\section{$\mathrm{O}$ índice de Conley-Zehnder em uma hipersuperfície convexa de $\mathbb{R}^{4}$}

\subsection{Introdução}

Considere a variedade de contato $\left(S^{3}, \lambda\right)$ onde $\lambda$ é forma de contato tight com ker $\lambda=\xi^{0}$. Segue que $\left(\xi^{0},\left.d \lambda\right|_{\xi^{0}}\right)$ é um fibrado vetorial simplético, e como vimos no capítulo anterior é possível construir uma trivialização global e simplética $\Psi: \xi^{0} \longrightarrow S^{3} \times \mathbb{R}^{2}$ de $\left(\xi^{0},\left.d \lambda\right|_{\xi^{0}}\right)$, onde em $\mathbb{R}^{2}$ consideramos a forma simplética canônica $\omega_{0}$.

O índice de Conley-Zehnder mede, a grosso modo, o giro do fluxo de Reeb linearizado ao longo de uma órbita periódica. O principal resultado deste capítulo é uma estimativa para o índice de Conley-Zehnder de órbitas periódicas para formas de contato que são induzidas em $S^{3}$ por hipersuperfícies convexas de $\mathbb{R}^{4}$.

\subsubsection{Caracterização geométrica do índice de Conley-Zehnder}

Seja $P(t)$ uma órbita periódica não-degenerada de período $T$ do fluxo de Reeb $\phi_{t}$ associado à $\lambda$ (dizemos que uma órbita é não-degenerada quando $\left.D \phi_{T}\right|_{\xi^{0}}(P(0)): \xi_{P(0)}^{0} \rightarrow \xi_{P(0)}^{0}$ não tem 1 como autovalor). Como vimos anteriormente $D \phi_{t}(P(0))$ é uma aplicação linear simplética de $\xi_{P(0)}^{0} \mathrm{em}$ $\xi_{P(t)}^{0}$, e usando a trivialização $\Psi$ associamos ao caminho de aplicações $\left\{D \phi_{t}(P(0)), t \in \mathbb{R}\right\}$ um caminho de simplectomorfismos de $\mathbb{R}^{2}$ definido por:

$$
\Psi\left(P(t), D \phi_{t}(P(0)) \circ \Psi^{-1}(P(0), \cdot)\right): \mathbb{R}^{2} \longrightarrow \mathbb{R}^{2} .
$$

A esse caminho associamos um caminho de matrizes simpléticas $A(t)$ em $S p(1)$, usando a representação de $\Psi\left(P(t), D \phi_{t}(P(0)) \circ \Psi^{-1}(P(0), \cdot)\right)$ na base canônica de $\mathbb{R}^{2}$. Consideremos agora, para cada $v \neq 0 \in \mathbb{R}^{2}$ o caminho $A(t) v$. Como esse caminho nunca passa pela origem, é possível associar a ele um argumento contínuo (normalizado a 1) $\arg _{v}(t)$. Chamamos $\triangle(v)$ a variação desse argumento entre 0 e $T$. Observamos que se 2 vetores $v$ e $w$ não nulos são linearmente dependentes então eles tem a mesma variação de argumento. Definimos:

$$
I(A)=\left\{\triangle(v), v \in \mathbb{R}^{2} \text { e } v \neq 0\right\} .
$$

É possível provar que $I(A)$ é um intervalo de comprimento menor que $\frac{1}{2}$. O índice de ConleyZehnder é definido como:

$$
\left\{\begin{array}{l}
\mu_{C Z}(P)=2 k+1 \text { se } I(A) \subset(k, k+1), \\
\mu_{C Z}(P)=2 k \text { se } k \in I(A) .
\end{array}\right.
$$

Essa definição captura o giro médio de $D \phi_{t}(P(0))$ em relação à trivialização $\Psi$. O índice de Conley-Zehnder pode ser generalizado para caminhos de matrizes simpléticas em $S p(n)$. A caracterização axiomática abaixo mostra uma maneira como isso pode ser feito. 


\subsubsection{Caracterização axiomática do índice de Conley-Zehnder}

Em [15] é provado que o índice de Conley-Zehnder pode ser caracterizado axiomaticamente. Seja $G(n)$ o conjunto de arcos simpléticos fechados em $S p(n)$ e $m^{n}: G(n) \longrightarrow \mathbb{Z}$ o índice de Maslov. Seja $\Sigma(n)$ o conjunto dos arcos não-degenerados (a matriz $A(T)$ não tem autovalor 1) $A(t)$ em $S p(n)$ com $A(0)=I d$. Então, existe apenas uma família de aplicações $\mu_{C Z}^{n}: \Sigma(n) \longrightarrow \mathbb{Z}$ satisfazendo:

- $\mu_{C Z}^{n+m}(A \oplus B)=\mu_{C Z}^{n}(A)+\mu_{C Z}^{m}(B), \forall A \in \Sigma(n), B \in \Sigma(m)$.

- $\mu_{C Z}^{n}(g A)=2 m^{n}(g)+\mu_{C Z}^{n}(A), \forall A \in \Sigma(n), g \in G(n)$.

- $\mu_{C Z}^{n}(A)=-\mu_{C Z}^{n}\left(A^{-1}\right)$.

- $\mu_{C Z}^{1}\left(A_{0}\right)=1$ se $A_{0}(t)=e^{i \pi t} ; t \in[0,1]$.

Nesse caso $\mu_{C Z}^{1}$ coincide com o índice de Conley-Zehnder geométrico. Além disso, $\mu_{C Z}^{n}$ classifica completamente os elementos de $\Sigma(n)$ a menos de homotopia, ou seja:

$$
\mu_{C Z}^{n}\left(A_{1}\right)=\mu_{C Z}^{n}\left(A_{2}\right) \Leftrightarrow A_{1} \text { e } A_{2} \text { são homotópicos em } \Sigma(n) \text {. }
$$

\section{$2.2 \quad$ Índice de Conley-Zehnder generalizado}

Nessa subseção daremos mais uma forma de caracterizar o índice de Conley-Zehnder, desta vez usando a ordenação do espectro de certos operadores auto-adjuntos, que foi introduzida em [15]. Essa caracterização será essencial para provar a estimativa prometida em nossa introdução e além disso tem a vantagem de também estar definida para órbitas periódicas degeneradas.

Iniciamos com a seguinte observação. Dado um arco $A(t) \in \Sigma(n) \operatorname{com} A(0)=I d$ associamos um caminho de matrizes simétricas dado por:

$$
S(t)=-J \dot{A}(t) A^{-1}(t),
$$

onde $J$ é a estrutura complexa usual de $\mathbb{R}^{2 n}=\mathbb{C}^{n}$. Se utilizarmos coordenadas $\left(q_{1}, p_{1}, \ldots ., q_{n}, p_{n}\right)$ em $\mathbb{R}^{2 n}$ temos que $J$ é caracterizada por: $J \partial_{q_{i}}=\partial_{p_{i}}, J \partial_{p_{i}}=-\partial_{q_{i}}$ e $J^{2}=-I d$. Nesse caso temos que $A(t)$ pode ser caracterizado como a única solução do problema de valor inicial:

$$
\begin{aligned}
& \dot{A}(t)=J S(t) A(t), \\
& A(0)=I d .
\end{aligned}
$$

Reciprocamente, dado um caminho de matrizes simétricas $S(t)$, o caminho $A(t)$ que satisfaz:

$$
\begin{aligned}
& \dot{A}(t)=J S(t) A(t), \\
& A(0)=I d .
\end{aligned}
$$

é necessariamente de matrizes simpléticas.

Logo, existe uma relação biunívoca entre arcos de matrizes simpléticas e arcos de matrizes simétricas. Para definição do índice de Conley-Zehnder generalizado usaremos arcos de matrizes simétricas, e através da relação acima exposta definiremos o índice generalizado do arco de matrizes simpléticas associado.

Dado um caminho $S(t)$ de matrizes simétricas definimos o operador $L_{S}: W^{1,2}\left(S^{1}=\mathbb{R} / \mathbb{Z}, \mathbb{R}^{2 n}\right) \rightarrow$ $L^{2}\left(S^{1}, \mathbb{R}^{2 n}\right)$ dado por:

$$
L_{S}(x(t))=-J \dot{x}(t)-S(t) x(t) .
$$


Estamos assumindo, para tornar a notação mais simples, que o arco é definido para $t \in[0,1]$. Notamos que $L_{S}$ é um operador auto-adjunto (para o produto interno de $L^{2}$ ) e que ele é uma perturbação compacta (pois $t$ varia no intervalo compacto $[0, T]$ ) do operador auto-adjunto $L_{0}$ dado por:

$$
L_{0}(x(t))=-J \dot{x}(t)
$$

Isso nos permite usar a Teoria da Perturbação de Kato (ver [19]) para pertubações de operadores auto-adjuntos lineares, que descreve o espectro e as autofunções de operadores deste tipo.

\subsubsection{Caso bi-dimensional}

O espectro $\sigma\left(L_{0}\right)$ do operador $L_{0}$ é o conjunto $\{2 \pi l\}$ e cada autovalor tem multiplicidade 2 . O autoespaço do autovalor $2 \pi l$ é gerado pelas funções $(\cos (2 \pi l t), \sin (2 \pi l t))$ e $(-\sin (2 \pi l t), \cos (2 \pi l t))$. É possível ordenar $\sigma\left(L_{0}\right)$ da seguinte forma: $\gamma_{k}(0)=2 \pi\left\lfloor\frac{k}{2}\right\rfloor$ (como os autovalores tem multiplicidade 2 para o operador $L_{0}$ eles aparecem 2 vezes na sequência).

Considere a deformação contínua do caminho nulo no caminho $S(t)$ dada por $s S(t)$ onde $s \in[0,1]$. A teoria da perturbação de Kato nos diz que os autovalores e autofunções de $L_{0}$ são deformados continuamente nos de $L_{s S}$, ou seja, existem funções $\widetilde{\gamma}_{k}(s S)$ e $\widetilde{\varphi}_{k}(s S)$ contínuas em $s$ dadas por:

$$
\begin{gathered}
\widetilde{\gamma}_{k}(s S) \in \sigma\left(L_{s S}\right) \text { e } \sigma\left(L_{s S}\right)=\bigcup_{k \in \mathbb{Z}}\left\{\widetilde{\gamma}_{k}(s S)\right\} \text { contando multiplicidades, } \\
\widetilde{\varphi}_{k}(s S) \text { onde } \widetilde{\varphi}_{k}(s S) \text { é uma autofunção associada a } \widetilde{\gamma}_{k}(s S) \\
\left\{\widetilde{\varphi}_{k}(s S), k \in \mathbb{Z}\right\} \text { é base de Hilbert de } L^{2}
\end{gathered}
$$

e $\widetilde{\gamma}_{k}(0)=\gamma_{k}(0)$, que é dada segundo a ordenação original de $\sigma\left(L_{0}\right)$ exibida acima.

Para o cálculo do índice de Conley-Zehdner generalizado usaremos uma ordenação monótona de $\Sigma\left(L_{S}\right)$. Essa ordenação também é obtida usando a teoria de Kato e consiste na existência de funções $\gamma_{k}(s S)$ contínuas em $s$ que satisfazem:

$$
\begin{gathered}
\gamma_{k}(s S) \leq \gamma_{k+1}(s S) \\
\sigma\left(L_{s S}\right)=\bigcup_{k \in \mathbb{Z}}\left\{\gamma_{k}(s S)\right\} \text { contando multiplicidades, }
\end{gathered}
$$

e $\gamma_{k}(0)$ é definido segundo a ordenação acima. Além disso temos que para a função $\gamma_{k}(s S)$ existem pontos $0=s_{0}<s_{1}<\ldots<s_{\beta}=1$ e inteiros $\left\{k_{0}, \ldots, k_{\beta-1}\right\}$ tais que:

para $s \in\left[s_{j}, s_{j+1}\right]$, vale $\gamma_{k}(s S)=\widetilde{\gamma}_{k_{j}}(s S)$.

Denotaremos por $\varphi_{k}(s S)$ a autofunção que associamos acima a $\gamma_{k}(s S)$ e $\left\{\varphi_{k}(s S), k \in \mathbb{Z}\right\}$ é base de Hilbert de $L^{2}$.

Feito isso, o índice de Conley-Zehnder generalizado é definido por:

$$
\widetilde{\mu}_{C Z}^{1}(S(t))=\max \left\{k \in \mathbb{Z} ; \gamma_{k}(1)<0\right\} .
$$

Nos dedicamos agora a estudar a relação entre as duas ordenações de $\sigma\left(L_{s S}\right)$ que construímos.

Nosso objetivo é mostrar que $\varphi_{k}(S)$ e $\varphi_{k}(0)=\widetilde{\varphi}_{k}(0)$ são homotópicos como laços que não passam pela origem. Esse fato será importante para provarmos algumas propriedades do índice de Conley-Zehnder generalizado. Iniciamos com o seguite lema:

Lema 1. Seja $\gamma$ um autovalor de $L_{H}$. Então se $\psi_{0}$ e $\psi_{1}$ são autofunções de $L_{H}$ associadas à $\gamma$ temos:

$\psi_{0}$ e $\psi_{1}$ são homotópicos como laços que não passam pela origem. 
Demonstração: Tomamos a seguinte homotopia $r \psi_{0}+(1-r) \psi_{1}$ entre $\psi_{0}$ e $\psi_{1}$. Temos 2 possibilidades:

a) a homotopia acima não passa pela origem e nesse caso o lema está demonstrado.

b) a homotopia acima passa pela origem.

Nesse caso existem $r^{*} \in \mathbb{R}$ e $t^{*}$ satisfazendo:

$$
r^{*} \psi_{0}\left(t^{*}\right)+\left(1-r^{*}\right) \psi_{1}\left(t^{*}\right)=0
$$

Como $\psi_{0}$ e $\frac{r^{*}-1}{r^{*}} \psi_{1}$ são soluções da equação $\dot{z}=(J s S+\gamma) z$ que se intersectam em $t^{*}$ temos pela unicidade de soluções de EDO's que se tocam em $t^{*}$, temos que $\psi_{0}=\frac{r^{*}-1}{r^{*}} \psi_{1}$. Nesse caso temos claramente $\psi_{0}$ e $\psi_{1}$ são homotópicos como laços que não passam pela origem. Isso finaliza a demonstração de nosso lema.

Observamos que isso implica que $\psi_{0}$ e $\psi_{1}$ tem o mesmo winding number.

Esse lema nos garante que sempre que ocorre a igualdade:

$$
\widetilde{\gamma}_{k}(s S)=\widetilde{\gamma}_{l}(s S),
$$

deve valer que $\widetilde{\varphi}_{k}(s S)$ e $\widetilde{\varphi}_{l}(s S)$ são homotópicos como laços que não passam pela origem. Isso implica que $\widetilde{\varphi}_{k}(0)$ e $\widetilde{\varphi}_{l}(0)$ são homotópicos como laços que não passam pela origem. Pela descrição do espectro do operador $L_{0}$ temos então que sempre vale:

$$
\left\lfloor\frac{k}{2}\right\rfloor=\left\lfloor\frac{l}{2}\right\rfloor .
$$

Estamos prontos agora, para mostrar que $\varphi_{k}(S)$ e $\varphi_{k}(0)$ são homotópicos como laços que não passam pela origem. que:

Dado $\widetilde{\gamma}_{k}(S)$ vimos que existem pontos $0=s_{0}<s_{1}<\ldots<s_{\beta}=1$ e inteiros $\left\{k_{0}, \ldots, k_{\beta-1}\right\}$ tais para $s \in\left[s_{j}, s_{j+1}\right]$, vale $\gamma_{k}(s S)=\widetilde{\gamma}_{k_{j}}(s S)$. Em particular observamos que $\widetilde{\gamma}_{k_{j}}\left(s_{j+1} S\right)=\widetilde{\gamma}_{k_{j+1}}\left(s_{j+1} S\right)$. Pelo lema anterior isso implica que $\left\lfloor\frac{k_{j+1}}{2}\right\rfloor=\left\lfloor\frac{k_{j}}{2}\right\rfloor$. Segue então que para todo $j$ ocorre $\left\lfloor\frac{k}{2}\right\rfloor=\left\lfloor\frac{k_{j}}{2}\right\rfloor$. Isso implica, por observações anteriores, que $\varphi_{k}(S)$ é homotópico como laço que não passa pela origem a $\varphi_{k}(0)$ (e portanto, tambem a $\widetilde{\varphi}_{k}(S)$ ).

No caso bi-dimensional é possível calcular $k$ usando o winding number das auto-funções de $L_{S}$. Como vimos as auto-funções $\varphi_{k}(s S)$ que estão associadas a um mesmo autovalor tem sempre o mesmo winding number, e cada inteiro inteiro $n \in \mathbb{Z}$ aparece como winding number de um par linearmente independente de autofunções de $L_{s S}$. Pelo fato de $\varphi_{k}(s S)$ ser homotópico a $\varphi_{k}(0)$ como laço que não passa pela origem, temos a seguinte monotonicidade:

$$
\operatorname{wind}\left(\varphi_{k}(s S)\right) \leq \operatorname{wind}\left(\varphi_{k+1}(s S)\right) .
$$

Definimos então $\alpha(S)=\max \left\{\operatorname{wind}\left(\varphi_{k}(S)\right) ; \gamma_{k}(S)<0\right\}$ e:

- $p(S)=1$ quando não existe autofunção $\varphi$ com winding igual a $\alpha(S)$ e associada a autovalor não-negativo,

- $p(S)=0$, caso contrário.

Proposição 4. $\widetilde{\mu}_{C Z}^{1}(S)=2 \alpha(S)+p(S)$.

Demonstração. No caso em que $S$ é o caminho nulo a descrição do auto-espaço de cada autovalor de $L_{0}$ implica a seguinte igualdade:

$$
\operatorname{wind}\left(\varphi_{k}(0)\right)=\frac{\gamma_{k}(0)}{2 \pi}=\left\lfloor\frac{k}{2}\right\rfloor .
$$

Como vimos $\varphi_{k}(0)$ e $\varphi_{k}(S)$ tem o mesmo winding number pois este é um invariante homotópico por curvas que não passam pela origem. Logo vale também wind $\left(\varphi_{k}(S)\right)=\left\lfloor\frac{k}{2}\right\rfloor$. Tomemos então 
$k_{0}=\max \left\{k ; \gamma_{k}(S)<0\right\}$. Então, por definição $\alpha(S)=\operatorname{wind}\left(\varphi_{k_{0}}(S)\right)=\left\lfloor\frac{k_{0}}{2}\right\rfloor$. Se $k_{0}$ é par vale então necessariamente a igualdade $2 \alpha(S)=k_{0}=\widetilde{\mu}_{C Z}^{1}(S)$. Mas nesse caso temos que a autofunção $\varphi_{k_{0}+1}(S)$ tem winding $\left\lfloor\frac{k_{0}+1}{2}\right\rfloor=\frac{k_{0}}{2}=\alpha(S)$ onde usamos a fórmula (2.14) e o fato de $k_{0}$ ser par. Portanto $p(S)=0$, e isso estabelece a validade da proposição quando o índice generalizado é par.

No caso em que $k_{0}$ é ímpar temos necessariamente $\alpha(S)=\operatorname{wind}\left(\varphi_{k_{0}}(S)\right)=\left\lfloor\frac{k_{0}}{2}\right\rfloor=\frac{k_{0}-1}{2}$, o que implica $2 \alpha(S)=k_{0}-1$. Mas o fato de $k_{0}$ ser ímpar implica que $\varphi_{k_{0}+1}(S)$ tem winding $\left\lfloor\frac{k_{0}+1}{2}\right\rfloor=\frac{k_{0}+1}{2}>\alpha(S)$. Como $\gamma_{k_{0}+1}(S)$ é o menor elemento não-negativo de $L_{S}$ a fórmula (2.13) então implica que não existe autovalor não-negativo com winding associado menor ou igual a $\alpha(S)$. Nessa situação temos então $p(S)=1$ e $2 \alpha(S)+p(S)=k_{0}-1+1=k_{0}=\widetilde{\mu}_{C Z}^{1}(S)$. Portanto a proposição também vale quando o índice generalizado é ímpar. Isso conclui a demonstração da proposição.

Provaremos agora que o índice generalizado e o índice geométrico coincidem para caminhos de matrizes simpléticas $2 \times 2$ não-degenerados.

Teorema 1. Se $S(t)$ é um caminho de matrizes simétricas $2 \times 2$ não-degenerado, vale $\widetilde{\mu}_{C Z}^{1}(S(t))=$ $\mu_{C Z}^{1}(S(t))$.

Demonstração. Inicialmente observamos que $\widetilde{\mu}_{C Z}$ é invariante por homotopias entre arcos não-degenerados. Isso é consequência do fato de que $\operatorname{ker}\left(L_{H}\right) \neq\{0\}$ se, e somente se, $H$ é degenerado, algo que será provado na próxima subseção. Para demonstrar a proposição precisamos provar 3 coisas:

1. $\widetilde{\mu}_{C Z}^{1}\left(e^{\pi i t}\right)=1$.

2. $\widetilde{\mu}_{C Z}^{1}(g A)=2 m^{1}(g)+\widetilde{\mu}_{C Z}^{1}(A), \forall A \in \Sigma(1)$ e $g \in G(1)$.

3. $\widetilde{\mu}_{C Z}^{1}(-A)=-\widetilde{\mu}_{C Z}^{1}(A), \forall A \in \Sigma(1)$.

-Prova de 1.

Para provar 1 observamos que o caminho $e^{\pi i t}$ está associado ao caminho de matrizes simétricas $\pi I d$. Uma análise do operador $L_{r I d}$ nos dá que seu espectro $\sigma\left(L_{r I d}\right)$ é dado por $\{r\} \cup\{(2 k) \pi-r ; k \in$ $\mathbb{Z}$ \}, sendo que os autovalores $r$ e $-r$ tem multiplicidade 1 e os demais multiplicidade 2. Pela continuidade da deformação do espectro ao longo do caminho de operadores $L_{r I d}$ para $r \in[0, \pi]$ concluí-se que $\gamma_{1}(\pi I d)=-\pi$ e $\gamma_{2}(\pi I d)=\pi$. Isso prova 1 .

-Prova de 2.

O caminho $g A$ é homotópico por caminhos não-degenerados a um caminho da forma $e^{2 \pi n i t} A$ para algum $n \in \mathbb{Z}$. Sabemos que $m^{1}\left(e^{2 \pi n i t}\right)=n$. Pela invariância homotópica do índice de Conley-Zehnder generalizado temos então $\widetilde{\mu}_{C Z}^{1}(g A)=\widetilde{\mu}_{C Z}^{1}\left(e^{2 \pi n i t} A\right)$. Basta provar então que $\widetilde{\mu}_{C Z}^{1}\left(e^{2 \pi n i t} A\right)=2 m^{1}\left(e^{2 \pi n i t}\right)+\mu_{C Z}^{1}(A)$. Para ver isso notamos que o caminho de matrizes simpléticas $B=e^{2 \pi n i t} A$ está associado ao caminho de matrizes simétricas $-J \dot{B} B^{-1}$. Um cálculo direto nos dá:

$$
-J \dot{B} B^{-1}=-J\left(e^{2 \pi n i t}\right)^{\prime} A A^{-1} e^{-2 \pi n i t}+e^{2 \pi n i t}(-J) A^{\prime} A^{-1} e^{-2 \pi n i t}=2 \pi n+e^{2 \pi n i t} H e^{-2 \pi n i t},
$$

onde $H$ é o caminho de matrizes simétricas associado a $A$. Notamos então que se $v$ é uma autofunção de $L_{H}$ associada a um autovalor $\lambda$ temos que $e^{2 \pi n i t} v(t)$ é uma autofunção de $L_{2 \pi n+H}$ associada ao mesmo autovalor $\lambda$. Entretanto, $\operatorname{wind}\left(e^{2 \pi n i t} v(t)\right)=n+\operatorname{wind}(v)$. Usando então a fórmula $\widetilde{\mu}_{C Z}^{1}\left(e^{2 \pi n i t} A\right)=2 \alpha(2 \pi n+H)+p(2 \pi n+H)$, temos igualdade dada por 2.

-Prova de 3.

Observamos que:

a) se $\widetilde{\mu}^{1}(A)$ é par, então existe $n \in \mathbb{Z}$ tal que o caminho de matrizes simpléticas $A$ é homotópico 
por caminhos não-degenerados ao caminho $B(t)=e^{2 \pi n t} \circ\left[\begin{array}{rr}e^{t} & 0 \\ 0 & e^{-t}\end{array}\right]$,

b) se $\widetilde{\mu}^{1}(A)$ é ímpar, então existe $n \in \mathbb{Z}$ tal que o caminho de matrizes simpléticas $A$ é homotópico por caminhos não-degenerados ao caminho $C(t)=e^{\pi(2 n+1) t}$.

No caso a), as propriedades 1 e 2 nos dão que $\widetilde{\mu}^{1}(B(t))=2 n$. Temos que $B^{-1}(t)=\left[\begin{array}{rr}e^{-t} & 0 \\ 0 & e^{-t}\end{array}\right]$ 。 $e^{-2 \pi n t}$, e novamente as propriedades 1 e 2 nos garantem $\widetilde{\mu}^{1}\left(B^{-1}(t)\right)=-2 n$. Pela invariância homotópica do índice generalizado isso prova a propriedade 3 no caso em $\widetilde{\mu}^{1}(A)$ é par.

No caso b) temos pelas propriedades 1 e $2 \widetilde{\mu}^{1}(C)=2 n+1$. Como $C^{-1}(t)=e^{-\pi(2 n+1) t}$ essas mesmas propriedades nos dão $\widetilde{\mu}^{1}\left(C^{-1}\right)=-(2 n+1)$. Novamente pela invariância homotópica do índice generalizado, isso prova a propriedade 3 no caso em $\widetilde{\mu}^{1}(A)$ é ímpar.

\subsubsection{Caso 2n-dimensional}

A definição do índice de Conley-Zehnder generalizado que apresentamos inicialmente é baseada em uma ordenação dos autovalores do operador $L_{S}$. No caso 2n-dimensional essa ordenação também pode ser feita. Para fazer essa ordenação fixamos uma ordenação inicial do espectro de $L_{0}$. No caso 2n-dimensional o espectro de $L_{0}$ é novamente $\sigma\left(L_{0}\right)=\{2 \pi l ; l \in \mathbb{Z}\}$ mas dessa vez cada autovalor tem multiplicidade 2 n. Assim a ordenação de $\sigma\left(L_{0}\right)$ é dada por:

$$
\begin{aligned}
& \gamma_{k}(0)=0 \text { quando } k \in\{-n+1, \ldots, 0, \ldots n\} . \\
& \gamma_{k}(0)=2 \pi l \text { quando } k \in\{2 n l-n+1, \ldots, 2 n l, \ldots, 2 n l+l\} .
\end{aligned}
$$

Usando essa ordenação como ponto de partida definimos uma ordenação para $L_{S}$ usando a Teoria da Perturbação de Kato. Essa teoria nos garante que para $s \in[0,1]$ existem funções $\gamma_{k}(s S)$ contínuas em $s$ tais que :

$$
\begin{gathered}
\gamma_{k}(s S) \in \sigma\left(L_{s S}\right) \text { e } \sigma\left(L_{s S}\right)=\bigcup_{k \in \mathbb{Z}}\left\{\gamma_{k}(s)\right\} \text { contando multiplicidades } \\
\gamma_{k}(s S) \leq \gamma_{k+1}(s)
\end{gathered}
$$

Com essa ordenação definimos o índice de Conley-Zehnder espectral pela seguinte fórmula:

$$
\widetilde{\mu}_{C Z}^{n}(S)=\max \left\{k \in \mathbb{Z} ; \gamma_{k}(S)<0\right\} .
$$

Chamamos um arco de matrizes simétricas não-degenerado (respectivamente degenerado) se seu caminho associado de matrizes simpléticas é não-degenerado (respectivamente degenerado). Provaremos agora a seguinte proposição:

Proposição 5. Se $S(t), t \in[0,1]$, é um caminho de matrizes simétricas não-degenerado, vale a igualdade:

$$
\widetilde{\mu}_{C Z}^{n}(S)=\mu_{C Z}^{n}(S) .
$$

Demonstração: Iremos assumir nessa demonstração a validade do teorema para o caso bidimensional e estabelecer essa igualdade para $n>1$.

Passo 1: Nesse passo provamos a invariância homotópica do índice generalizado.

Dizemos que 2 arcos não-degenerados de matrizes simétricas são homotópicos quando seus arcos associados de matrizes simpléticas são homotópicos por arcos em $\Sigma(n)$. Suponha que $S S^{\prime}$ são arcos homotópicos não-degenerados de matrizes simétricas. Considere $C_{s}$ uma homotopia por caminhos não-degenerados entre $S$ e $S^{\prime}$ com $C_{0}=S$ e $C_{1}=S^{\prime}$. Então para todo $s \in[0,1]$ vale $\operatorname{ker}\left(L_{C_{s}}\right)=\{0\}$. Isso é decorrente da seguinte afirmação:

Afirmação. $\operatorname{ker}\left(L_{H}\right) \neq\{0\}$ se, e somente se, $H(t)$ é um arco degenerado de matrizes simétricas. 
Prova da afirmação:

Suponha $\operatorname{ker}\left(L_{H}\right) \neq\{0\}$ e seja $x(t) \neq 0 \in \operatorname{ker}\left(L_{H}\right)$. Seja $A(t)$ o caminho de matrizes simpléticas associado à $H(t)$. Então $A(t)$ é a única solução do problema de valor inicial:

$$
\left\{\begin{aligned}
\dot{A}(t) & =J H(t) A(t) \\
A(0) & =I d
\end{aligned}\right.
$$

Analogamente $x(t)$ é a única solução da EDO:

$$
\dot{x}(t)=J H(t) x(t) .
$$

com condição inicial $x(0)$.

Mas o caminho $y(t)=A(t) x(0)$ também satisfaz:

$$
\begin{gathered}
\dot{y}(t)=A(t) \dot{x}(0)=\dot{A}(t) x(0)=J H(t) A(t) x(0)=J H(t) y(t) \\
y(0)=A(0) x(0)=x(0)
\end{gathered}
$$

logo $y(t)=x(t)$. Observamos que $x(0) \neq 0$ pois caso isso ocorra, decorre da unicidade de soluções para EDOs que $x(t)$ é o caminho nulo. Como $x(t) \in \operatorname{ker}\left(L_{H}\right), x(t)$ é um caminho fechado e $x(0)=x(1)$. Assim

$$
A(1) x(0)=y(1)=x(1)=x(0),
$$

e, portanto, $x(0)$ é um autovetor de $A(1)$ associado ao autovalor 1 . Logo $A(t)$ é um caminho degenerado, e isso prova uma das direções da afirmação.

Para provar a recíproca, suponha que $A(t)$ seja um caminho degenerado. Seja $v$ um autovetor de $A(1)$ associado ao autovalor 1 . Tome $x(t)=A(t) v . x(1)=A(1) v=v=x(0)$, logo $x(t)$ é um caminho fechado de $[0,1]$ em $\mathbb{R}^{2 n}$. Além disso $x(t)$ satisfaz a seguinte equação diferencial:

$$
\dot{x}(t)=A \dot{(t)} v=\dot{A}(t) v=J H(t) A(t) v=J H(t) x(t),
$$

$\operatorname{logo}, x(t) \in \operatorname{ker}\left(L_{H}\right), \mathrm{e} \operatorname{ker}\left(L_{H}\right) \neq\{0\}$. Isso conclui a demonstração da afirmação.

Continuando a demonstração do Passo $1, \operatorname{ker}\left(L_{C_{s}}\right)=\{0\}$ para todo $s \in[0,1]$. Usando a homotopia $C_{s}$, a Teoria da Perturbação de Kato garante a deformação contínua do espectro de $L_{S}$ no espectro de $L_{S^{\prime}}$. Em outras as palavras as funções $\gamma_{k}\left(C_{s}\right)$ construídas acima para ordenar o espectro de $L_{C_{s}}$ são contínuas em $s$. Isso garante que vale:

$$
\gamma_{k}(S)<0 \Leftrightarrow \gamma_{k}\left(S^{\prime}\right)<0
$$

Para demonstrar isso notamos que caso ocorresse $\gamma_{k}(S)<0$ e $\gamma_{k}\left(S^{\prime}\right) \geq 0$, teríamos por continuidade $\gamma_{k}\left(C_{s}\right)=0$ para algum $s \in[0,1]$. Mas isso contradiz $\operatorname{ker}\left(L_{C_{s}}\right)=\{0\}$, logo não pode ocorrer. Assim estabelecemos a ida da equação (2.27). A volta é uma repetição do mesmo argumento trocando $S$ por $S^{\prime}$.

Segue então da equação $(2.27)$ que :

$\max \left\{k ; \gamma_{k}(S)<0\right\}=\max \left\{k ; \gamma_{k}\left(S^{\prime}\right)<0\right\}$ o que implica $\widetilde{\mu}_{C Z}^{n}(S)=\widetilde{\mu}_{C Z}^{n}\left(S^{\prime}\right)$.

Fim do Passo 1.

Passo 2: Nesse passo provamos a coincidência do índice de Conley-Zehnder generalizado e do índice de Conley-Zehnder. Tomemos então $S(t)$ para $t \in[0,1]$ um caminho de matrizes simétricas não-degenerado. Sabemos então que $S$ é homotópico por caminhos não degenerados a um caminho 
da forma $U(t) \oplus H_{\tau} \oplus \ldots \oplus H_{\tau}$ ( $H_{\tau}$ é somada $n-1$ vezes) onde $U(t)$ é um caminho de matrizes simétricas $2 \times 2$ e $H_{\tau}$ é dada por:

$$
H_{\tau}=\left[\begin{array}{cc}
0 & \tau \\
\tau & 0
\end{array}\right]
$$

A existência dessa homotopia decorre do fato de que o índice de Conley-Zehnder classifica totalmente os elementos de $\Sigma(n)$ a menos de homotopia. A invariância homotópica do índice generalizado e do índice axiomático garantem que:

$$
\begin{aligned}
& \widetilde{\mu}_{C Z}^{n}(S)=\widetilde{\mu}_{C Z}^{n}\left(U(t) \oplus H_{\tau} \oplus \ldots \oplus H_{\tau}\right) \\
& \mu_{C Z}^{n}(S)=\mu_{C Z}^{n}\left(U(t) \oplus H_{\tau} \oplus \ldots \oplus H_{\tau}\right)
\end{aligned}
$$

Como $U(t)$ é um caminho de matrizes simétricas bidimensional temos ainda:

$$
\widetilde{\mu}_{C Z}^{1}(U)=\mu_{C Z}^{1}(U)=\mu_{C Z}^{n}\left(U(t) \oplus H_{\tau} \oplus \ldots \oplus H_{\tau}\right)=\mu_{C Z}^{n}(S)
$$

A segunda igualdade de (2.31) é decorrente do fato de que as matrizes simétricas $H_{\tau}$ não dão nenhuma contribuição ao índice de Conley-Zehnder de $U(t) \oplus H_{\tau} \oplus \ldots \oplus H_{\tau}$, um fato que será demonstrado em uma observação abaixo na qual estudaremos esse caminho detalhadamente. Combinando (2.29) e (2.31) notamos que para finalizar a demonstração de nossa proposição basta provar que:

$$
\widetilde{\mu}_{C Z}^{1}(U)=\widetilde{\mu}_{C Z}^{n}\left(U(t) \oplus H_{\tau} \oplus \ldots \oplus H_{\tau}\right) .
$$

Demonstração de 2.32:

Seja $k_{0}=\widetilde{\mu}_{C Z}^{1}(U)$. Então pela continuidade das funções $\gamma_{k}(s U)$ que dão a ordenação do espectro de $L_{s U}$, existe $R>0$ tal que:

$$
\gamma_{k}(s U) \in(-R, R) \forall k \in\left\{-\left|k_{0}\right|-7, \ldots, 0, \ldots,\left|k_{0}\right|+7\right\} \text { e } s \in[0,1] .
$$

Observamos que $\sigma\left(L_{H_{\tau} \oplus \ldots \oplus H_{\tau}}\right)=\left\{ \pm \sqrt{\tau^{2}+(2 \pi k)^{2}} ; k \in \mathbb{Z}\right\}$, onde cada autovalor tem multiplicidade $2 n-2$ exceto $\tau$ e $-\tau$ que tem multiplicidade $n-1$. Logo, se tomarmos $\tau_{0}$ com $\left|\tau_{0}\right|>R$, temos que, todos os autovalores de $L_{U(t) \oplus H_{\tau} \oplus \ldots \oplus H_{\tau}}$ que estão no intervalo $(-R, R)$ são autovalores de $L_{s U}$. Segue disso que para $k \in\left\{-\left|k_{0}\right|-7, \ldots, 0, \ldots,\left|k_{0}\right|+7\right\}$, existe $m_{0}$ tal que:

$$
\gamma_{k}(s U)=\gamma_{m_{0}+k}\left(s U \oplus H_{\tau} \oplus \ldots \oplus H_{\tau}\right), \forall s \in[0,1] .
$$

Nosso objetivo agora é mostrar que $m_{0}=0$ para $s=0$. Considere o operador $L_{0 \oplus H_{\tau} \oplus \ldots \oplus H_{\tau}}$. Se variarmos $\tau$ entre 0 e $\tau_{0}$ teremos um caminho deformando $L_{0}$ em $L_{0 \oplus H_{\tau} \oplus \ldots \oplus H_{\tau}}$, que pela teoria da perturbação de Kato nos permitirá ordenar os autovalores de $L_{0 \oplus H_{\tau} \oplus \ldots \oplus H_{\tau}}$. Quando $\tau=0$ os autovalores são ordenados com:

$$
\gamma_{k}(0)=0 \forall k \in\{-n+1, \ldots, 0, . . n\}
$$

Para $\tau^{\prime}<2 \pi$ pequeno temos os autovalores $-\tau$ e $\tau$ tem multiplicidade $n-1$ para o operador

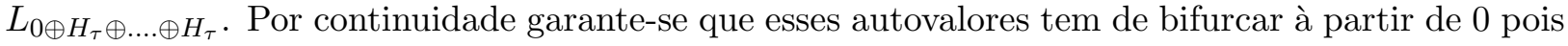
os números entre $\tau^{\prime}$ e $2 \pi$ não são autovalores para nenhum operador $L_{0 \oplus H_{\tau} \oplus \ldots . \oplus H_{\tau}}$ quando $\tau$ está em $\left[0, \tau^{\prime}\right]$. A monotonicidade então força que a única ordenação possível seja:

$$
\left\{\begin{array}{l}
\gamma_{k}\left(0 \oplus H_{\tau^{\prime}} \oplus \ldots \oplus H_{\tau^{\prime}}\right)=-\tau^{\prime} \text { se } k \in\{-n+1, . .,-1\} \\
\gamma_{k}\left(0 \oplus H_{\tau^{\prime}} \oplus \ldots \oplus \oplus H_{\tau^{\prime}}\right)=0 \text { se } k \in\{0,1\} \\
\gamma_{k}\left(0 \oplus H_{\tau^{\prime}} \oplus \ldots \oplus H_{\tau^{\prime}}\right)=\tau \text { se } k \in\{2, . ., n\}
\end{array}\right.
$$

Agora aumentamos $\tau$ de $\tau^{\prime}$ até $\tau_{0}$. Ao fazermos isso nunca aparecem autovalores no intervalo $\left[-\tau^{\prime}, \tau^{\prime}\right]$, excetuando o 0 que tem multiplicidade 2. Assim, a única escolha possível para deformar 
um autovalor de $L_{0 \oplus H_{\tau^{\prime}} \oplus \ldots \oplus H_{\tau^{\prime}}}$ no autovalor 0 de $L_{0 \oplus H_{\tau_{0}} \oplus \ldots \oplus H_{\tau_{0}}}$ é fazer $\gamma_{0}\left(0 \oplus H_{\tau} \oplus \ldots \oplus H_{\tau}\right)=$ $\gamma_{1}\left(0 \oplus H_{\tau} \oplus \ldots \oplus H_{\tau}\right)=0$ para todo $\tau \in\left[\tau^{\prime}, \tau_{0}\right]$.

Isso garante que $m_{0}=0$ pois:

$$
\gamma_{0}(0)=\gamma_{1}(0)=0=\gamma_{0}\left(0 \oplus H_{\tau} \oplus \ldots \oplus H_{\tau}\right)=\gamma_{1}\left(0 \oplus H_{\tau} \oplus \ldots \oplus H_{\tau}\right),
$$

e o autovalor 0 tem multiplicidade 2 para os dois operadores em questão. Isso elimina a possibilidade de haver uma igualdade entre $\gamma_{0}(0)$ e $\gamma_{1}(0)$ com qualquer outro autovalor de $L_{0 \oplus H_{\tau_{0}} \oplus \ldots \oplus H_{\tau_{0}}}$ que não seja $\gamma_{0}\left(0 \oplus H_{\tau_{0}} \oplus \ldots \oplus H_{\tau_{0}}\right)$ ou $\gamma_{1}\left(0 \oplus H_{\tau_{0}} \oplus \ldots \oplus H_{\tau_{0}}\right)$. Se igualdade $(2.34)$ valesse para $m_{0} \neq 0$ deveria haver uma igualdade entre $\gamma_{0}(0)$ ou $\gamma_{1}(0)$ e um autovalor que não fosse $\gamma_{0}\left(0 \oplus H_{\tau_{0}} \oplus \ldots \oplus H_{\tau_{0}}\right)$ ou $\gamma_{1}\left(0 \oplus H_{\tau_{0}} \oplus \ldots \oplus H_{\tau_{0}}\right)$, o que causaria um absurdo.

Usando que $m_{0}=0$ garante-se que:

$$
\gamma_{k}(U)=\gamma_{k}\left(U \oplus H_{\tau} \oplus \ldots \oplus H_{\tau}\right) \forall s \in[0,1] \forall k \in\left\{-\left|k_{0}\right|-7, \ldots,\left|k_{0}\right|+7\right\} .
$$

Assim a ordenação dos autovalores de $L_{U}$ e $L_{U \oplus H_{\tau} \oplus \ldots \oplus H_{\tau}}$ coincide justamente na parte do espectro de $L_{U}$ onde ocorre a transição de autovalores negativos para autovalores positivos que é o conjunto $\left\{\gamma_{k}(U) ; k \in\left\{-\left|k_{0}\right|-7, \ldots,\left|k_{0}\right|+7\right\}\right\}$. Logo essa transição de autovalores negativos para positivos ocorre simultaneamente nos dois operadores o que implica a igualdade:

$$
\max \left\{k ; \gamma_{k}(U)<0\right\}=\max \left\{k ; \gamma_{k}\left(U \oplus H_{\tau_{0}} \oplus \ldots \oplus H_{\tau_{0}}\right)<0\right\},
$$

o que é equivalente a $\widetilde{\mu}_{C Z}^{1}(U)=\widetilde{\mu}_{C Z}^{n}\left(U \oplus H_{\tau} \oplus \ldots \oplus H_{\tau}\right)$ e portanto finaliza a demonstração da igualdade (2.32), e consequentemente da proposição.

\section{Estudo do operador $L_{H_{\tau}}$}

Inicialmente estudamos o espectro desse operador. $\lambda$ é um autovalor, se e somente se, existe uma função $x(t)$ de $\mathbb{R}$ em $\mathbb{R}^{2}$ periódica de período 1 que satisfaça a seguinte equação diferencial

$$
\dot{x}=\left[\begin{array}{rr}
-\tau & -\lambda \\
\lambda & \tau
\end{array}\right] x
$$

A existência de uma solução com essas propriedades é, portanto, equivalente à existência de soluções periódicas de período 1 para a EDO linear acima. A existência de soluções periódicas só pode ocorrer quando os autovalores da matriz $\left[\begin{array}{rr}-\tau & -\lambda \\ \lambda & \tau\end{array}\right]$ forem nulos ou imaginários puros.

Os autovalores de $\left[\begin{array}{rr}-\tau & -\lambda \\ \lambda & \tau\end{array}\right]$ são as soluções da seguinte equação:

$$
p^{ \pm}= \pm \sqrt{\tau^{2}-\lambda^{2}}
$$

e 0 é autovalor de $\left[\begin{array}{rr}-\tau & -\lambda \\ \lambda & \tau\end{array}\right]$ apenas para $\lambda= \pm \tau$. Nesse caso os autoespaços associados à $\tau$ e $-\tau$ tem dimensão 1 , o que decorre do fato de que substituindo $\lambda$ por um desses valores, não torna a matriz do sistema (2.38) nula.

Os autovalores $p^{ \pm}$são imaginários puros apenas quando $|\lambda|>|\tau|$, nesse caso eles são $p^{ \pm}=$ $\pm i \sqrt{\lambda^{2}-\tau^{2}}$. Nesse caso a teoria de EDO nos diz que as órbitas são sempre fechadas e todas tem o mesmo período mínimo que é $\frac{2 \pi}{\sqrt{\lambda^{2}-\tau^{2}}}$. Assim 1 é período de órbitas desse fluxo se, e somente se, existe $k \in \mathbb{Z}$ tal que $k \frac{2 \pi}{\sqrt{\lambda^{2}-\tau^{2}}}=1$, o que é equivalente à $\lambda^{2}=(2 k \pi)^{2}+\tau^{2}$ ou seja:

$$
\lambda=\sqrt{(2 k \pi)^{2}+\tau^{2}},
$$

para algum $k \in \mathbb{Z}$. Cada um desses autovalores tem multiplicidade 2, pois nesse caso todas as soluções do sistema (2.38) são fechadas para $t=1$ e esse conjunto de soluções é bidimensional. Conseguimos portanto descrever completamente o espectro de $L_{H_{\tau}}$. No caso do operador 
$L_{H_{\tau} \oplus \ldots \oplus H_{\tau}}$ (soma direta $n-1$ vezes) os autovalores continuam sendo os mesmos mas a multiplicidade destes é multiplicada por $n-1$. Essa análise é feita exatamente como a acima, mas o sistema linear associado tem dimensão $2(n-1)$.

Usaremos na seção seguinte uma importante propriedade do índice de Conley-Zehnder enunciada em [17], a dizer que quando $E(t)$ é um caminho de matrizes simétricas positivas definidas $2 n \times 2 n$ vale:

$$
\gamma_{k}(E)<0 ; k \in\{-n+1, \ldots, 0,1, \ldots, n\} .
$$

Exibimos agora, a prova desta propriedade no caso bi-dimensional. Para a demonstração no caso geral (que usa uma caracterização geométrica do índice de Conley-Zehnder no caso $2 n$-dimensional) referimos a [20] e [3].

Prova da propriedade no caso bi-dimensional:

Como provamos anteriormente $\varphi_{k}(0)$ e $\varphi_{k}(E)$ são homotópicos como laços que não passam pela origem. Como o winding number é invariante por homotopias deste tipo, temos que o $\operatorname{wind}\left(\varphi_{k}(0)\right)=\operatorname{wind}\left(\varphi_{k}(E)\right)$. Essa igualdade implica:

$$
\operatorname{wind}\left(\varphi_{0}(E)\right)=\operatorname{wind}\left(\varphi_{1}(E)\right)=0 .
$$

É possível calcular a derivada do argumento de $\varphi_{k}(E)$ pela seguinte fórmula:

$\operatorname{ar} g\left(\varphi_{k}(E)\right)=\frac{\left\langle\dot{\varphi}_{k}(E), J \varphi_{k}(E)\right\rangle}{\left\langle\varphi_{k}(E), \varphi_{k}(E)\right\rangle}=\frac{\left\langle J E \varphi_{k}(E)+\gamma_{k}(E) J \varphi_{k}(E), J \varphi_{k}(E)\right\rangle}{\left\langle\varphi_{k}(E), \varphi_{k}(E)\right\rangle}=\frac{\left\langle E \varphi_{k}(E), \varphi_{k}(E)\right\rangle}{\left\langle\varphi_{k}(E), \varphi_{k}(E)\right\rangle}+\gamma_{k}(E)$,

onde foi usado que $\dot{\varphi}_{k}(E)=J E \varphi_{k}(E)+\gamma_{k}(E) J \varphi_{k}(E)$.

Observamos que o termo $\frac{\left\langle E \varphi_{k}(E), \varphi_{k}(E)\right\rangle}{\left\langle\varphi_{k}(E), \varphi_{k}(E)\right\rangle}$ independe da norma de $\varphi_{k}(E)$, depende apenas de sua direção.

Lembramos agora que o winding number de $\varphi_{k}(E)$ pode ser calculado segundo a integral de $\arg \left(\varphi_{k}(E)\right)$, ou seja:

$$
\operatorname{wind}\left(\varphi_{k}(E)\right)=\int_{S^{1}} \arg \left(\varphi_{k}(E)\right)
$$

Usando as duas fórmulas acima para $l=0,1$ obtemos:

$$
0=\operatorname{wind}\left(\varphi_{l}(E)\right)=\int_{S^{1}} \arg \left(\varphi_{l}(E)\right)=\int_{S^{1}} \frac{\left\langle E \varphi_{l}(E), \varphi_{l}(E)\right\rangle}{\left\langle\varphi_{l}(E), \varphi_{l}(E)\right\rangle}+\gamma_{l}(E)
$$

Do fato de $E$ ser positiva definida e $\varphi_{l}(E)$ ser uma curva não-nula, temos $\gamma_{l}(E)=-\int_{S^{1}} \frac{\left\langle E \varphi_{l}(E), \varphi_{l}(E)\right\rangle}{\left\langle\varphi_{l}(E), \varphi_{l}(E)\right\rangle}<$ 0 o que prova a propriedade no caso bidimensional.

\subsection{Estimativa para o índice de Conley-Zehnder em hipersuperfícies convexas}

Nesta seção provaremos o mais importante resultado deste capítulo que é uma estimativa do índice de Conley-Zehnder de órbitas periódicas em hipersuperfícies convexas de $\mathbb{R}^{4}$. Essa estimativa será importante para a análise de bubbling feita no capítulo 3, pois fornecerá um controle adicional para o surgimento dos pontos de bubbling.

Teorema 2. Seja $S \subset \mathbb{R}^{4}$ uma hipersuperfície estritamente convexa em relação à origem. Então para toda órbita periódica $P$ do fluxo de Reeb associado à $S$ (que definimos no Capítulo 1) temos $\widetilde{\mu}_{C Z}^{1}(P) \geq 3$. 
Comentário:

Segue diretamente do teorema que no caso de a órbita ser um recobrimento não-trivial de uma órbita periódica, a estimativa mínima é 5 .

Demonstração do teorema: Definimos inicialmente um Hamiltoniano $H$ tal que $H^{-1}(1)=S$ e $H(t x)=t^{2} H(x)$. Essa pode ser substituída pela hipótese mais fraca de que o nível $S$ é regular. Apenas a colocamos aqui pois nesse caso a dinâmica restrita a $S$ é a do fluxo de Reeb, sem ser necessário fazer uma reparametrização (como foi observado no capítulo 1).

Seja $P$ uma órbita periódica de período $T$. A matriz do fluxo linearizado $A(t)=D \phi^{t}(P(t))$ satisfaz a seguinte equação diferencial:

$$
\dot{A}(t)=J H^{\prime \prime}(P(t)) A(t)
$$

onde $H^{\prime \prime}$ é a matriz Hessiana de $H$.

Como o nível $S$ é estritamente convexo, existe $\epsilon>0$ tal que:

$$
H^{\prime \prime}(x)>\epsilon I d, \forall x \in S
$$

Pela observação acima temos que o índice de Conley-Zehnder da órbita $P$ é necessariamente maior ou igual a 2, pois pelo fato de $H^{\prime \prime}$ ser positiva-definida temos que:

$$
\gamma_{k}\left(L_{s I d}\right)=-s \forall k \in\{-1,0,1,2\}
$$

Isso prova a estimativa índice de Conley-Zehnder $\tilde{\mu}_{C Z}^{2}(P) \geq 2$.

Iremos agora construir uma trivialização global e simplética de $T_{S} \mathbb{R}^{4}$. Essa trivialização é construída da seguinte forma. Seja $\nabla H$ o campo o gradiente da função Hamiltoniana. Então usando os quatérnions $i, j, k$ é possível gerar a seguinte trivialização de $T_{S} \mathbb{R}^{4}:\left\{\frac{\nabla H}{|\nabla H|}, i \frac{\nabla H}{|\nabla H|}, j \frac{\nabla H}{|\nabla H|}, k \frac{\nabla H}{|\nabla H|}\right\}$. Afirmamos que essa trivialização é simplética. Como $\{(1,0,0,0), i, j, k\}$ é uma base simplética de $\left(\mathbb{R}^{4}, \omega_{0}\right)$, e que a multiplicação à esquerda por um quatérnion de norma 1 é um simplectomorfismo.

Essa trivialização é homotópica à trivialização dada por $\left\{\frac{x}{|x|}, i \frac{x}{|x|}, j \frac{x}{|x|}, k \frac{x}{|x|}\right\}$ pois como o nível $S$ é convexo, os campos $x$ e $\nabla H(x)$ são isotópicos como campos transversais a $S$. Isso implica que ao calcular o índice de Conley-Zehnder nessas duas trivialização o resultado será o mesmo. Observamos que o fluxo Hamiltoniano $\phi^{t}$ preserva os campos $\nabla H$ e $i \nabla H=J_{0} \nabla H=X_{H}$ (onde $J_{0}$ é a estrutura complexa canônica de $\left.\mathbb{R}^{4}\right)$. Ele preserva, portanto, também os campos $\frac{\nabla H}{|\nabla H|}$ e $i \frac{\nabla H}{|\nabla H|}$ e a distribuição de planos $\eta$ gerada por $\left.\left\{\frac{\nabla H}{|\nabla H|}, i \frac{\nabla H}{|\nabla H|}\right\}\right\}$. O ortogonal simplético de $\eta$ é justamente o conjunto de planos $\xi$ gerado por $\left\{j \frac{\nabla H}{|\nabla H|}, k \frac{\nabla H}{|\nabla H|}\right\}$ (chamamos essa distribuição de $\xi$ porque ela é a estrutura de contato quando pensamos em $S$ como um nível de energia de contato).

Com relação a essa nova trivialização que construímos, o caminho de matrizes simpléticas que descreve o fluxo linearizado sobre $P$ é da forma $0 \oplus B(t)$. Associado a ele temos um caminho de matrizes simétricas $0 \oplus S(t)$. O índice de Conley-Zehnder que desejamos calcular é o relativo ao campo de Reeb que é dado por $\widetilde{\mu}_{C Z}^{1}(S(t))$.

Como os caminhos de matrizes simétricas $H^{\prime \prime}(P(t))$ e $0 \oplus S(t)$ geram o mesmo fluxo linearizado sobre $P$, apenas usando trivializações diferentes para descrevê-lo, temos a seguinte igualdade:

$$
\operatorname{dim} \operatorname{ker}\left(L_{H^{\prime \prime}(P(t))}\right)=\operatorname{dim} \operatorname{ker}\left(L_{0 \oplus S(t)}\right)=\operatorname{dim} \operatorname{ker}\left(L_{S(t)}\right)+2
$$

Suponhamos inicialmente que o caminho $B(t)$ é não-degenerado, nesse caso $\operatorname{dim} \operatorname{ker}\left(L_{S(t)}\right)=0$ e $\operatorname{dim} \operatorname{ker} L_{H^{\prime \prime}(P(t))}=2$ Para calcular $\widetilde{\mu}_{C Z}^{1}(P)=\widetilde{\mu}_{C Z}^{1}(S(t))$ temos a seguinte estimativa:

$$
\begin{gathered}
\widetilde{\mu}_{C Z}^{1}(S(t))=\widetilde{\mu}_{C Z}^{2}\left(H_{\tau} \oplus S(t)\right)=\widetilde{\mu}_{C Z}^{2}(0 \oplus S(t))+1= \\
=\max \left\{k \in \mathbb{Z} ; \gamma_{k}(0 \oplus S(t)) \leq 0\right\}-1=\max \left\{k \in \mathbb{Z}, \gamma_{k}\left(H^{\prime \prime}(P(t)) \leq 0\right\}-1\right.
\end{gathered}
$$


Devido à importância dessa estimativa explicaremos o que usamos em cada igualdade da fórmula acima:

1) a primeira igualdade segue da propriedade do índice de Conley-Zehnder que nos garante $\widetilde{\mu}_{C Z}^{2}\left(H_{\tau} \oplus S(t)\right)=\widetilde{\mu}_{C Z}^{1}\left(H_{\tau}\right)+\widetilde{\mu}_{C Z}^{1}(S(t))=0+\widetilde{\mu}_{C Z}^{1}(S(t))$

2) na segunda igualdade usamos que quando deformamos $H_{\tau}$ em 0 um dos autovalores negativos de $H_{\tau}$ colapsa no autovalor nulo. Por isso devemos somar 1 no lado esquerdo, para compensar esse autovalor negativo que foi "perdido"

3) na terceira igualdade usamos que o autovalor 0 tem multiplicidade 2 para o operador $L_{0 \oplus S(t)}$, e que, portanto $\max \left\{k \in \mathbb{Z} ; \gamma_{k}(0 \oplus S(t)) \leq 0\right\}=\max \left\{k \in \mathbb{Z} ; \gamma_{k}(0 \oplus S(t))<0\right\}+2$.

4) a quarta igualdade utiliza apenas a equivalência entre os operadores $L_{0 \oplus S(t)}$ e $L_{H^{\prime \prime}(P(t))}$.

Prosseguindo agora temos a seguinte estimativa para $\max \left\{k \in \mathbb{Z}, \gamma_{k}\left(H^{\prime \prime}(P(t)) \leq 0\right\}\right.$ :

$$
\max \left\{k \in \mathbb{Z}, \gamma_{k}\left(H^{\prime \prime}(P(t)) \leq 0\right\}=\widetilde{\mu}_{C Z}^{2}\left(H^{\prime \prime}(P(t))\right)+\operatorname{dim} \operatorname{ker} L_{H^{\prime \prime}(P(t))} \geq 2+2=4\right.
$$

Ao combinarmos as fórmulas (2.46) e (2.47) temos:

$$
\begin{gathered}
\tilde{\mu}_{C Z}^{1}\left((S(t))=\max \left\{k \in \mathbb{Z}, \gamma_{k}\left(H^{\prime \prime}(P(t)) \leq 0\right\}-1=\right.\right. \\
=\widetilde{\mu}_{C Z}^{2}\left(H^{\prime \prime}(P(t))\right)+\operatorname{dim} \operatorname{ker} L_{H^{\prime \prime}(P(t))}-1 \geq 2+2-1=3
\end{gathered}
$$

e concluímos a demonstração do teorema no caso não-degenerado.

Caso degenerado

Para esse caso inicialmente perturbamos o caminho $B(t)$ a um caminho $\tilde{B}(t)$ não degenerado, com o mesmo índice de Conley-Zehnder.

$$
\begin{array}{r}
\widetilde{\mu}_{C Z}^{1}\left((B(t))=\widetilde{\mu}_{C Z}^{1}\left((\tilde{B}(t))=\mu_{C Z}^{1}\left((\tilde{B}(t))=\widetilde{\mu}_{C Z}^{2}\left(\left(H_{\tau} \oplus \tilde{B}(t)\right)=\right.\right.\right.\right. \\
=\widetilde{\mu}_{C Z}^{2}\left((0 \oplus \tilde{B}(t))+1=\max \left\{k \in \mathbb{Z} ; \gamma_{k}(0 \oplus \tilde{B}(t)) \leq 0\right\}-1=\right. \\
=\max \left\{k \in \mathbb{Z} ; \gamma_{k}(0 \oplus B(t)) \leq 0\right\}-1-\operatorname{dim} \operatorname{ker}\left(L_{S}\right)= \\
=\max \left\{k \in \mathbb{Z} ; \gamma_{k}\left(H^{\prime \prime}(P(t))\right) \leq 0\right\}-1-\operatorname{dim} \operatorname{ker}\left(L_{S}\right)
\end{array}
$$

No caso degenerado a fórmula (2.47) é substituída por:

$$
\begin{gathered}
\max \left\{k \in \mathbb{Z}, \gamma_{k}\left(H^{\prime \prime}(P(t)) \leq 0\right\}=\widetilde{\mu}_{C Z}^{2}\left(H^{\prime \prime}(P(t))\right)+\operatorname{dim} \operatorname{ker} L_{H^{\prime \prime}(P(t))} \geq\right. \\
\geq 2+2+\operatorname{dim} \operatorname{ker}\left(L_{S}\right)=4+\operatorname{dim} \operatorname{ker}\left(L_{S}\right)
\end{gathered}
$$

Combinando as duas últimas fórmulas obtemos:

$$
\begin{aligned}
\tilde{\mu}_{C Z}^{1}((B(t))= & \max \left\{k \in \mathbb{Z} ; \gamma_{k}\left(H^{\prime \prime}(P(t))\right) \leq 0\right\}-1-\operatorname{dim} \operatorname{ker}\left(L_{S}\right) \geq \\
& \geq 4+\operatorname{dim} \operatorname{ker}\left(L_{S}\right)-1-\operatorname{dim} \operatorname{ker}\left(L_{S}\right)=3
\end{aligned}
$$

Isso finaliza a demonstração do teorema.

Como veremos adiante a propriedade de ter todas as órbitas periódicas com índice de ConleyZehnder maior ou igual a 3 tem importantes consequências dinâmicas. Isso e o fato de que a propriedade de um nível de energia de contato ser convexo não ser uma propriedade invariante por simplectomorfismos de $\mathbb{R}^{4}$ levou à seguinte definição, feita em [17]:

Definição 1. Dizemos que uma forma de contato $\lambda$ em $S^{3}$ é dinamicamente convexa quando todas órbitas periódicas do fluxo de $X_{\lambda}$ tem índice de Conley-Zehnder maior ou igual a 3. 
Além de níveis de energia convexos um outro importante exemplo de formas dinamicamente convexas foi exposto em [9]:

-se $\lambda$ é a forma de contato tight em $S^{3}$ associada ao fluxo geodésico de uma métrica de curvatura $K$ com $1 \leq K<4$, então $\lambda$ é dinamicamente convexa. 


\section{Capítulo 3}

\section{Construção do plano de energia finita para formas de contato tight em $S^{3}$}

Neste capítulo iremos expor a construção de um plano de energia finita para a simplectização de um nível de energia estritatemente convexo $M$ mergulhado em $\mathbb{R}^{4}$, devida à Hofer, Wysocki e Zehnder. Essa construção é baseada em um resultado de existência de um plano de energia finita para o caso especial no qual o nível de energia convexo é um elipsóide irracional $\mathrm{E}$ em $\mathbb{R}^{4}$, e um argumento de arraste desse plano de energia finita do elipsóide para o convexo. Um ingrediente principal para essa construção é um argumento de compacidade para certas famílias de planos de energia finita no cobordismo simplético entre E e $M$ (que será definido abaixo). Esse argumento que usa a chamada análise de bubbling será exposto abaixo com detalhes.

\subsection{Definições}

Seja $M$ uma nível de energia convexo para um Hamiltoniano $\mathrm{H}$, onde $\mathrm{H}$ satisfaz as condições: $H^{-1}(1)=M$ e $H(t x)=t^{2} H(x)$. Como vimos no capítulo 1 o fluxo Hamiltoniano nesse nível de energia é igual ao fluxo de Reeb para uma forma de contato $\lambda$ em $S^{3}\left(\lambda(p)=\frac{1}{(H(p))^{2}} \lambda_{0}(p)\right.$ onde $\lambda_{0}$ é a forma de contato tight canônica em $S^{3}$ ). Seja $\mathrm{E}$ um elipsóide irracional em $\mathbb{R}^{4}$ suficientemente grande para conter o nível de energia $M$, e considere $\lambda_{E}$ a forma de contato induzida por E em $S^{3}$. Usando coordenadas complexas $\left(z_{1}, z_{2}\right)$ em $\mathbb{R}^{4}$, temos $\lambda_{E}=f_{E} \lambda_{0}$ para $f_{E}(p)=\left(\frac{a^{2} b^{2}}{a^{2}\left|z_{1}\right|^{2}+b^{2}\left|z_{2}\right|^{2}}\right)^{2}$, onde $\frac{a^{2}}{b^{2}}$ é irracional. Para o elipsóide suficientemente grande escolhido temos $\frac{1}{H(p)}<f_{E}(p), \quad \forall p \in S^{3}$.

Definimos então uma função $h: \mathbb{R} \times S^{3} \rightarrow \mathbb{R}$ com as seguintes propriedades:

$$
\begin{aligned}
& h(a, p)=f_{E}(p) \text { se } a \geq 2, \\
& h(a, p)=f(p) \text { se } a \leq-2, \\
& \frac{\partial h}{\partial a} \geq 0, \\
& \exists \delta>0 \text { tal que } \frac{\partial h}{\partial a}(p, a)>\delta \quad \forall a \in[-1,1] .
\end{aligned}
$$

Consideramos então em $\mathbb{R} \times S^{3}$ a 1-forma $h(a, p) \lambda_{0}(p)$. As condições 3.1 e 3.2 definem uma família de 1-formas de contato em $S^{3}$ que deforma $\lambda_{E} \mathrm{em} \lambda$. A condição 3.3 diz que essa deformação é monótona e que para cada $a$ fixo a 1-forma $\lambda_{a}=h(a,.) \lambda_{0}$ é de contato, pois para $\lambda_{a}$ ser de contato basta $h(a, \cdot)>0$ o que é sempre verdade. A condição 3.4 diz que a restrição da 2 -forma $\left.d\left(h \lambda_{0}\right)\right)$ à $[-1,1] \times S^{3}$ é uma forma simplética. A variedade $\mathbb{R} \times S^{3}$ munida da 1 -forma $\tau=h \lambda_{0}$ é chamada 
de cobordismo simplético entre as variedades de contato $\left(S^{3}, \lambda\right)$ e $\left(S^{3}, \lambda_{E}\right)$. Associado à forma de contato $h(a,.) \lambda_{0}$ em $S^{3}$ temos um campo de Reeb que denotaremos por $X_{a}$.

Considere agora uma estrutura quase-complexa $J$ em $\mathbb{R} \times S^{3}$ determinada pelas seguintes condições:

$$
\begin{aligned}
& J(a, p) \frac{\partial}{\partial a}=X_{a}, \\
& J(a, p) X_{a}=-\frac{\partial}{\partial a},
\end{aligned}
$$

$$
J(a, p) \text { deixa o plano } \xi_{p}
$$

invariante e é compatível com $\left.d \tau\right|_{\xi}$

que é uma forma de área em $\xi_{p}$,

$$
\left.J(a, p)\right|_{\xi} \text { independe de a se }|a| \geq 2 .
$$

Observação 1: vendo $\xi$ como um fibrado vetorial simplético, $\left.J(a, p)\right|_{\xi}$ é compatível com a 2-forma $d\left(h(t, p) \lambda_{0}(p)\right)$ em cada fibra. Além disso 3.7 nos diz que $J(a, p)$ quando restrita à $[2,+\infty) \times S^{3}$ coincide com uma estrutura quase-complexa introduzida na simplectização de $\left(S^{3}, \lambda_{E}\right)$ por Hofer em [10]. Algo análogo vale para $J(a, p)$ restrito $(-\infty,-2] \times S^{3}$ para uma estrutura quase-complexa na simplectização de $\left(S^{3}, \lambda\right)$.

Por razões de transversalidade (para podermos aplicar o teorema da função implícita posteriormente) iremos considerar uma estrutura quase-complexa $\widehat{J}$ que relaxa as condições impostas sobre $J$ acima. $\widehat{J}$ satisfaz:

$$
\begin{aligned}
& \widehat{J}(a, p)=J(a, p) \text { se }|a| \geq 1 \\
& \widehat{J}(a, p) \text { é compatível com a forma simplética } d \tau \text { se }|a| \leq 1 .
\end{aligned}
$$

Definição 2. Seja $\Sigma$ uma superfície de Riemann compacta (com estrutura complexa j) e $\dot{\Sigma}$ a superfície obtida retirando-se de $\Sigma$ um conjunto finito de pontos. Considere $\widetilde{u}: \dot{\Sigma} \rightarrow \mathbb{R} \times S^{3}$. Dizemos que $\widetilde{u}$ é pseudo-holomorfa se vale: $\bar{\partial} \widetilde{u}=\frac{1}{2}(d \widetilde{u}+\widehat{J} \circ d \widetilde{u} \circ j)=0$ em todo ponto de $\dot{\Sigma}$. Nesse caso $\widetilde{u}$ é chamada curva pseudo-holomorfa. Escrevemos $\widetilde{u}(z)=(a(z), u(z))$ onde $a(z) \in \mathbb{R} e$ $u(z) \in S^{3}$.

Curvas pseudo-holomorfas em simplectizações de variedades de contato foram estudadas por Hofer em [10] para sua solução parcial da Conjectura de Weinstein. Para isso ele definiu uma energia (ver capítulo 3 de [18]) para curvas pseudo-holomorfas em simplectizações que é sempre não-negativa e mostrou que sempre que existe uma curva pseudo-holomorfa de energia finita e não-nula na simplectização de uma variedade de contato, o fluxo de Reeb nessa variedade possui uma órbita periódica. Como aqui estamos tratando de um cobordismo entre duas variedades de contato, a definição da energia é uma modificação natural da definição para simplectizações.

A energia $E(\widetilde{u})$ de $\widetilde{u}$ é dada por:

$$
\sup _{\phi \in \Lambda} \int_{\dot{\Sigma}} \widetilde{u}^{*} d(\phi \tau)
$$

onde $\Lambda=\left\{\phi: \mathbb{R} \rightarrow[0,1], \phi^{\prime} \geq 0, \phi(a)=\frac{1}{2}\right.$ se $\left.|a| \leq 1\right\}$. 
Observação 2: notamos que $\widehat{J}$ é compatível com $d(\phi \tau)$ no sentido de que $d(\phi \tau)(J v, J w)=$ $d(\phi \tau)(v, w)$.

$$
\begin{gathered}
d(\phi \tau)(v, \widehat{J} v)=\phi d \tau(v, \widehat{J} v)+d \phi \wedge \tau(v, \widehat{J} v)= \\
=\phi d \tau(v, \widehat{J} v)+\phi^{\prime}[d a(v) \tau(\widehat{J} v)-d a(\widehat{J} v) \tau(v)]= \\
=\phi d \tau(v, \widehat{J} v)+\phi^{\prime}\left[d a(v)^{2}+d a(\widehat{J} v)^{2}\right] \geq 0 .
\end{gathered}
$$

Vemos que nos pontos onde $d(\phi \tau)$ é simplética $\left(a \in[-1,1]\right.$ ou $\left.\phi^{\prime}>0\right)$ vale $d(\phi \tau)(v, \widehat{J} v)>0$. Para checar a segunda condição de compatibilidade dividimos em dois casos. Se $a \in[-\infty,-1] \cup$ $[1,+\infty]$ temos:

$$
\begin{gathered}
d(\phi \tau)(\widehat{J} u, \widehat{J} v)=\phi d \tau(\widehat{J} u, \widehat{J} v)+d \phi \wedge \tau(\widehat{J} u, \widehat{J} v)= \\
=\phi d \tau(u, v)+\phi^{\prime}[d a(\widehat{J} u) \tau(\widehat{J} v)-d a(\widehat{J} v) \tau(\widehat{J} u)]= \\
=\phi d \tau(u, v) \phi^{\prime}[d a(u) \tau(v)-d a(v) \tau(u)] .
\end{gathered}
$$

Se $a \in[-1,1]$ temos:

$$
d(\phi \tau)(\widehat{J} u, \widehat{J} v)=\phi d \tau(\widehat{J} u, \widehat{J} v)+d \phi \wedge \tau(\widehat{J} u, \widehat{J} v)=\phi d \tau(u, v)
$$

pois $d \phi=0$.

Podemos munir $\mathbb{R} \times S^{3}$ de uma métrica Riemanniana $g_{\widehat{J}}$ na qual $\widehat{J}$ é uma isometria no espaço tangente à cada ponto. Uma métrica assim pode ser definida tomando:

-em $[-1,1] \times S^{3} g_{\widehat{J}}(v, v)=d \tau(v, \widehat{J}(v))$

-em $\{\mathbb{R} \backslash[-1,1]\} \times S^{3}$ exigimos que $R_{a}$ e $\partial_{a}$ sejam vetores unitários, e que $\mathbb{R} R_{a}$ e $\mathbb{R} \partial_{a}$ e $\xi$ sejam subespaços ortogonais. Para vetores $w \in \xi$ definimos $g_{\widehat{J}}(w, w)=d \tau(w, \widehat{J} w)$.

\subsection{Comportamento de planos de energia finita}

A partir de agora estudaremos, nesse capítulo, planos e cilindros de energia finita em $\left(\mathbb{R} \times S^{3}, \widehat{J}\right)$, ou seja, o caso em que $\dot{\Sigma}$ é bi-holomorfo à $\mathbb{C}$ ou $\mathbb{R} \times S^{1}$ (com coordenadas cilíndricas $s+i t$ ) e suas estruturas complexas usuais. Veremos agora que todo plano de energia finita é assintótico à uma órbita periódica $P$ de $\left(S^{3}, \lambda_{E}\right)$ no seguinte sentido:

$u\left(R e^{i t}\right)$ converge a $P(t+c)$ (onde $c$ é uma constante) quando $R \rightarrow+\infty$.

$a\left(R e^{i t}\right) \rightarrow+\infty$ uniformemente em $t$ quando $R \rightarrow+\infty$.

O comportamento assintótico de planos de energia finita em simplectizações foi estudado em 14.

Para aplicar os resultados desse trabalho mostraremos que $a\left(R e^{i t}\right) \rightarrow+\infty$ uniformemente em $t$ quando $R \rightarrow+\infty$ o que nos garante que existe um $R_{0}>0$ tal que:

$$
|z|>R_{0} \Rightarrow \widetilde{u}(z) \in[2,+\infty) \times S^{3} .
$$

Como em $[2,+\infty), \widehat{J}$ coincide com a estrutura quase-complexa da simplectização de $\left(S^{3}, \lambda_{E}\right)$ podemos utilizar os resultados de [14] sobre o comportamento assintótico do plano $\widetilde{u}$.

Para isso começaremos estudando certas propriedades de planos de energia finita em $\left(\mathbb{R} \times S^{3}, \widehat{J}\right)$ e começamos com um lema. 
Lema 2. Seja $\widetilde{u}: \mathbb{C} \rightarrow\left(\mathbb{R} \times S^{3}, \widehat{J}\right)$ um plano pseudo-holomorfo com $0<E(\widetilde{u})<+\infty$. Então não existe $R \in \mathbb{R}$ tal que $\widetilde{u}(\mathbb{C}) \subset[-R, R] \times S^{3}$.

Demonstração: Suponha que exista $R>2 \in \mathbb{R}$ tal que $\widetilde{u}(\mathbb{C}) \subset[-R, R] \times S^{3}$. Escolha $\phi \in \Lambda$ tal que $\phi^{\prime}(a)>0$ se $a \in[-R,-1] \cup[1, R]$. Com essa propriedade $d(\phi \tau)$ é uma forma simplética em $[-R, R] \times S^{3}$. Como $\widetilde{u}$ é não-constante mostraremos que $\int_{\mathbb{C}} \widetilde{u}^{*} d(\phi \tau)>0$. Se $\widetilde{u}$ não é constante tome um ponto onde $\widetilde{u}_{s} \neq 0$. Então, pela observação 2 vale:

$$
\widetilde{u}^{*} d(\phi \tau)(\partial s, \partial t)=d(\phi \tau)\left(\widetilde{u}_{s}, \widetilde{u}_{t}\right)=d(\phi \tau)\left(\widetilde{u}_{s}, \widehat{J} \widetilde{u}_{s}\right)>0,
$$

já que $d(\phi \tau)$ é simplética.

Na variedade simplética compacta $\left([-R, R] \times S^{3}, d(\phi \tau)\right)$ temos então uma curva pseudo-holomorfa com integral $d(\phi \tau)$ finita. O teorema da remoção de singularidades de Gromov [8] nos diz então que podemos estender $\widetilde{u}$ de $\mathbb{C}$ à $S^{2} \cong \mathbb{C} \cup \infty$. Obtemos então uma esfera pseudo-holomorfa em uma variedade compacta simplética compacta. Mas para qualquer $\psi \in \Lambda, d(\psi \tau)$ é uma forma exata. Segue então do teorema de Stokes que para toda $\psi \in \Lambda, \int_{S^{2}} \widetilde{u}^{*} d(\psi \tau)=\int_{\partial S^{2}} \widetilde{u}^{*}(\psi \tau)=0$. Concluímos então que $E(\widetilde{u})=0$ e chegamos a uma contradição.

Provaremos agora que o gradiente de $\widetilde{u}$ é uniformemente limitado.

Iniciamos com dois lemas técnicos necessários para nossa análise de bubbling.

Lema 3. Seja $\widetilde{u}$ um plano pseudo-holomorfo não-constante em $\left(\mathbb{R} \times S^{3}, \widehat{J}\right)$. Então $\int_{\mathbb{C}} \widetilde{u}^{*} d \tau>0$.

Dividimos nossa prova em 2 casos:

(a) Existe um ponto de nosso plano pseudo holomorfo em $[-1,1] \times S^{3}$.

Nesse caso temos que existe $z \in \mathbb{C}$ tal que $d \widetilde{u}(z) \neq 0$ e $\widetilde{u}(z) \in[-1,1] \times S^{3}$ pois $\widetilde{u}$ é não constante. Introduzindo coordenadas complexas $s+i t$, temos para esse $z$ :

$$
\widetilde{u}^{*} d \tau(\partial s, \partial t)=d \tau\left(\widetilde{u}_{s}, \widetilde{u}_{t}\right)=d \tau\left(\widetilde{u}_{s}, \widehat{J} \widetilde{u}_{s}\right)>0,
$$

pois $\widetilde{u}_{s} \neq 0$ e $\widehat{J}$ é compatível com a forma simplética $d \tau$ em $[-1,1] \times S^{3}$. Como o integrando $\widetilde{u}^{*} d \tau$ é sempre não-negativo pela observação 2 , temos $\int \widetilde{u}^{*} d \tau>0$.

(b) Nosso plano pseudo-holomorfo está contido em $\{[-\infty,-1] \cup[1, \infty]\} \times S^{3}$.

Mas nesse caso como a estrutura quase-complexa coincide com $J$ temos, usando coordenadas complexas $s+i t$, que a equação $\bar{\partial} \widetilde{u}=0$ se escreve como:

$$
\begin{aligned}
& \pi_{a} u_{s}+J \pi_{a} u_{t}=0, \\
& \lambda_{a}\left(u_{s}\right)=-a_{t}, \\
& \lambda_{a}\left(u_{t}\right)=a_{s},
\end{aligned}
$$

onde $\pi_{a}: T S^{3} \rightarrow \xi$ é a projeção em $\xi$ ao longo do campo de Reeb $X_{a}$ dada por $\pi_{a}(x, v)=$ $\left(x, v-\lambda_{a}(v) X_{a}\right)$.

Observamos então que $\widetilde{u}^{*} \tau=\lambda_{a}\left(u_{s}\right) d s+\lambda_{a}\left(u_{t}\right) d t=-a_{t} d s+a_{s} d t$, donde:

$$
\widetilde{u}^{*} d \tau\left(\partial_{s}, \partial_{t}\right)=-\Delta a .
$$

Se a integral $\int \widetilde{u}^{*} d \tau=0$ temos necessariamente $\widetilde{u}^{*} d \tau=-\Delta a d s \wedge d t=0$, ou seja, a função $a$ é harmônica. Mas como $\widetilde{u} \subset\{[-\infty,-1] \cup[1, \infty]\} \times S^{3}$, temos que ou $a \leq-1$ ou $\geq+1$. Segue então do teorema de Liouville para funções harmônicas que $a$ é constante. Mas se $a$ é constante, isso implica que $\widetilde{u}(\mathbb{C}) \subset\{a(0)\} \times S^{3}$. Pelas fórmulas (3.15), (3.16) e (3.17) deve ser uma superfície sempre tangente à estrutura de contato, o que implica que $\widetilde{u}$ é constante. 
Corolário 1. Seja $\widetilde{v}: \mathbb{C} \backslash\{0\} \rightarrow\left(\mathbb{R} \times S^{3}, \widehat{J}\right)$ um cilindro pseudo-holomorfo não constante. Então $\int \widetilde{v}^{*} d \tau>0$.

Demonstração: Se $\widetilde{v}: \mathbb{C} \backslash\{0\} \rightarrow\left(\mathbb{R} \times S^{3}, \widehat{J}\right)$ satisfaz $\int \widetilde{v}^{*} d \tau=0$, definimos $\widetilde{u}: \mathbb{C} \rightarrow\left(\mathbb{R} \times S^{3}, \widehat{J}\right)$ $\operatorname{com} \widetilde{u}(z)=\widetilde{v}\left(e^{z}\right)$. Como a função exponencial é holomorfa de $\mathbb{C}$ em $\mathbb{C} \backslash\{0\}$ temos que $\widetilde{u}$ é um plano pseudo holomorfo e $\int \widetilde{u}^{*} d(\tau)=0$. Logo $\widetilde{u}$ é constante o que implica $\widetilde{v}$ constante.

Antes de prosseguir iremos enunciar um lema provado em [10] que será essencial nas análises de bubbling que faremos nesse capítulo. Para uma demonstração referimos a [18] páginas 71-72.

Lema de Hofer Seja $(X, d)$ um espaço métrico completo e $f: X \rightarrow \mathbb{R}$ uma função contínua. Para quaiquer $\epsilon_{0}>0$ e $x_{0} \in X$ existem $\epsilon_{0}^{\prime} \in\left(0, \epsilon_{0}\right]$ e $x_{0}^{\prime} \in \overline{B_{2 \epsilon_{0}}\left(x_{0}\right)}$ tais que:

$$
\begin{gathered}
f\left(x_{0}^{\prime}\right) \epsilon_{0}^{\prime} \geq f\left(x_{0}\right) \epsilon_{0}, \\
d\left(x, x_{0}^{\prime}\right) \leq \epsilon_{0}^{\prime} \Rightarrow f(x) \leq 2 f\left(x_{0}^{\prime}\right) .
\end{gathered}
$$

Prosseguimos agora com a seguinte:

Proposição 6. Seja $\widetilde{u}$ um plano de energia finita em $\left(\mathbb{R} \times S^{3}, \widehat{J}\right)$. Então existe $C>0$ tal que $\left|d \widetilde{u}\left(z_{k}\right)\right|<C$ para todo $z \in \mathbb{C}$, onde $\left|d \widetilde{u}\left(z_{k}\right)\right|$ é tomada em relação à métrica $g_{\widehat{J}}$

Observação: definimos $\left|d \widetilde{u}\left(z_{k}\right)\right|$ como $\max \left\{\left|d \widetilde{u}\left(z_{k}\right)(v)\right| ;|v|=1\right\}$. Calculamos $|v|$ segundo a métrica euclidiana em $\mathbb{C}$ que faz de $\mathbb{C}$ um espaço completo, e calculamos $\left|d \widetilde{u}\left(z_{k}\right)(v)\right|$ usando em $\mathbb{R} \times S^{3}$ a métrica $g_{\widehat{J}}$ que satisfaz: $g\left(X_{\lambda}, X_{\lambda}\right)=g\left(\partial_{a}, \partial_{a}\right)=1, w \in \xi g(w, w)=d \lambda(w, J w)$ para $w \in \xi$.

Demonstração: Por absurdo suponha que exista uma sequência de pontos $z_{k}$ tal que $\left|d \widetilde{u}\left(z_{k}\right)\right| \rightarrow$ $+\infty$. Tome então uma sequência $\delta_{k} \rightarrow 0$ tal que $\delta_{k}\left|d \widetilde{u}\left(z_{k}\right)\right| \rightarrow+\infty$. Então o Lema de Hofer aplicado à sequência $z_{k}$ em $\mathbb{C}$ (que é um espaço métrico completo) e à função contínua $\left|d \widetilde{u}\left(z_{k}\right)\right|$ nos diz que é possível escolher pontos $z_{k}^{\prime}$ e números $\epsilon_{k} \rightarrow 0$ com a seguinte propriedade:

$$
\begin{gathered}
R_{k}=\left|d \widetilde{u}\left(z_{k}^{\prime}\right)\right| \rightarrow+\infty, \\
R_{k} \epsilon_{k} \rightarrow+\infty, \\
|d \widetilde{u}(z)| \leq 2 R_{k} \text { se }\left|z-z_{k}^{\prime}\right|<\epsilon_{k} .
\end{gathered}
$$

Dividimos nossa demonstração agora em 2 casos:

(a) $\left|a\left(z_{k}^{\prime}\right)\right|$ é limitado.

Nesse caso definimos:

$$
\widetilde{v}_{k}(z)=\widetilde{u}\left(\frac{z}{R_{k}}+z_{k}^{\prime}\right)
$$

Observamos que $\left|d \widetilde{v}_{k}\right| \leq 2$ em $B_{R_{k} \epsilon_{k}}(0)$ e que a hipótese (a) nos garante que é possível (tomando uma subsequência se necessário) garantir que $\widetilde{v}_{k}(0)$ converge a um ponto de $\mathbb{R} \times S^{3}$. Isso, o fato de que $R_{k} \epsilon_{k} \rightarrow+\infty$ e regularidade elíptica nos permitem concluir que existe uma subsequência de $\widetilde{v}_{k}$ que converge a uma aplicação pseudo-holomorfa $\widetilde{v}: \mathbb{C} \rightarrow\left(\mathbb{R} \times S^{3}, \widehat{J}\right)$.

Como $R_{k} \epsilon_{k} \rightarrow+\infty$ temos: $\int_{\mathbb{C}} \widetilde{v}^{*} d \tau=\lim _{k \rightarrow+\infty} \int_{B_{R_{k} \epsilon_{k}}(0)} \widetilde{v}_{k}^{*} d \tau=\int_{B_{\epsilon_{k}}\left(z_{k}^{\prime}\right)} \widetilde{u}^{*} d \tau \rightarrow 0$ pois $\int_{\mathbb{C}} \widetilde{u}^{*} d \tau$ é finita. Segue então do Lema 2 que $\widetilde{v}$ é constante. Mas $|d \widetilde{v}(0)|=\lim _{k \rightarrow+\infty}\left|d \widetilde{v_{k}}(0)\right|=1$, o que contradiz o fato de que $\widetilde{v}$ é constante.

(b) $\left|a\left(z_{k}^{\prime}\right)\right|$ é ilimitado

Suporemos sem perda de generalidade que $\left|a\left(z_{k}^{\prime}\right)\right| \rightarrow+\infty$. 
Nesse caso definimos:

$$
\widetilde{v}_{k}(z)=\left(a\left(\frac{z}{R_{k}}+z_{k}^{\prime}\right)-a\left(z_{k}^{\prime}\right), u\left(\frac{z}{R_{k}}+z_{k}^{\prime}\right)\right) .
$$

Temos duas possibilidades:

1) $a\left(z_{k}^{\prime}\right) \rightarrow+\infty$.

2) $a\left(z_{k}^{\prime}\right) \rightarrow-\infty$.

Demonstraremos o caso 1), sendo que para 2) a demonstração é idêntica, trocando-se $\lambda_{E}$ por $\lambda$

1) A aplicação $\widetilde{v}_{k}$ é pseudo-holomorfa na simplectização de $\left(S^{3}, \lambda_{E}\right)$ se restringirmos o domínio a $B_{r_{k}}$ onde $r_{k}=\min \left\{R_{k} \epsilon_{k}, \frac{a\left(z_{k}^{\prime}\right)}{3}\right\}$. Sendo assim temos $r_{k} \rightarrow+\infty$ e a sequência $\widetilde{v}_{k}$ satisfaz:

$$
\begin{aligned}
\widetilde{v}_{k}: B_{r_{k}}(0) \rightarrow & \left(\mathbb{R} \times S^{3}, J_{\lambda_{E}}\right) \text { é pseudo-holomorfo } \\
& \left|d \widetilde{v}_{k}\right|_{B_{r_{k}}(0)} \mid \leq 2 \\
& \left|d \widetilde{v}_{k}(0)\right|=1 \\
& b_{k}(0)=0 .
\end{aligned}
$$

Então a regularidade elíptica nos permite concluir que existe uma subsequência de $\left.\widetilde{v}_{k}\right|_{B_{r_{k}}(0)}$ que converge a um plano pseudo-holomorfo de energia finita não constante $\widetilde{v}$. Entretanto, por um raciocínio idêntico ao exposto no caso a), vale $\int_{\mathbb{C}} \widetilde{v}^{*} d \lambda_{E}=0$.

Em [10] é provado que planos de energia finita na simplectização de uma variedade de contato $\left(M, \lambda^{\prime}\right)$ que não são constantes tem integral $d \lambda^{\prime}$ positiva. Aplicando esse resultado ao plano $\widetilde{v}$ na simplectização de $\left(S^{3}, \lambda_{E}\right)$ temos um absurdo. Isso finaliza a demonstração na hipótese 1). A demonstração na hipótese 2) é, como observamos anteriormente, análoga. Concluímos que o caso b) também leva a um absurdo, o que termina a prova de nossa proposição.

Comentário: Observamos que o resultado provado aqui vale para qualquer curva pseudoholomorfa em uma superfície de Riemann com furos $\dot{\Sigma}$ tomando-se em $\dot{\Sigma}$ uma métrica Riemanniana completa. Um caso especial que será necessário na continuação é que para $\widetilde{v}(R, \theta)=\widehat{u}\left(e^{2 \pi(R+i \theta)}\right)$, também existe $C>0$ tal que $|d \widetilde{v}|<C$. É imediato ver que se a sequência $R_{k}$ for limitada $\left|d \widetilde{v}\left(R_{k}, \theta_{k}\right)\right|$ é limitada. Logo, se $\left|d \widetilde{v}\left(R_{k}, \theta_{k}\right)\right|$ ficar arbitrariamente grande, podemos garantir (tomando uma subsequência se necessário) que $R_{k} \rightarrow+\infty$. Então, uma análise igual à feita na prova da Proposição acima leva à uma contradição, pela construção de um plano pseudo-holomorfo não-constante que tem integral $d \tau$ (ou $d \lambda$ ) nula.

Prosseguimos a análise dos planos de energia finita estudando o que ocorre agora quando $|z| \rightarrow$ $+\infty$. Nosso objetivo é mostrar que assim como no caso de simplectizações a parte real $a$ de $\widetilde{u}$ vai para $+\infty$ e que $u\left(R e^{i t}\right)$ converge uniformemente em $t$ a uma órbita periódica do elipsóide. Para isso começamos mostrando que $a(\mathbb{C})$ é inferiormente limitado.

Lema 4. Seja $\widetilde{u}$ um plano pseudo-holomorfo de energia finita. Então a é inferiormente limitada.

Demonstração: Suponha por absurdo que $a$ não é inferiormente limitada. Expressando $\widetilde{u}$ em coordenadas polares obtemos $\widetilde{v}: \mathbb{R} \times S^{1} \rightarrow \mathbb{R} \times S^{3}$ dado por:

$$
\widetilde{v}(R, \theta)=\widetilde{u}\left(e^{2 \pi(R+i \theta)}\right) .
$$

Como o mapa $(R, \theta) \mapsto e^{2 \pi(R+i \theta)}$ é holomorfo para a estrutura complexa $j$ usual no cilindro $\left(j\left(\partial_{\theta}\right)=-\partial_{R}\right)$ temos que o mapa $\widetilde{v}$ é pseudo-holomorfo. Pelo comentário acima temos que existe $C>0$ tal que $|d \widetilde{v}|<C$.

É possível então tomar uma sequência $\left(R_{k}, \theta_{k}\right) \rightarrow\left(+\infty, \theta^{*}\right)$ tal que $b\left(R_{k}, \theta_{k}\right) \rightarrow-\infty$, onde $\widetilde{v}=(b, v)$ e $\partial_{R} b\left(R_{k}, \theta_{k}\right)<0$. Como o gradiente de $\widetilde{v}$ é uniformemente limitado podemos ainda escolher a sequência $\left(R_{k}, \theta_{k}\right)$ de maneira que também valha $R_{k}-\frac{k}{C} \rightarrow+\infty$ e $b\left(R_{k}, \theta\right)<-k$. 
Definimos então a sequência de mapas:

$$
\widetilde{w}_{k}(R, \theta)=\left(b\left(R+R_{k}, \theta+\theta_{k}\right)-b\left(R_{k}, \theta_{k}\right), v\left(R+R_{k}, \theta+\theta_{k}\right)\right) .
$$

Observamos então que o mapa $\left.\widetilde{w}_{k}\right|_{\left[\frac{-k+2}{C}, \frac{k-2}{C}\right]}$ é pseudo-holomorfo na simplectização de $\left(S^{3}, \lambda\right)$ (com a estrutura quase-complexa $J_{\lambda}$ que definimos no fim inferior do cobordismo e que é invariante em relação à coordenada $\mathbb{R}$ ). Como a sequência $\widetilde{w}_{k}$ tem gradiente limitado e $\widetilde{w}_{k}(0,1) \in\{0\} \times S^{3}$ é possível tomar uma subsequência de $\widetilde{w}_{k}$ que converge a um cilindro pseudo-holomorfo $\widetilde{w}$ em $\left(\mathbb{R} \times S^{3}, J_{\lambda}\right)$ e que:

$$
\int_{\mathbb{R} \times S^{1}} \widetilde{w}^{*} d \lambda=\lim _{k \rightarrow+\infty} \int_{\left[\frac{-k+2}{C}, \frac{k-2}{C}\right] \times S^{1}} \widetilde{w}_{k}^{*} d \lambda=\lim _{k \rightarrow+\infty} \int_{\left[R_{k}-\frac{(k-2)}{C}, R_{k}+\frac{(k-2)}{C}\right] \times S^{1}} \widetilde{v}^{*} d \tau=0,
$$

onde usamos o fato de que o integrando $\widetilde{v}^{*} d \tau$ é não-negativo, $\widetilde{v}$ tem integral $d \tau$ finita e $R_{k}-\frac{k}{C} \rightarrow+\infty$ para garantir a igualdade à direita.

Os resultados de [10] nos dizem então que $\widetilde{w}=(g, w)$ é um cilindro sobre uma órbita periódica $x(t)$ de $X_{\lambda}$. Mas como $\partial_{R} g(0,1)=\lim _{k \rightarrow+\infty} \partial_{R} b\left(R_{k}, \theta_{k}\right) \leq 0$, $\widetilde{w}$ é da forma $\left(-T s+d, x\left(-T \theta+d^{\prime}\right)\right.$. Isso implica que existe uma sequência $r_{l} \rightarrow+\infty$ tal que:

$u\left(e^{2 \pi\left(r_{l}+i t\right)}\right)$ converge uniformemente em $t$ para $x\left(-T t+d^{\prime}\right)$. Então dado $\frac{-T}{10}$,tomamos $l$ suficientemente grande tal que:

$$
\int_{B_{e^{2 \pi r_{l}}(0)}} \widetilde{u}^{*} d \tau=\int_{|z|=e^{2 \pi r_{l}}(0)} \widetilde{u}^{*} \tau \leq \int_{S^{1}} x^{*} \tau-\frac{-T}{10}=\frac{-9 T}{10}<0 .
$$

Mas como $\widetilde{u}$ é pseudo-holomorfa deveríamos ter $\int_{B_{e^{2 \pi r_{l}}(0)}} \widetilde{u}^{*} d \tau>0$ para todo $l$, e portanto chegamos a um absurdo. Isso conclui a demonstração do lema.

Estamos agora prontos para provar o principal teorema desta seção.

Teorema 3. Seja $\widetilde{u}$ um plano pseudo-holomorfo de energia finita e não-constante em $\left(\mathbb{R} \times S^{3}, \widehat{J}\right)$. Então $\lim _{R \rightarrow+\infty} a\left(R e^{2 \pi i \theta}\right)=+\infty$.

Demonstração: Como a é inferiormente limitada, temos pelo Lema 1 que a não pode ser superiormente limitada, pois $\widetilde{u}$ não é constante. Usando novamente coordenadas polares, tomamos uma sequência $r_{k}$ com a seguinte propriedade $b_{k}\left(r_{k}, \theta\right)>k$ onde:

$$
(b(r, \theta), v(r, \theta))=\widetilde{v}(r, \theta)=\widetilde{u}\left(e^{2 \pi(r+i \theta)}\right) .
$$

Supondo, por absurdo, que a não satisfaz $\lim _{R \rightarrow+\infty} a\left(R e^{2 \pi i \theta}\right)=+\infty$, temos que $b(r,$.$) não$ tende uniformemente a $+\infty$ quando $r \rightarrow+\infty$, e portanto, existe $n_{0}>2$ que satisfaz:

para todo $r_{0}$, existe $r>r_{0}$ tal que $b(r, \theta)<n_{0} \forall \theta \in S^{1}$. Nessas condições é possível assumir que a sequência $r_{k}$ satisfaz também:

$$
\partial_{r} b\left(r_{k}, \theta_{k}\right)<0
$$

para algum $\theta_{k} \in S^{1}$ e $r_{k}-k \rightarrow+\infty$.

Definindo os mapas:

$$
\widetilde{w}_{k}(r, \theta)=\left(b\left(r+r_{k}, \theta+\theta_{k}\right)-b\left(r_{k}, \theta_{k}\right), v\left(r+r_{k}, \theta+\theta_{k}\right)\right) .
$$

Lembrando que $\exists C>0$ tal que $|d \widetilde{v}|<C$, temos que a restrição $\left.\widetilde{w}_{k}\right|_{\left[\frac{-k+n_{0}}{C}, \frac{k-n_{0}}{C}\right] \times S^{1}}$ define uma curva pseudo-holomorfa em $\left(\mathbb{R} \times S^{3}, J_{\lambda_{E}}\right)$. Assim, como $\widetilde{w}_{k}(0,1) \in\{0\} \times S^{3}$ existe uma subsequência 
de $\widetilde{w}_{k}$ que converge a um cilindro pseudo-holomorfo $\widetilde{w}$. Como $r_{k}-k \rightarrow+\infty$ e $\int_{\mathbb{R} \times S^{1}} \widetilde{v}^{*} d \tau$ é finita temos que:

$$
\int_{\mathbb{R} \times S^{1}} \widetilde{w}^{*} d \lambda_{E} \leq \lim _{k \rightarrow+\infty} \int_{\left[r_{k}-\frac{(k-2)}{C}, r_{k}+\frac{(k-2)}{C}\right] \times S^{1}} \widetilde{w}_{k}^{*} d \lambda_{E}=\lim _{k \rightarrow+\infty} \int_{\left[r_{k}-\frac{(k-2)}{C}, r_{k}+\frac{(k-2)}{C}\right] \times S^{1}} \widetilde{v}^{*} d \tau=0 .
$$

Então, por um raciocínio idêntico ao do final do Lema anterior prova-se que $\widetilde{w}(r, \theta)=(-T r+$ $\left.d, x\left(-T \theta+d^{\prime}\right)\right)$ onde $x(t)$ é uma órbita periódica do elipsóide, e acharíamos $r$ tal que:

$$
\int_{B_{r}(0)} \widetilde{u}^{*} d \tau<0
$$

o que é um absurdo. Isso finaliza a demonstração do teorema.

Usando agora que $a\left(r e^{2 \pi i \theta}\right) \rightarrow+\infty$ quando $r \rightarrow+\infty$ temos que a partir de um certo $r, \widetilde{u}$ restrito à $\mathbb{C} \backslash B_{r}(0)$ é um semi-cilindro pseudo-holomorfo contido em $[2,+\infty] \times S^{3}$. Como nessa região do cobordismo a estrutura quase-complexa é idêntica à da simplectização de $\left(\mathbb{R} \times S^{3}, \lambda_{E}\right)$ podemos considerar $\left.\widetilde{u}\right|_{\mathbb{C} \backslash B_{r}(0)}$ como um semi-cilindro pseudo-holomorfo na simpletização de $\left(\mathbb{R} \times S^{3}, \lambda_{E}\right)$.

É possível então aplicar a análise feita em [14] sobre o comportamento de semi-cilindros com energia finita e ilimitados. Como sabemos que $\lim _{R \rightarrow+\infty} a\left(R e^{2 \pi i \theta}\right)=+\infty$, e todas as órbitas periódicas de $X_{\lambda_{E}}$ são não-degeneradas essa análise nos dá que:

$$
u\left(R e^{2 \pi i t}\right) \rightarrow P(T t)
$$

quando $R \rightarrow+\infty$, onde $P(t)$ é uma órbita periódica de $X_{\lambda_{E}}$. Essa convergência é exponencial.

\subsection{A família de planos $\mu$}

Consideramos agora a seguinte família $\mu$ de curvas pseudo holomorfas:

$$
\begin{gathered}
\widetilde{u}: \mathbb{C} \rightarrow\left(\mathbb{R} \times S^{3}, \widehat{J}\right) \text { pseudo-holomorfo, } \\
\widetilde{u}=(a, u) \text { é assintótico à órbita periódica } P_{0}, \\
a(0)=\min \{a(\mathbb{C})\}, \\
\int_{\mathbb{D}} \widetilde{u}^{*} d \tau=T_{0}-\frac{\gamma_{0}}{2},
\end{gathered}
$$

onde $\mathbb{D}=\{z \in \mathbb{C} ;|z| \leq 1\}, P_{0}$ é a órbita periódica de ação mínima do campo $X_{\lambda_{E}}$ (que possui apenas 2 órbitas periódicas simples) e $T_{0}$ é seu período. Para definir $\gamma_{0}$, tome $\gamma_{1}$ como sendo o ínfimo dos períodos de órbitas periódicas de $X_{\lambda}$; e $\gamma_{2}$ como sendo o ínfimo da diferença $T_{0}-T$, onde $T$ é período de uma órbita periódica de $X_{\lambda}$ e $T<T_{0}$ ou, no caso, de isso nunca ocorrer gamma $_{2}=\frac{T_{0}}{2}$. A órbita $P_{0}$ tem índice de Conley-Zehnder 3 e a outra órbita tem índice de ConleyZehnder maior que 3. A não-degenerecência da forma $\lambda$ nos garante que $\gamma_{2}>0$. Definimos então $\gamma_{0}:=\min \left\{\gamma_{1}, \gamma_{2}\right\}$.

A condição $\int_{\mathbb{D}} \widetilde{u}^{*} d \tau=T_{0}-\frac{\gamma_{0}}{2}$ parece inicialmente arbitrária, mas sua importância vem do fato de que ela permite que tenhamos certo controle sobre o fenômeno de bubbling. Para ilustrar esse controle provamos um lema que é crucial em toda a análise que se segue.

Lema 5. Dado $\epsilon>0$, existe $C>0$ tal que $|d \widetilde{u}(z)|<C$, para todo $z \in \mathbb{C}$ com $|z| \geq 1+\epsilon$ e para todo $\widetilde{u}$ da família $\mu$.

Demonstração: Negando o lema supomos por absurdo que exista uma sequência de pontos $z_{k} \operatorname{com}\left|z_{k}\right| \geq 1+\epsilon$ e uma sequência de elementos $\widetilde{u}_{k} \in \mu$ satisfazendo: 


$$
\left|d \widetilde{u}_{k}\left(z_{k}\right)\right| \rightarrow+\infty .
$$

Então fazendo uma análise igual a da Proposição 6 geramos uma nova sequência de mapas $\widetilde{v}_{k}(z)=$ $\widetilde{u}_{k}\left(\frac{z}{R_{k}}+z_{k}\right)$ e uma sequência $R_{k} \rightarrow+\infty$ satisfazendo:

$$
\begin{gathered}
\left|d \widetilde{v}_{k}(0)\right|=1, \\
\left|d \widetilde{v}_{k}(z)\right| \leq 2 \text { se }|z| \leq R_{k} .
\end{gathered}
$$

Denotando $\widetilde{v}_{k}=\left(b_{k}, v_{k}\right)$, dividimos nossa demonstração agora em três casos:

(a) $b_{k}(0)$ é limitado.

Nesse caso argumentando como na Proposição 6 temos que existe uma subsequência de $\widetilde{v}_{k}$ que converge a uma aplicação pseudo-holomorfa $\widetilde{v}: \mathbb{C} \rightarrow\left(\mathbb{R} \times S^{3}, \widehat{J}\right)$. Mas:

$$
\int_{\mathbb{C}} \widetilde{v}^{*} d \tau \leq \lim _{k \rightarrow+\infty} \int_{B_{R_{k}}(0)} \widetilde{v}_{k}^{*} d \tau \leq \lim _{k \mapsto+\infty} \int_{\mathbb{C} \backslash B_{1+\frac{\epsilon}{2}}(0)} \widetilde{u}_{k}^{*} d \tau \leq \frac{\gamma_{0}}{2}
$$

Mas como vimos antes $\int_{\mathbb{C}} \widetilde{v}^{*} d \tau \leq \frac{\gamma_{0}}{2}$ é igual ao período da órbita periódica à qual $\widetilde{v}$ é assintótico. Mas $\widetilde{v}$ é assintótico a uma órbita periódica do elipsóide e isso implica $\int_{\mathbb{C}} \widetilde{v}^{*} d \tau \geq T_{0}$ e chegamos a uma contradição com $\int_{\mathbb{C}} \widetilde{v}^{*} d \tau \leq \frac{\gamma_{0}}{2}$.

(b) $b_{k}(0) \rightarrow-\infty$.

Nesse caso fazemos uma análise como acima mas consideramos as curvas pseudo-holomorfas $\widetilde{w}_{k}(z)=\left(b_{k}(z)-b_{k}(0), v_{k}(z)\right)$. Essa sequência é composta de curvas pseudo-holomorfas na simplectização de $\left(S^{3}, \lambda\right)$ para $|z| \leq \min \left\{R_{k}, \frac{\left|b_{k}(0)\right|}{4}\right\}$. Como $\min \left\{R_{k}, \frac{\left|b_{k}(0)\right|}{4}\right\} \rightarrow+\infty$ é possível tomar uma subsequência de $\widetilde{w}_{k}(z)$ que converge $\left(C^{\infty}\right.$ em partes compactas) para um plano pseudo-holomorfo $\widetilde{w}$ na simplectização de $\left(S^{3}, \lambda\right)$. Nesse caso esse plano tem área $d \lambda \int_{\mathbb{C}} \widetilde{w}^{*} d \lambda \leq \frac{\gamma_{0}}{2}$. Isso implicaria a existência de uma órbita periódica de $X_{\lambda}$ com período menor que $\gamma_{0}$ o que é um absurdo.

(c) $b_{k}(0) \rightarrow+\infty$.

A demonstração é análoga à acima mas o plano $\widetilde{w}$ é gerado na simplectização de $\left(S^{3}, \lambda_{E}\right)$. O absurdo ocorre pois a existência de $\widetilde{w}$ implicaria na existência de uma órbita periódica de $X_{\lambda_{E}}$ com período menor que $\gamma_{0}$.

O lema acima nos garante então que sempre que ocorre bubbling na família $\mu$ esse bubbling ocorre no disco unitário $\mathbb{D}$.

No artigo [11] é provada a existência de um plano pseudo holomorfo de energia finita mergulhado para a simplectização do elipsóide irracional $\left(S^{3}, \lambda_{E}\right)$ com propriedades especiais. Esse plano $\widetilde{u}_{0}$ é assintótico à menor órbita periódica do elipsóide $P_{0}$. De uma análise similar à feita na primeira seção desse capítulo temos que $a_{0}$ tem um mínimo, logo tomando uma translação vertical de $\widetilde{u}_{0}$ com $a(0)=3$ vemos que a família $\mu$ é não vazia. Como $\widetilde{u}_{0}$ é um mergulho e o operador $\bar{\partial}_{\widehat{J}}$ é um operador de Fredholm podemos usar o índice de Fredholm sobre uma determinada solução para calcular a dimensão local de $\mu$, seguindo o que é feito em [16]. Essa dimensão é dada pela seguinte fórmula, quando $\widetilde{u}$ é uma esfera pseudo-holomorfa furada:

$$
\operatorname{Fred}(\widetilde{u})=\mu_{C Z}^{+}-\mu_{C Z}^{-}-\chi\left(S^{2}\right)+\sharp \Gamma,
$$

onde $\mu_{C Z}^{+}$e $\mu_{C Z}^{-}$denotam a soma dos índices de Conley-Zehnder dos furos positivos e negativos respectivamente, e $\sharp \Gamma$ denota o número de furos de $\widetilde{u}$. Segue que no nosso caso de um plano 
pseudo-holomorfo mergulhado assintótico à $P_{0}$ que satisfaz $\mu_{C Z}\left(P_{0}\right)=3$, temos:

$$
\operatorname{Fred}(\widetilde{u})=\mu_{C Z}\left(P_{0}\right)-1=2 .
$$

Observação: notamos que todos os planos de $\mu$ que estão na mesma componente conexa por caminhos de $\widetilde{u}_{0}$ são mergulhos. Esse fato que será demonstrado no Apêndice A se baseia na construção de um índice de auto-intersecção para curvas pseudo-holomorfas que é invariante por homotopia, e que quando se anula implica que a curva é um mergulho. Diversas outras propriedades importantes de curvas pseudo-holomorfas, como o fato da projeção em $S^{3}$ ser transversal ao fluxo podem ser codificadas a partir de índices invariantes por homotopia e isso garante que essas propriedades são mantidas para deformações de uma curva. Para não interromper nossa construção iremos tratar desses índices no Apêndice A.

Prosseguimos no estudo da família $\mu$.

\subsubsection{Compacidade da família $\mu_{[\alpha, \beta]}$}

Seja $\mu_{[\alpha, \beta]}$ a seguinte família de planos pseudo holomorfos:

$$
\mu_{[\alpha, \beta]}=\{\widetilde{u} \in \mu \text { e } a(0) \in[\alpha, \beta]\} .
$$

Lema 6. Existe uma constante $C_{[\alpha, \beta]}>0$ tal que $|d \widetilde{u}|<C$ para todo $\widetilde{u} \in \mu_{[\alpha, \beta]}$.

Suponha por absurdo que não existe $C_{[\alpha, \beta]}$ tal que $\left|d \widetilde{u}_{k}\right|<C_{[\alpha, \beta]}$ para todo $\widetilde{u} \in \mu_{[\alpha, \beta]}$. Então existem sequências $z_{k}$ e $\widetilde{u}_{k}$ tais que:

$$
\left|d \widetilde{u}_{k}\left(z_{k}\right)\right| \rightarrow+\infty
$$

Notamos que, pelo Lema 4 , temos que $\lim \sup _{k \rightarrow \infty}\left|z_{k}\right| \leq 1$. É possível então tomar uma subsequência convergente $z_{k} \rightarrow z_{0}$, ou seja, $z_{0}$ é ponto de bubbling.

Usando o Lema de Hofer é possível então achar uma sequência $z_{k}^{\prime} \rightarrow z_{0}$ tal que :

$$
\begin{gathered}
\left|d \widetilde{u}_{k}\left(z_{k}^{\prime}\right)\right| \rightarrow+\infty, \\
\left|d \widetilde{u}_{k}(z)\right| \leq 2\left|d \widetilde{u}_{k}\left(z_{k}^{\prime}\right)\right| \forall z \in B_{\epsilon_{k}}\left(z_{k}^{\prime}\right), \\
\epsilon_{k}\left|d \widetilde{u}_{k}\left(z_{k}^{\prime}\right)\right| \rightarrow+\infty .
\end{gathered}
$$

Dividimos a demonstração em dois casos:

a) $a_{k}\left(z_{k}^{\prime}\right) \rightarrow+\infty$

Nesse caso, uma análise similar à feita no caso b) da Proposição 6 nos permite construir um plano pseudo-holomorfo $\widetilde{u}$ de energia finita na simplectização de $\left(S^{3}, \lambda_{E}\right)$. A principal diferença é que no atual caso $\int_{\mathbb{C}} \widetilde{u}^{*} d \lambda_{E}$ pode ser não nula.

Esse plano é assintótico a uma órbita periódica de $X_{\lambda_{E}}$. Temos que:

$$
\int_{\mathbb{C}} \widetilde{u}^{*} d \lambda_{E} \geq T_{0}
$$

Isso significa que $\widetilde{u}$ tem ao menos $T_{0}$ de integral $d \tau$. Como os elementos da família $\mu$ tem integral $d \tau$ igual à $T_{0}$ temos que $\widetilde{u}$ toma toda a integral $d \tau$ no bubbling.

b) $a_{k}\left(z_{k}^{\prime}\right)$ é limitado

Nesse caso uma análise similar à feita no caso a) da Proposição 6 nos permite construir um plano pseudo-holomorfo $\widetilde{u} \mathrm{em}\left(\mathbb{R} \times S^{3}, \widehat{J}\right)$. Novamente $\widetilde{u}$ tem de ser assintótico à órbita periódica 
$P_{0}$ de menor ação, pois como observamos só há $\int_{\mathbb{C}} \widetilde{u}^{*} d \tau \leq T_{0}$ pois os elementos da família $\mu$ tem integral $d \tau$ igual à $T_{0}$. Reunificamos agora a demonstração.

a) e b) mostram que para todo ponto de bubbling geramos um plano pseudo-holomorfo que rouba $T_{0}$ da integral $d \tau$. Como só existe $T_{0}$ disponível, existe apenas 1 ponto de bubbling. Chamamos $z_{0}$ esse ponto de bubbling.

Afirmamos: $\left|z_{0}\right|=1$

Do Lema 4 sabemos que $\left|z_{0}\right| \leq 1$. Se $\left|z_{0}\right|<1$ então o plano pseudo-holomorfo $\widetilde{u}$ produzido no bubbling pode roubar no máximo a integral $d \tau$ contida no disco unitário $\mathbb{D}$, pois a partir de um certo $k, B_{\epsilon_{k}}(0) \subset \mathbb{D}$. Logo:

$$
\int_{\mathbb{C}} \widetilde{u}^{*} d \tau \leq \limsup _{k \rightarrow+\infty} \int_{B_{\epsilon_{k}}(0)} \widetilde{u}_{k}^{*} d \tau \leq \limsup \int_{\mathbb{D}} \widetilde{u}_{k}^{*} d \tau=T_{0}-\frac{\gamma_{0}}{2} .
$$

Como $\widetilde{u}$ é assintótico a uma órbita periódica de $X_{\lambda_{E}}$ a equação acima implicaria que existe uma órbita periódica $X_{\lambda_{E}}$ com ação menor ou igual a $T_{0}-\frac{\gamma_{0}}{2}$, o que é um absurdo. Isso finaliza a demonstração da afirmação.

Em cada compacto de $\mathbb{C} \backslash\left\{z_{0}\right\}$ o gradiente da sequência $\widetilde{u}_{k}$ é uniformemente limitado. Como $z_{0} \neq 0$ temos que $\widetilde{u}_{k}\left(\mathbb{C} \backslash\left\{z_{0}\right\}\right)$ tem um ponto em $[\alpha, \beta] \times S^{3}$. Usando então o teorema de Arzelá-Ascoli existe uma subsequência de $\widetilde{u}_{k}$ que converge localmente em $\mathbb{C} \backslash\left\{z_{0}\right\}$ a um cilindro pseudo-holomorfo $\widetilde{v}$ em $\left(\mathbb{R} \times S^{3}, \widetilde{J}\right)$, e como o bubbling em $z_{0}$ roubou toda a integral $d \tau$ disponível:

$$
\int_{\mathbb{C} \backslash\left\{z_{0}\right\}} \widetilde{v}^{*} d \tau=0
$$

Pelo Corolário 1, isso implica que $\widetilde{v}$ é constante, mas como:

$$
\int_{|z|=1+\epsilon} \widetilde{v}^{*} \tau=\lim \int_{|z|=1+\epsilon} \widetilde{u}_{k}^{*} \tau \geq T_{0}-\frac{\gamma_{0}}{2},
$$

$\widetilde{v}$ é não-constante, o que é absurdo. Isso finaliza a prova do lema.

O Lema 5 implica que a família $\mu_{[\alpha, \beta]}$ é compacta. Para vermos isso tomemos uma sequência $\widetilde{u}_{k} \in \mu_{[\alpha, \beta]}$. Como $|d \widetilde{u}|<C$ temos que em cada compacto de $\mathbb{C}$ a sequência $\widetilde{u}_{k}$ é equicontínua. Em compactos a sequência $\widetilde{u}_{k}$ è uniformemente limitada. A hipótese de que $a_{k}(0) \in[\alpha, \beta]$ nos garante que a sequência $\widetilde{u}_{k}$ é totalmente limitada, e por isso satisfaz as hipóteses do teorema de Arzelá-Ascoli. Segue que $\widetilde{u}_{k}$ tem uma subsequência convergente e pela regularidade elíptica tem de ser um plano pseudo-holomorfo $\widetilde{u} \mathrm{em}\left(\mathbb{R} \times S^{3}, \widehat{J}\right)$. Como $\widetilde{u}$ é obtido como um limite de elementos da família $\mu$ temos $\int_{\mathbb{C}} \widetilde{u}^{*} d \tau \leq T_{0}$. Como $\widetilde{u}$ é assintótico a uma órbita periódica do elipsóide, ele tem de ser assintótico a $P_{0}$ que é a órbita de ação mínima, e portanto $\widetilde{u} \in \mu$.

\subsection{Não limitação da família $\mu$}

Usando a compacidade demonstrada na seção anterior provaremos que existe uma sequência $\widetilde{u}_{k}$ de elementos de $\mu$ com $a_{k}(0) \rightarrow-\infty$. Em outras palavras:

Teorema 4. $\inf \{a(0), \widetilde{u} \in \mu\}=-\infty$

Demonstração: Suponha por absurdo que isso não ocorra e seja inf $\{a(0), \widetilde{u} \in \mu\}=\alpha>-\infty$. Então existe uma sequência $\widetilde{u}_{k} \in \mu$ com:

$$
\lim _{k \rightarrow+\infty} a_{k}(0)=\alpha
$$


Tomemos então uma subsequência $\widetilde{u}_{k}^{\prime}$ de $\widetilde{u}_{k}$ satisfazendo:

$$
\widetilde{u}_{k}^{\prime} \in \mu_{[\alpha, \beta]}
$$

para algum $+\infty>\beta>\alpha$.

Pela compacidade de $\mu_{[\alpha, \beta]}$ a sequência $\widetilde{u}_{k}^{\prime}$ tem uma subsequência convergente a um plano pseudo-holomorfo $\widetilde{u} \in \mu$. Esse plano claramente satisfaz $a(0)=\alpha$.

Até agora provamos que o ínfimo $\alpha$ é na realidade um mínimo pois ele tem que ser realizado como $a(0)$ de algum plano $\widetilde{u} \in \mu$. No Apêndice A é demonstrado que todos elementos de $\mu$ são mergulhos. Isso nos permite aplicar o seguinte Teorema 1.6 de [16]:

Teorema 5. Seja $\widehat{J}$ uma estrutura quase-complexa $\left(\mathbb{R} \times M^{3}\right)$ que é genérica entre as estruturas quase-complexas compativeis com $d \tau$. Seja $\widetilde{u}: \mathbb{C} \rightarrow\left(\mathbb{R} \times M^{3}, \widehat{J}\right)$ um plano pseudo-holomorfo mergulhado assintótico a uma órbita periódica não degenerada e simplesmente coberta $P$ com $\mu_{C Z}(P)=3$. Então existe uma aplicação $\Phi: \mathbb{D} \times \mathbb{C} \rightarrow \mathbb{R} \times M^{3}$ tal que:

$$
\Phi(y, \cdot): \mathbb{C} \rightarrow\left(\mathbb{R} \times M^{3}, \widehat{J}\right),
$$

é um plano pseudo holomorfo mergulhado assintótico a $P$ e $\Phi(0, \cdot)=\widetilde{u}$. Além disso $\Phi$ é um difeomorfismo de $\mathbb{D} \times \mathbb{C}$ sobre uma vizinhança tubular do plano $\widetilde{u}(\mathbb{C})$ em $\mathbb{R} \times M^{3}$.

Decorre desse teorema que existe uma vizinhança de $\widetilde{u}(\mathbb{C})$ em $\mathbb{R} \times S^{3}$ que é folheada por planos pseudo-holomorfos de $\mu$. Essa vizinhança de $\widetilde{u}(\mathbb{C})$ certamente contém pontos do conjunto $\{\alpha-\epsilon\} \times S^{3}$ para algum $\epsilon>0$. Como essa vizinhança é folheada por planos elementos de $\mu$ existe $\widetilde{u}_{0} \in \mu$ tal que de $\widetilde{u}_{0}(\mathbb{C}) \cap\left(\{\alpha-\epsilon\} \times S^{3}\right) \neq \emptyset$. Segue que $\widetilde{u}_{0}$ necessariamente satisfaz:

$$
a(0) \leq \alpha-\epsilon .
$$

Mas isso contradiz que $\inf \{a(0), \widetilde{u} \in \mu\}=\alpha$. Esse absurdo completa a demonstração do teorema.

Concluímos então que a família $\mu$ não é limitada inferiormente. Estudaremos agora o que ocorre quando tomamos uma sequência $\widetilde{u}_{k}$ em $\mu$ com $a_{k}(0)$ tendendo a $-\infty$. Iniciamos com uma importante proposição que nos permite um controle do comportamento dessa sequência.

Proposição 7. Seja $\widetilde{u}_{k}$ uma sequência em $\mu$ satisfazendo $a_{k}(0) \rightarrow-\infty$. Então para todo z com $|z|>1$, temos que o conjunto $\left\{a_{k}(z) ; k \in \mathbb{N}\right\}$ é limitado.

Demonstração: Suponha por absurdo que não vale $\left\{a_{k}(z) ; k \in \mathbb{N}\right\}$ limitado para algum $z \in \mathbb{C}$ com $|z|>1$. Temos 2 possibilidades:

a) existe uma subsequência $\widetilde{u}_{l}$ da sequência original com $a_{l}(z) \rightarrow+\infty$.

b) existe uma subsequência $\widetilde{u}_{l}$ da sequência original com $a_{l}(z) \rightarrow-\infty$.

Caso a)

$\mathrm{O}$ fato de $a_{l}(0) \rightarrow-\infty$ junto com a) implica que ocorre bubbling na sequência $\widetilde{u}_{l}$. Para provar isso ligamos 0 e $z$ por um caminho $\gamma$ de $[0,1]$ em $\mathbb{C}$. Temos então que $\left|a_{l}(0)-a_{l}(z)\right| \rightarrow+\infty$ e a desigualdade do valor médio implica então que $\left(a_{l} \circ \gamma\right)^{\prime}$ tem de ficar arbitrariamente grande. Como $\gamma$ tem domínio compacto isso implica que a norma do gradiente de $a_{l}$ tem de ficar arbitrariamente grande, e portanto tem de ocorrer bubbling. Notamos ainda que a origem 0 deve ser um ponto de bubbling. Do contrário seria possível ligar 0 e $z$ (que nunca é ponto de bubbling) por um caminho evitando todos os pontos de bubbling (que são finitos). Nesse caminho o gradiente $\nabla a_{l}$ deveria então ser limitado, o que contraria o raciocínio que acabamos de desenvolver.

Cada ponto de bubbling dá origem a um plano de energia finita em $\left(\mathbb{R} \times S^{3}, J_{\lambda_{E}}\right),\left(\mathbb{R} \times S^{3}, J_{\lambda}\right)$ ou $\left(\mathbb{R} \times S^{3}, \widehat{J}\right)$, e esses planos tem portanto integral $d \tau$ maior que $\gamma_{0}$. A soma das integrais nesses 
planos não pode exceder $T_{0}$, portanto o número de pontos de bubbling é finito. Chamemos $\Gamma$ o conjunto dos pontos de bubbling de $\widetilde{u}_{l}$. Como vimos anteriormente no conjunto $\mathbb{C} \backslash \Gamma$ o gradiente da sequência $\widetilde{u}_{l}$ é uniformemente limitado. Logo em cada compacto $K$ de $\mathbb{C} \backslash \Gamma$ é possível tomar $l_{0}$ tal que $\widetilde{u}_{l}(K) \subset[2,+\infty] \times S^{3}$.

É possível então tomar uma subsequência de $\widetilde{u}_{l}$ denotada por $\widetilde{v}_{l}: K_{l} \rightarrow\left(\mathbb{R} \times S^{3}, J_{\lambda_{E}}\right)$ de curvas pseudo-holomorfas onde $K_{l}$ são compactos de $\mathbb{C} \backslash \Gamma, K_{l} \subset K_{l+1}$ e $\bigcup_{l \geq 0} K_{l}=\mathbb{C} \backslash \Gamma$, e $\widetilde{v}_{l}$ é dado pela fórmula:

$$
\left(b_{l}(w), v_{l}(w)\right)=\widetilde{v}_{l}(w)=\left(a_{l}(w)-a_{l}(z), u(z)\right) .
$$

Como temos que o gradiente de $\widetilde{v}_{l}$ é uniformemente limitado em compactos de $\mathbb{C} \backslash \Gamma$ e $b_{l}(z)=0$, a regularidade elíptica nos garante a existência de uma subsequência de $\widetilde{v}_{l}$ que converge a uma curva pseudo-holomorfa $\widetilde{v}: \mathbb{C} \backslash \Gamma \rightarrow\left(\mathbb{R} \times S^{3}, J_{\lambda_{E}}\right)$.

Pensando o domínio da curva pseudo-holomorfa $\widetilde{v}$ com uma esfera furada $(\mathbb{C} \bigcup\{\infty\}) \backslash(\Gamma \bigcup\{\infty\})$, ela tem como furos o conjunto $\Gamma \bigcup\{\infty\}$. Perto de cada um desses furos ela é assintótica à uma órbita periódica do elipsóide. Será provado na próxima subseção que todos os furos são negativos exceto $\infty$, ou seja, que $b(w) \rightarrow+\infty$ quando $|w| \rightarrow+\infty$, e $b(w) \rightarrow-\infty$ quando $w \rightarrow z_{j} \in \Gamma$. Mas a teoria de Fredholm nos permite calcular o índice de Fredholm de $\widetilde{v}$ segundo a fórmula:

$$
\operatorname{Fred}(\widetilde{v})=\mu_{C Z}\left(P_{0}\right)-\mu^{-}(\widetilde{v})-\chi\left(S^{2}\right)+1+\# \Gamma=2+\# \Gamma-\mu^{-}(\widetilde{v}) \leq 2+\# \Gamma-3 \# \Gamma=2-2 \# \Gamma,
$$

onde $\mu^{-}(\widetilde{v})$ é a soma dos índices de Conley-Zehnder dos furos negativos. Na desigualdade acima utilizamos que o índice de Conley-Zehnder de todas órbitas do elipsóide é maior ou igual à 3 , e na primeira igualdade usamos que todos os furos em $\Gamma$ são negativos.

O índice de Fredholm de uma curva pseudo-holomorfa é sempre não-negativo para uma escolha genérica de $J_{\lambda_{E}}$ e isso implica que $\# \Gamma \leq 1$. Como 0 é ponto de bubbling concluímos que $\# \Gamma=$ 1. Para que o índice de Fredholm seja não-negativo é também preciso que o furo negativo seja assintótico à uma órbita que tenha índice de Conley-Zehnder 3 o que força essa órbita a ser $P_{0}$. Assim $\widetilde{v}$ é um cilindro pseudo-holomorfo e fazemos a seguinte estimativa:

$$
\begin{array}{r}
\int_{\mathbb{C} \backslash\{0\}} \widetilde{v}^{*} d \lambda_{E}=\lim _{k \rightarrow+\infty} \int_{B_{k}(0) \backslash B_{\frac{1}{k}}(0)} \widetilde{v}^{*} d \lambda_{E}= \\
\lim _{k \rightarrow+\infty}\left(\int_{|w|=k} \widetilde{v}^{*} \lambda_{E}-\int_{|w|=\frac{1}{k}} \widetilde{v}^{*} \lambda_{E}\right)=T_{0}-T_{0}=0 .
\end{array}
$$

Isso implica que $\widetilde{v}$ tem de ser um cilindro sobre a órbita periódica $P_{0}$. Em particular vale que $\int_{S^{1}} \widetilde{v}^{*} \lambda_{E}=T_{0}$. Mas temos por outro lado a seguinte estimativa que vem da definição da família $\mu$ :

$$
\int_{|w|=1} \widetilde{v}^{*} \lambda_{E}=\lim _{l \rightarrow+\infty} \int_{S^{1}} \widetilde{v}_{l}^{*} \lambda_{E}=\int_{B_{1}(0)} \widetilde{u}^{*} d \tau=T_{0}-\frac{\gamma_{0}}{2} .
$$

Essas duas estimativas se contradizem e concluímos que a) gera um absurdo.

Caso b)

Suponha então que $a_{l}(z) \rightarrow-\infty$. Sabemos que fixando $l, a_{l}(w) \rightarrow+\infty$ quando $|w| \rightarrow+\infty$, $\operatorname{logo}$ existe $R_{l}>|z|$ tal que $a\left(R_{l}\right)=0$. Observamos que $R_{l} \rightarrow+\infty$ quando $l \rightarrow+\infty$ pois:

$$
\lim _{l \rightarrow+\infty}\left|a_{l}(z)-a_{l}\left(R_{l}\right)\right|=+\infty
$$

e como o gradiente da sequência $\widetilde{v}_{l}$ é uniformemente limitado em $\mathbb{C} \backslash \overline{\mathbb{D}}$ (onde se encontram $z$ e $R_{l}$ ) isso implica que a sequência $R_{l}$ escapa de qualquer compacto, caso contrário $\left|a_{l}(z)-a_{l}\left(R_{l}\right)\right|$ seria uma sequência limitada. 
Definimos então uma nova sequência de mapas $\widetilde{v}_{l}: \mathbb{C} \rightarrow\left(\mathbb{R} \times S^{3}\right)$ por:

$$
\widetilde{v}_{l}(w)=\left(b_{l}(w), v_{l}(w)\right)=\left(a_{l}\left(w R_{l}\right), u\left(z R_{l}\right)\right) .
$$

Vale que $\lim _{l \rightarrow+\infty}\left|b_{l}(1)-b_{l}(0)\right|=\lim _{l \rightarrow+\infty}\left|a_{l}\left(R_{l}\right)-a_{l}(0)\right|=+\infty$. Isso implica, usando a desigualdade do valor médio, que o gradiente de $\widetilde{v}_{l}$ assume valores arbitrariamente grandes quando $l \rightarrow+\infty$. Por um raciocínio idêntico ao usado no Caso a) para mostrar que 0 é ponto de bubbling, no atual caso novamente 0 é ponto de bubbling da sequência $\widetilde{v}_{l}$. Temos 3 possibilidades para o plano de energia finita $\widetilde{u}$ produzido nesse bubbling:

1) $\widetilde{u}$ é plano de energia finita em $\left(\mathbb{R} \times S^{3}, J_{\lambda_{E}}\right)$.

Nesse caso, $\widetilde{u}$ tem energia $T_{0}$ e por isso, rouba toda a integral $d \tau$ disponível na sequência $\widetilde{v}_{l}$, o que implica que 0 é o único ponto de bubbling. Segue que a sequência $\widetilde{v}_{l}$ tem gradiente limitado em compacto de $\mathbb{C} \backslash\{0\}$, e isso junto com o fato de $b_{l}(1)=0$ para todo $l$ garante que existe uma sequência $\widetilde{v}_{l}$ que converge a um cilindro pseudo holomorfo $\widetilde{v}$ em $\left(\mathbb{R} \times S^{3}, \widehat{J}\right)$. Como toda a integral $d \tau$ foi concentrada em $\widetilde{u}$ ocorre:

$$
\int_{\mathbb{C} \backslash\{0\}} \widetilde{v}^{*} d \tau=0 .
$$

Mas como não existem cilindros pseudo-holomorfos não-constantes de integral $d \tau$ nula em $(\mathbb{R} \times$ $\left.S^{3}, \widehat{J}\right)$, temos que $\widetilde{v}$ é constante.

Por outro lado temos a estimativa.

$$
\begin{array}{r}
\int_{S^{1}} \widetilde{v}^{*} \tau=\lim _{l \rightarrow+\infty} \int_{S^{1}} \widetilde{v}_{l}^{*} \tau=\lim _{l \rightarrow+\infty} \int_{B_{1}(0)} \widetilde{v}_{l}^{*} d \tau= \\
=\lim _{l \rightarrow+\infty} \int_{B_{R_{l}}(0)} \widetilde{u}_{l}^{*} d \tau \geq T_{0}-\frac{\gamma_{0}}{2}
\end{array}
$$

Como $\widetilde{v}$ deveria ser constante a última fórmula nos conduz a uma contradição.

2) $\widetilde{u}$ é um plano pseudo-holomorfo em $\left(\mathbb{R} \times S^{3}, \widehat{J}\right)$.

A demonstração desse caso é totalmente análoga ao do caso anterior porque novamente o plano $\widetilde{u}$ tem energia $T_{0}$ e por isso, rouba toda a energia $d \tau$ disponível na sequência $\widetilde{v}_{l}$.

3) $\widetilde{u}$ é plano pseudo-holomorfo em $\left(\mathbb{R} \times S^{3}, J_{\lambda}\right)$.

Nesse caso $\widetilde{u}$ rouba ao menos $\gamma_{0}$ de energia. Além disso poderiam ocorrer outros pontos de bubbling. Mas na sequência $\widetilde{v}_{l}$, dado $\epsilon>0$ não pode ocorrer bubbling fora de $B_{\epsilon}(0)$, pois:

$$
\lim _{l \rightarrow+\infty} \int_{\mathbb{C} \backslash B_{\epsilon}(0)} \widetilde{v}_{l}^{*} d \tau=\lim _{l \rightarrow+\infty} \int_{\mathbb{C} \backslash B_{R_{l} \epsilon}(0)} \widetilde{u}_{l}^{*} d \tau \leq \frac{\gamma_{0}}{2},
$$

implica que não há energia suficiente fora de $B_{\epsilon}(0)$ para produzir um plano de energia finita.

Como 0 é o único ponto de bubbling da sequência $\widetilde{v}_{l}$, o gradiente de $\widetilde{v}_{l}$ é limitado em compactos de $\mathbb{C} \backslash\{0\}$ e isso junto com $b_{l}(1)=1$ implica a existência de uma subsequência de $\widetilde{v}_{l}$ que converge a um cilindro pseudo-holomorfo $\widetilde{v}$ em $\left(\mathbb{R} \times S^{3}, \widehat{J}\right)$. Tendo que $\widetilde{u}$ rouba $T$ de energia $\left(T<T_{0}\right.$ caso contrário teríamos um absurdo como visto no caso 1) e 2)), e lembrando da definição de $\gamma_{0}$ vale:

$$
\int_{\mathbb{C} \backslash\{0\}} \widetilde{v}^{*} d \tau=T_{0}-T \geq \gamma_{0}
$$

pois:

$\inf \left\{T-T_{0} ; T<T_{0}\right.$ e $T$ período de alguma órbita periódica de $\left.X_{\lambda}\right\}=\gamma_{1} \geq \gamma_{0}$. 
Por outro lado vale o seguinte limite:

$$
\int_{\mathbb{C} \backslash\{0\}} \widetilde{v}^{*} d \tau=\lim _{n \rightarrow+\infty} \int_{B_{n}(0) \backslash B_{\frac{1}{n}}(0)} \widetilde{v}^{*} d \tau,
$$

e para todo $n$ temos:

$$
\begin{gathered}
\int_{B_{n}(0) \backslash B_{\frac{1}{n}}(0)} \widetilde{v}^{*} d \tau=\lim _{l \rightarrow+\infty} \int_{B_{n}(0) \backslash B_{\frac{1}{n}}(0)} \widetilde{v}_{l}^{*} d \tau= \\
=\lim _{l \rightarrow+\infty} \int_{B_{R_{l} n}(0) \backslash B_{R_{l} \frac{1}{n}}(0)} \widetilde{u}_{l}^{*} d \tau \leq \int_{\mathbb{C} \backslash B_{1}(0)} \widetilde{u}_{l}^{*} d \tau \leq \frac{\gamma_{0}}{2}
\end{gathered}
$$

Isso implica $\int_{\mathbb{C} \backslash\{0\}} \widetilde{v}^{*} d \tau \leq \frac{\gamma_{0}}{2}$. Mas então, as duas estimativas para $\int_{\mathbb{C} \backslash\{0\}} \widetilde{v}^{*} d \tau$ se contradizem e temos um absurdo.

Isso conclui a demonstração de 3) e também do caso b)

Como consequência da proposição anterior temos um maior controle no fenômeno de bubbling da sequência $\widetilde{u}_{k}$. Seja $\Gamma$ o conjunto dos pontos de bubbling da sequência $\widetilde{u}_{k}$. Em compactos contidos no complementar de $\Gamma$ o gradiente é uniformemente limitado, e a proposição anterior garante que pontos com norma maior que 1 ficam presos em um conjunto compacto de $\mathbb{R} \times S^{3}$. Com essas informações é possível garantir que existe uma subsequência de $\widetilde{u}_{k}$ que converge a uma aplicação pseudo-holomorfa $\widetilde{u}$ de $\mathbb{C} \backslash \Gamma$ em $\left(\mathbb{R} \times S^{3}, \widehat{J}\right)$. Todos os furos de $\widetilde{u}$ em $\Gamma$ são negativos (algo que já mencionamos e que será mostrado na próxima subseção). A cada um desses furos está associada uma órbita periódica de $X_{\lambda}$. Logo se o conjunto $\Gamma$ é não-vazio prova-se a existência de órbitas periódicas para o campo $X_{\lambda}$, ou em outras palavras prova-se a Conjectura de Weinstein para a estrutura de contato tight em $S^{3}$. A próxima proposição garante que $\Gamma$ é não vazio, e caracteriza $\Gamma$ no caso em que $\lambda$ é uma forma de contato induzida em $S^{3}$ via uma hipersuperfície convexa de $\mathbb{R}^{4}$ (segundo a construção no Capítulo 1 ).

Proposição 8. Para qualquer forma de contato $\lambda$ associada a $\left(S^{3}, \xi^{0}\right)$ temos $0 \in \Gamma$. Caso $\lambda$ seja induzida em $S^{3}$ via uma hipersuperfície convexa de $\mathbb{R}^{4}$ (ou, mais geralmente, $\lambda$ dinamicamente convexa) temos $\Gamma=\{0\}$.

Demonstração: Passo 1: mostrar que 0 é ponto de bubbling.

Tomemos $z_{0}$ com $\left|z_{0}\right|>1$. Pela proposição anterior temos que a sequência $a_{k}\left(z_{0}\right)$ é limitada. De $a_{k}(0) \rightarrow-\infty$, segue:

$$
\left|a_{k}\left(z_{0}\right)-a_{k}(0)\right| \rightarrow+\infty
$$

Ligando 0 e $z_{0}$ por qualquer caminho temos que as funções $\left|a_{k}^{\prime}\right|$ nesse caminho apresentam valores arbitrariamente grandes, quando $k \rightarrow+\infty$, em consequência do teorema do valor médio. Isso implica que sempre há um ponto de bubbling nesse caminho, pois a norma do gradiente de $\widetilde{u}_{k}$ é maior que $\left|a_{k}^{\prime}\right|$.

Agora $z_{0}$ não pode ser ponto de bubbling, como já observamos. Se 0 não fosse ponto de bubbling seria possível conectar 0 e $z_{0}$ por um caminho sem pontos de bubbling já que estes são sempre finitos. Mas acabamos de verificar que isso é impossível, o que garante a primeira parte do enunciado de nosso lema.

Passo 2: no caso $\lambda$ ser induzida por uma hipersuperfície convexa só pode haver um ponto de bubbling.

Pelo principal teorema do capítulo 2 vimos que todas as órbitas periódicas $X_{\lambda}$ tem índice de Conley-Zehnder maior ou igual a 3. Será demonstrado na próxima subseção que todos os pontos de bubbling de $\Gamma$ são furos negativos. Temos que em $\mathbb{C} \backslash \Gamma$ os mapas $\widetilde{u}_{k}$ convergem a uma 
curva pseudo-holomorfa $\widetilde{v}: \mathbb{C} \backslash \Gamma \rightarrow\left(\mathbb{R} \times S^{3}, \widehat{J}\right)$. É essencial para sabermos que esse limite tem imagem em $\left(\mathbb{R} \times S^{3}, \widehat{J}\right)$ a proposição anterior, que garante que os pontos $\widetilde{u}_{k}(z)$ ficam presos em conjuntos limitados do cobordismo para $|z|>1$. Do contrário, essa curva limite $\widetilde{v}$ poderia surgir na simplectização de $\left(S^{3}, \lambda\right)$ ou de $\left(S^{3}, \lambda_{E}\right)$.

Calculamos agora o índice de Fredholm de $\widetilde{v}$ :

$$
\operatorname{Fred}(\widetilde{v})=\mu_{C Z}\left(P_{0}\right)-\mu^{-}(\widetilde{v})-\chi\left(S^{2}\right)+1+\# \Gamma=2+\# \Gamma-\mu^{-}(\widetilde{v}) \leq 3+\# \Gamma-3 \Gamma=3-2 \# \Gamma,
$$

onde $\mu^{-}(\widetilde{v})$ é a soma dos índices de Conley-Zehnder dos furos negativos. Na desigualdade acima utilizamos que o índice de Conley-Zehnder de todas órbitas de $X_{\lambda}$ é maior ou igual a que 3, e na primeira igualdade usamos que todos os furos em $\Gamma$ são negativos.

$O$ fato do índice de Fredholm ser sempre não-negativo para uma escolha genérica de $\widehat{J}$ implica então que $\# \Gamma=1$. Isso mostra só pode haver um ponto de bubbling para o caso em que $X_{\lambda}$ só tem órbitas periódicas com índice de Conley-Zehnder maior ou igual a 3, que é o 0 pelo passo 1.

Como observamos anteriormente o fato de 0 ser ponto de bubbling para toda forma $\lambda$ implica que $X_{\lambda}$ sempre tem uma órbita periódica. Uma consequência desse fato é uma prova que níveis de energia starshaped em $\mathbb{R}^{4}$ sempre tem órbitas periódicas, um resultado seminal que Rabinowitz obteve por métodos variacionais $([23])$.

\subsubsection{O conjunto $\Gamma$}

Esta seção contém a prometida prova do resultado que mencionamos anteriormente à respeito do conjunto de furos $\Gamma$ que apareceram nas curvas pseudo-holomorfas $\widetilde{v}: \mathbb{C} \backslash \Gamma \rightarrow\left(\mathbb{R} \times S^{3}, J_{i}\right)$, onde $J_{i}=\widehat{J}$ ou $J_{i}=J_{\lambda}$.

Proposição 9. Seja $\widetilde{u}_{k} \in \mu$ uma sequência de planos pseudo-holomorfos e $\Gamma$ o conjunto dos pontos de bubbling dessa sequência. Suponha que ocorra uma das duas possibilidades:

a) existe $z_{0}$ com $z_{0} \in \mathbb{C} \backslash \Gamma$ e uma subsequência $\widetilde{u}_{l}$, tal que $a_{l}\left(z_{0}\right) \rightarrow+\infty$

b) existe $z_{0}$ com $z_{0} \in \mathbb{C} \backslash \Gamma$ e uma subsequência $\widetilde{u}_{l}$, tal que $a_{l}\left(z_{0}\right)$ fica preso em um conjunto compacto de $\mathbb{R} \times S^{3}$.

Então,

- no caso a) para toda $\widetilde{v}: \mathbb{C} \backslash \Gamma \rightarrow\left(\mathbb{R} \times S^{3}, J_{\lambda_{E}}\right)$ obtida como limite de uma subsequência $\widetilde{v}_{l}=\left(a_{l}(z)-a_{l}\left(z_{0}\right), u_{l}(z)\right)$, os pontos $\Gamma$ são furos negativos de $\widetilde{v}$

- no caso b) para toda $\widetilde{v}: \mathbb{C} \backslash \Gamma \rightarrow\left(\mathbb{R} \times S^{3}, \widehat{J}\right)$ que é limite de uma subsequência de $\widetilde{u}_{l}$ todos os furos do conjunto $\Gamma$ são furos negativos.

Demonstração: Caso a):

Em qualquer compacto contido em $\mathbb{C} \backslash \Gamma$ a norma do gradiente da sequência $\widetilde{v}_{l}$ é uniformemente limitada (pois os pontos de bubbling de $\widetilde{v}_{l}$ e $\widetilde{u}_{l}$ são os mesmos). Seja $K_{n}$ uma sequência de compactos encaixantes tal que $\bigcup K_{n}=\mathbb{C} \backslash \Gamma$. Para cada $K_{n}$ existe $l_{n}$ tal que $\widetilde{u}_{l}\left(K_{n}\right) \subset[3,+\infty]$ para todo $l>l_{n}$. Isso garante que o mapa $\widetilde{v}_{l_{n}}: K_{n} \rightarrow\left(\mathbb{R} \times S^{3}, J_{\lambda_{E}}\right)$ é pseudo-holomorfo.

Temos então uma sequência $\widetilde{v}_{l_{n}}: K_{n} \rightarrow\left(\mathbb{R} \times S^{3}, J_{\lambda_{E}}\right)$, com gradiente uniformemente limitado em compactos, $\widetilde{v}_{l_{n}}(0) \in\{0\} \times S^{3}$ e $\bigcup K_{n}=\mathbb{C} \backslash \Gamma$. Nessas condições a regularidade elíptica nos permite concluir que existe uma subsequência de $\widetilde{v}_{l_{n}}$ que converge a uma curva pseudo-holomorfa $\widetilde{v}: \mathbb{C} \backslash \Gamma \rightarrow\left(\mathbb{R} \times S^{3}, J_{\lambda_{E}}\right)$. Seja $\widetilde{v}$ uma dessas aplicações. Observamos inicialmente que $\widetilde{v}$ tem energia finita.

Suponha por absurdo que um dos furos $z_{j} \in \Gamma$ é um furo positivo. Então conhecemos o comportamento de $\widetilde{v}$ na vizinhança de $z_{j}$, em particular, tem de ocorrer: $\lim _{\epsilon \rightarrow 0} \min \left\{b(z) ;\left|z-z_{j}\right|=\right.$ $\epsilon\}=+\infty$ onde $b$ é a coordenada real de $\widetilde{v}$. Seja então $\epsilon_{0}>0$ e $10<N_{0}=\max \left\{b(z) ;\left|z-z_{j}\right|=\epsilon_{0}\right\}$. Tomemos $0<\epsilon_{1}<\epsilon_{0}$ tal que $\min \left\{b(z) ;\left|z-z_{j}\right|=\epsilon_{1}\right\}=N_{0}+10^{10}$. Como existe uma subsequência de $\widetilde{v}_{l_{n}}$ que converge à $\widetilde{v}$ é possível achar $l^{\prime}$ tal que: 


$$
\begin{aligned}
& \max \left\{b_{l^{\prime}}(z) ;\left|z-z_{j}\right|=\epsilon_{0}\right\} \leq N_{0}+1 \\
& \min \left\{b_{l^{\prime}}(z) ;\left|z-z_{j}\right|=\epsilon_{1}\right\} \geq N_{o}+10^{10}-1 .
\end{aligned}
$$

Lembramos que $\widetilde{u}_{l^{\prime}}(z)=\left(b_{l^{\prime}}(z)+a_{l^{\prime}}\left(z_{0}\right), v_{l^{\prime}}(z)\right)$. Existem duas possibilidades:

1) $b_{l^{\prime}}(z) \in[2,+\infty) ; \forall z \in \overline{B_{\epsilon_{0}}\left(z_{j}\right)}$

2) $b_{l^{\prime}}(z)<2$ para algum $z \in \overline{B_{\epsilon_{0}}\left(z_{j}\right)}$

Possibilidade 1)

Nesse caso $\widetilde{u}_{l^{\prime}}\left(\overline{B_{\epsilon_{0}}\left(z_{j}\right)}\right)$ é um disco pseudo-holomorfo em $\left([2,+\infty) \times S^{3}, \widehat{J}\right)$, mas nessa parte do cobordismo a estrutura quase-complexa $\widehat{J}$ coincide com $J_{\lambda_{E}}$, que é uma estrutura quasecomplexa de uma simplectização. Em estruturas quase-complexas de simplectizações, curvas pseudo-holomorfas tem de satisfazer o princípio do máximo, que diz que o máximo de $\left.a_{l^{\prime}}\right|_{B_{\epsilon_{0}}\left(z_{j}\right)}$ tem de estar no bordo de $\overline{B_{\epsilon_{0}}\left(z_{j}\right)}$. Nosso disco $\widetilde{u}_{l^{\prime}}\left(\overline{B_{\epsilon_{0}}\left(z_{j}\right)}\right)$ deve portanto satisfazer essa condição. Entretanto isso não ocorre pois o bordo de $\overline{B_{\epsilon_{0}}\left(z_{j}\right)}$ é o conjunto $\left\{z ;\left|z-z_{j}\right|=\epsilon_{0}\right\}$ e:

$$
\begin{gathered}
\max \left\{a_{l^{\prime}}(z) ;\left|z-z_{j}\right|=\epsilon_{0}\right\}=N_{0}+1+a_{l^{\prime}}\left(z_{0}\right), \\
\max \left\{a_{l^{\prime}}(z) ;\left|z-z_{j}\right| \leq \epsilon_{0}\right\}<\min \left\{a_{l^{\prime}}(z) ;\left|z-z_{j}\right|=\epsilon_{1}\right\}=N_{0}+10^{10}-1+a_{l^{\prime}}\left(z_{0}\right) .
\end{gathered}
$$

As duas equações acima implicam que o máximo de $\left.a_{l^{\prime}}\right|_{B_{\epsilon_{0}}\left(z_{j}\right)}$ não ocorre no bordo. Isso nos dá uma contradição na possibilidade 1), com o princípio do máximo.

2) Possibilidade 2)

Seja $V=\left\{z \in \overline{B_{\epsilon_{0}}\left(z_{j}\right)} ; a_{l^{\prime}}(z)<2\right\}$. Então $V$ é um aberto em $\overline{B_{\epsilon_{0}}\left(z_{j}\right)}$ e $\overline{B_{\epsilon_{0}}\left(z_{j}\right)} \backslash V$ é um compacto. Consideremos então a restrição $a_{l^{\prime}} \mid \overline{B_{\epsilon_{0}}\left(z_{j}\right) \backslash V}$, e por $\widetilde{u}_{l^{\prime}}\left(\overline{B_{\epsilon_{0}}\left(z_{j}\right)} \backslash V\right)$ ser um disco pseudoholomorfo furado com imagem contida em $\left([2,+\infty) \times S^{3}, \widehat{J}\right)$ deve valer o princípio do máximo para a restrição $a_{l^{\prime}} \mid \overline{B_{\epsilon_{0}}\left(z_{j}\right)} \backslash V$.

Mostraremos que isso não ocorre. Pela construção de $V$ temos que a fronteira $\partial\left(\overline{B_{\epsilon_{0}}\left(z_{j}\right)} \backslash V\right)$ é formada pelo conjunto $\left\{z ;\left|z-z_{j}\right|=\epsilon_{0}\right\}$ unido a um conjunto compacto $X$ que satisfaz $b_{l^{\prime}}(x)=$ $2 \forall x \in X$. Temos então:

$$
\max \left\{a_{l^{\prime}}(z) ; z \in \partial\left(\overline{B_{\epsilon_{0}}\left(z_{j}\right)} \backslash V\right)\right\}=\max \left\{N_{0}+1+a_{l^{\prime}}\left(z_{0}\right) ; 2\right\}=N_{0}+1+a_{l^{\prime}}\left(z_{0}\right),
$$

pois $N_{0}>10$ e $a_{l^{\prime}}\left(z_{0}\right)>2$.

Por outro lado como o conjunto $\left\{\left|z-z_{j}\right|=\epsilon_{1}\right\}$ está contido em $\overline{B_{\epsilon_{0}}\left(z_{j}\right)} \backslash V$ :

$$
\max \left\{a_{l^{\prime}}(z) ;\left|z-z_{j}\right| \leq \epsilon_{0}\right\}<\min \left\{a_{l^{\prime}}(z) ;\left|z-z_{j}\right|=\epsilon_{1}\right\}=N_{0}+10^{10}-1+a_{l^{\prime}}\left(z_{0}\right),
$$

e novamente ocorre uma violação do principio do máximo.

Concluímos então que a existência de um furo positivo $z_{j} \in \Gamma$ para $\widetilde{v}$ sempre implica numa violação do princípio do máximo no caso a) e portanto, numa contradição.

Fim do caso a).

Caso b)

No caso b) supomos a existência de um furo positivo $z_{j}$ em $\Gamma$ para $\widetilde{v}: \mathbb{C} \backslash \Gamma \rightarrow\left(\mathbb{R} \times S^{3}, \widehat{J}\right)$ que é limite de uma subsequência de $\widetilde{u}_{l}$. Então tem de ocorrer: $\lim _{\epsilon \rightarrow 0} \min \left\{b(z) ;\left|z-z_{j}\right|=\epsilon\right\}=+\infty$ onde $b$ é a coordenada real de $\widetilde{v}$. Isso torna possível que assim como no caso a), encontremos $0<\epsilon_{1}<\epsilon_{0}$ satisfazendo: 


$$
\begin{aligned}
\max \left\{b(z) ;\left|z-z_{j}\right|\right. & \left.=\epsilon_{0}\right\}=N_{0}>10 \\
\min \left\{b(z) ;\left|z-z_{j}\right|\right. & \left.=\epsilon_{1}\right\}=N_{0}+10^{10}
\end{aligned}
$$

Exigimos ainda a condição extra $\min \left\{b(z) ;\left|z-z_{j}\right|=\epsilon_{0}\right\} \geq 3$.

Devido à $\widetilde{v}: \mathbb{C} \backslash \Gamma \rightarrow\left(\mathbb{R} \times S^{3}, \widehat{J}\right)$ ser limite de uma subsequência de $\widetilde{u}_{l}$, é possível encontrar $l^{\prime}$ tal que $\widetilde{u}_{l^{\prime}}$ satisfaz:

$$
\begin{gathered}
\max \left\{a_{l^{\prime}}(z) ;\left|z-z_{j}\right|=\epsilon_{0}\right\} \leq N_{0}+1, \\
\min \left\{a_{l^{\prime}}(z) ;\left|z-z_{j}\right|=\epsilon_{1}\right\} \geq N_{0}+10^{10}-1, \\
\min \left\{a_{l^{\prime}}(z) ;\left|z-z_{j}\right|=\epsilon_{0}\right\} \geq 2 .
\end{gathered}
$$

A partir desse ponto a demonstração segue os mesmos passos do caso a) dividindo-se em duas possibilidades:

1) $b_{l^{\prime}}(z) \in[2,+\infty) ; \forall z \in \overline{B_{\epsilon_{0}}\left(z_{j}\right)}$

2) $b_{l^{\prime}}(z)<2$ para algum $z \in \overline{B_{\epsilon_{0}}\left(z_{j}\right)}$

A argumentação para obter uma contradição com o principio do máximo é idêntica e por isso não a repetimos, concluindo assim a demostração da proposição.

\subsection{A árvore de bubbling}

Supondo que $\lambda$ é dinamicamente convexa, obtivemos através do limite da sequência $\widetilde{u}_{k} \in \mu$ duas curvas pseudo-holomorfas:

1) um cilindro $\widetilde{v}: \mathbb{C} \backslash\{0\} \rightarrow\left(\mathbb{R} \times S^{3}, \widehat{J}\right)$ no cobordismo que é assintótico à órbita periódica $P_{0}$ quando a norma de $z \in \mathbb{C}$ vai para $+\infty$ e é assintótico quando $|z| \rightarrow 0$ a uma órbita periódica $P^{-}$ de $X_{\lambda}$.

2) um plano de energia finita $\widetilde{u}: \mathbb{C} \rightarrow\left(\mathbb{R} \times S^{3}, J_{\lambda}\right)$ que é assintótico a uma órbita periódica $P^{+}$ de $X_{\lambda}$.

Uma pergunta natural é: qual a relação entre as órbitas $P^{-}$e $P^{+}$? O que ocorre é que $P^{-}=P^{+}$. Iremos agora dar uma ideia da demonstração desse fato. Para isso iremos primeiramente analisar o caso geral de $\lambda$ uma forma de contato tight em $S^{3}$.

No caso geral o conjunto $\Gamma$ dos pontos de bubbling da sequência $\widetilde{u}_{k}$ pode conter outros pontos além de 0 . Nesse caso obtemos inicialmente $\widetilde{v}: \mathbb{C} \backslash \Gamma \rightarrow\left(\mathbb{R} \times S^{3}, \widehat{J}\right)$. Seja $\Gamma=\left\{z_{1}, \ldots, z_{j}\right\}$. Todos esses furos de $\widetilde{v}$ são furos negativos, e portanto $\widetilde{v}$ é assintótico em cada $z_{i} \in \Gamma$ a uma órbita periódica $P_{i}$ de $X_{\lambda}$. Usando ao redor do ponto de bubbling uma técnica de re-escala chamada "soft rescaling" (introduzida em [11]) é possível associar a cada ponto $z_{i} \in \Gamma$ um conjunto finito $\Gamma_{i}$ e uma curva pseudo holomorfa $\widetilde{v}_{i}: \mathbb{C} \backslash \Gamma_{i} \rightarrow\left(\mathbb{R} \times S^{3}, J_{\lambda}\right)$ tal que:

- quando $|z| \rightarrow+\infty$ temos $\widetilde{v}_{i}$ é assintótica à $P_{i}$ (ver [12]).

- o conjunto $\Gamma_{i}$ é composto de furos negativos de $\widetilde{v}_{i}$.

- $\widetilde{v}_{i}$ não é um cilindro sobre a órbita periódica $P_{i}$.

Repetindo o "soft-rescaling" para cada elemento de $\Gamma_{i}$ obteríamos então outras curvas pseudoholomorfas associadas com as três propriedades acima. Podemos então prosseguir aplicando esse raciocínio de "soft rescalling"seguidamente. Entretanto, esse processo só é feito um número finito de vezes, pois há uma limitação uniforme de energia para as curvas geradas. Após um número finito de aplicações deste processo o "soft-rescaling" gera então um plano pseudo-holomorfo de energia finita que é um dos planos $\widetilde{u}_{i}$ gerados pelo processo de reescala que apresentamos na proposição 6 aplicado ao ponto de bubbling $z_{i}$. O resultado é a chamada árvore de bubbling. Essa árvore de bubbling é chamada de limite da sequência $\widetilde{u}_{k}$ na chamada "Symplectic Field Theory" (SFT). Nesta teoria é definida uma compactificação para espaços de curvas pseudo-holomorfas de energia finita, 
e nessa compactificação o limite de uma sequência de curvas pseudo-holomorfas é um conjunto de curvas pseudo-holomorfas de energia finita (a curva quebra-se nesse conjunto de curvas; ver [4], [1]).

Voltamos agora ao caso em que $\lambda$ é dinamicamente convexa. Nesse caso $\Gamma=\{0\}$, e $\widetilde{v}$ é assintótico quando $|z| \rightarrow 0$ a uma órbita periódica de $X_{\lambda}$ que denotaremos $P_{1}$. Vimos anteriormente que para que o indíce de Fredholm de $\widetilde{v}$ seja não negativo é necessário que a órbita $P_{1}$ tenha índice de ConleyZehnder igual à 3 . Afirmamos então que a curva $\widetilde{v}_{0}$ produzida pelo "soft-rescaling" na origem é um plano de energia finita. Seja $\Gamma_{0}$ o conjunto de furos de $\widetilde{v}_{0}$. Então, usando a fórmula dada em [16] calculamos o índice de Fredholm de $\widetilde{v}_{0}$ :

$\operatorname{Fred}\left(\widetilde{v}_{0}\right)=\mu_{C Z}\left(P_{1}\right)-\mu^{-}(\widetilde{v})-\chi\left(S^{2}\right)+1+\# \Gamma_{0}=2+\# \Gamma_{0}-\mu^{-}(\widetilde{v}) \leq 2+\# \Gamma_{0}-3 \Gamma_{0}=2-2 \# \Gamma_{0}$,

onde $\mu^{-}\left(\widetilde{v}_{0}\right)$ é a soma dos índices de Conley-Zehnder dos furos negativos. Na desigualdade acima foi utilizado que o índice de Conley-Zehnder de todas órbitas de $X_{\lambda}$ é maior ou igual à 3 , e que todos os furos em $\Gamma_{0}$ são negativos.

Para que o índice de Fredholm seja não-negativo devemos ter $\# \Gamma_{0} \leq 1$. Por absurdo suponha $\Gamma \neq \emptyset$. Podemos considerar sem perda de generalidade que $\Gamma_{0}=\{0\}$. Nesse caso teríamos Fred $\left(\widetilde{v}_{0}\right)=0$. Entretanto, como $\widetilde{v}_{0}$ é um cilindro pseudo holomorfo em uma simplectização, que não é um cilindro sobre uma órbita periódica, tem de valer: $\operatorname{Fred}\left(\widetilde{v}_{0}\right) \geq 1$. Isso ocorre por que dado $\widetilde{v}_{0}: \mathbb{C} \backslash\{0\} \rightarrow\left(\mathbb{R} \times S^{3}, J_{\lambda}\right)$ (que não é um cilindro sobre uma órbita periódica) podemos gerar uma família unidimensional de cilindros pseudo-holomorfos, definindo para cada $a \in \mathbb{R}$ :

$$
\widetilde{v}_{0}^{a}(z)=\left(b_{0}(z)+a, v_{0}(z)\right) .
$$

Temos que $\widetilde{v}_{0}^{a}$ é pseudo-holomorfa, porque a estrtura quase-complexa $J_{\lambda}$ em $\mathbb{R} \times S^{3}$ é invariante por translações em $\mathbb{R}$. No caso em que $\widetilde{v}_{0}$ não é um cilindro sobre uma órbita periódica, temos que a imagem da curva $\widetilde{v}_{0}^{a}$ é diferente da imagem de $\widetilde{v}_{0}$ (basta tomar um número real $a_{0}$ tal que a imagem de $\widetilde{v}_{0}^{a}$ intersecção com $\left\{-a_{0}\right\} \times S^{3}$ não seja uma das órbitas periódicas limites e considerar $\left.\widetilde{v}_{0}^{a_{0}}\right)$. Logo ocorre que $\operatorname{Fred}\left(\widetilde{v}_{0}\right) \geq 1$ o que implica que $\Gamma_{0}=\emptyset$.

Sendo assim a curva $\widetilde{v}_{0}$ gerada pelo "soft-rescaling"é um plano pseudo-holomorfo assintótico à órbita $P^{-}$quando $|z| \rightarrow+\infty$. Mas como esse é um plano gerado na última iteração do processo de re-escala, ele deve coincidir com o plano $\widetilde{u}$ produzido pela análise de bubbling usual no ponto 0 . Isso prova a igualdade $P^{-}=P^{+}$.

\subsection{A decomposição em openbook para níveis de energia convexos}

Nessa seção iremos estudar o espaço $\Theta$ de planos pseudo-holomorfos assintóticos à órbita $P$ do campo de Reeb $X_{\lambda}$ onde $\lambda$ é uma forma dinamicamente convexa. Usando o plano pseudo-holomorfo $\widetilde{u}$ construído anteriormente, iremos ver que a componente conexa de $\Theta$ que contém $\widetilde{u}$ dá origem a uma decomposição em openbook de $S^{3}$ na qual as páginas são projeções em $S^{3}$ de planos pseudoholomorfos assintóticos à $P$. Esse resultado usa a chamada compacidade SFT, que mencionamos na seção anterior, e que iremos assumir sem demonstrações, para apresentar o seguinte:

Teorema 6. Seja $\lambda$ uma forma dinamicamente convexa tight em $S^{3}$ e $\widetilde{u}$ um plano de energia finita tal que $u(\mathbb{C})$ é um mergulho transversal à $X_{\lambda}$ e assintótico a uma órbita periódica $P$ com índice de Conley-Zehnder 3 e período T. Então existe uma decomposição em openbook de $S^{3}$ dada por uma aplicação diferenciável $\Psi: S^{1} \times \mathbb{C} \rightarrow S^{3} \backslash P$ que satisfaz: 


$$
\begin{aligned}
\Psi(\theta, \cdot): \mathbb{C} \rightarrow & S^{3} \text { é um disco mergulhado transversal à } X_{\lambda}, \\
\forall \theta \in S^{1} ; \exists \widetilde{u}_{\theta}: \mathbb{C} \rightarrow & \left(\mathbb{R} \times S^{3}, J_{\lambda}\right) \text { pseudo-holomorfo assintótico à } P \text { tal que, } \\
& u_{\theta}(\cdot)=\Psi(\theta, \cdot) \text { e } u(\cdot)=\Psi(1, \cdot) .
\end{aligned}
$$

Idéia da demonstração: A prova é baseada na compactificação de espaços de planos pseudoholomorfos feita em $([4,[13])$. Inicialmente notamos que pela teoria de Fredholm desenvolvida em [16] temos que $\widetilde{u}$ pertence a uma família $\Theta$ a 2 parâmetros de planos pseudo-holomorfos assintóticos à $P$. Um desses parâmetros é dado pela invariância da estrutura quase complexa $J_{\lambda}$ em relação à coordenada real. Para estudar essa família fazemos as seguintes normalizações para definir a família $\Theta^{\prime}$ :

$$
\begin{array}{r}
\widetilde{v}=(b, v) \in \Theta, \\
\int_{\mathbb{D}} \widetilde{v}^{*} d \lambda=T-\frac{\gamma}{2},
\end{array}
$$

onde $\gamma$ é o ínfimo da diferença $T^{\prime}-T, T^{\prime}$ sendo período de uma órbita periódica de $X_{\lambda}$ e $T^{\prime}<T$. O fato de $\lambda$ ser não-degenerada implica $\gamma \neq 0$.

Tomamos então uma sequência $\widetilde{v}_{k}$ de elementos em $\Theta^{\prime}$ e utilizando o "soft-rescaling" estudamos a curva pseudo-holomorfa quebrada que é o limite da sequência $\widetilde{v}_{k}$ no sentido SFT. Sabemos que no primeiro passo, geramos uma curva pseudo-holomorfa $\widetilde{v}: \mathbb{C} \backslash \Gamma \rightarrow\left(\mathbb{R} \times S^{3}, J_{\lambda}\right)$ onde $\Gamma$ é um conjunto finito. Como observamos na seção anterior $\widetilde{v}$ é assintótico à $P$ quando $|z| \rightarrow+\infty$, todos os pontos de $\Gamma$ são furos negativos e $\widetilde{v}$ não é um cilindro sobre uma órbita periódica. Usando a teoria de Fredholm e o fato de $\lambda$ ser dinamicamente convexa calculamos o índice de Fredholm $\widetilde{v}$ :

$$
\operatorname{Fred}(\widetilde{v})=\mu_{C Z}(P)-\mu^{-}(\widetilde{v})-\chi\left(S^{2}\right)+1+\# \Gamma=2+\# \Gamma-\mu^{-}(\widetilde{v}) \leq 2+\# \Gamma-3 \Gamma=2-2 \# \Gamma .
$$

Como Fred $(\widetilde{v}) \geq 0$ temos necessariamente $\# \Gamma \leq 1$. Suponha que $\# \Gamma=1$, e portanto Fred $(\widetilde{v})=$ 0 . Nesse caso $\widetilde{v}$ é um cilindro pseudo-holomorfo que não é um cilindro sobre uma órbita periódica de $X_{\lambda}$. Como vimos na seção anterior, pelo fato de $J_{\lambda}$ ser invariante pela translação na coordenada real, esse cilindro pertence a uma família 1 parâmetro de cilindros pseudo-holmorfos em $\left(\mathbb{R} \times S^{3}, J_{\lambda}\right)$. Mas isso implicaria $\operatorname{Fred}(\widetilde{v})>0$, o que contradiz a fórmula acima. Logo $\widetilde{v}$ é também um plano pseudo-holomorfo de $\Theta$. Isso prova a compacidade no sentido SFT da família $\Theta$.

Por um argumento idêntico ao apresentado no Apêndice A sabemos que todos os elementos de $\Theta$ são mergulhos e suas projeções em $S^{3}$ são transversais a $X_{\lambda}$. Considere a seguinte família de planos pseudo-holomorfos:

$$
\begin{array}{r}
\widetilde{v}=(b, v) \in \Theta, \\
\int_{\mathbb{D}} \widetilde{v}^{*} d \lambda=T-\frac{\gamma}{2}, \\
\min \{b(\mathbb{C})\}=0 .
\end{array}
$$

Parametrizamos então essa família do seguinte modo: $\widetilde{v}_{t}$ para $t \in \mathbb{R}$ satisfaz $\operatorname{vol}\left(v_{t}(\mathbb{C}) ; t \in\right.$ $[a, b])=T(b-a)$ (estamos usando o volume definido em $S^{3}$ por $\lambda \wedge d \lambda$ e a teoria de Fredholm nos garante que essa família quando projetada em $S^{3}$ é unidimensional). O argumento de compacidade esboçado acima implica que a parametrização definida acima para todo $t \in \mathbb{R}$. Usando as técnicas expostas no Apêndice A sabemos que se dois planos dessa família se intersectam eles são necessariamente iguais. A normalização do volume dada acima implica então que existe $t_{0}$ tal que $\widetilde{v}_{t+t_{0}}=\widetilde{v}_{t} ; \forall t \in \mathbb{R}$. Essa parametrização define a decomposição em openbook do teorema. 


\section{Capítulo 4}

\section{Uma seção global em um nível de energia convexo}

\subsection{Existência da seção global}

Nesse capítulo, usaremos o plano de energia finita construído no capítulo anterior para construir uma seção global para o fluxo de Reeb. Continuamos com a hipótese de que todas as órbitas do fluxo tenham índice de Conley-Zehnder maior ou igual a 3. A seção global será a projeção $u(\mathbb{C})$ em $S^{3}$ do plano de energia finita $\widetilde{u}(\mathbb{C})$ construído no capítulo anterior. Obteremos também importantes consequências dinâmicas a partir dessa seção global.

Mencionamos inicialmente um resultado que por motivos técnicos será demonstrado no apêndice.

Teorema 7. Seja $\widetilde{u}$ o plano de energia finita na simplectização de $\left(S^{3}, \lambda\right)$ construído no capítulo 3. Então u(C) é sempre transversal ao campo de Reeb $X_{\lambda}$.

Usando esse resultado demonstraremos agora o seguinte teorema:

Teorema 8. Se $\lambda$ é dinamicamente convexa, $D:=u(\mathbb{C})$ é uma seção global para o fluxo de Reeb de $\lambda$.

Demonstração: Seja $P$ a órbita periódica limite do plano $\widetilde{u}$. Para que $u(\mathbb{C})$ seja uma seção global para o fluxo de Reeb, é necessário e suficiente, que para todos os pontos $x \in S^{3}$ que não estejam na órbita $P$, as trajetórias de fluxo $\phi^{t}(x)$ intersectem $u(\mathbb{C})$ para $t$ negativo e positivo. Isso em conjunto com a transversalidade de $X_{\lambda}$ e $u(\mathbb{C})$, implicam que o conjunto $\left\{t \in \mathbb{R} ; \phi^{t}(x) \in u(\mathbb{C})\right\}$ é discreto e ilimitado tanto superiormente quanto inferiormente.

Seja então $x \in S^{3} \backslash P$ e considere $\phi^{t}(x)$. Existem duas possibilidades:

a) $\omega^{+}(x) \cap P=\emptyset$.

b) $\omega^{+}(x) \cap P \neq \emptyset$.

Trataremos em separado esses dois casos.

Caso a)

Neste caso, existe uma $\epsilon$-vizinhança $V_{\epsilon}$ da órbita $P$ em $S^{3}$ na qual a trajetória $\phi^{t}(x)$ nunca entra, ou seja, $\left\{\phi^{t}(x) ; t \in \mathbb{R}\right\} \cap V_{\epsilon}=\emptyset$.

Usando a decomposição em openbook $\Phi$ mencionada no capítulo anterior, da qual $u(\mathbb{C})$ é uma página, podemos associar a cada página da decomposição um elemento de $S^{1}$ de maneira biunívoca. Mais ainda, como $\Phi: S^{1} \times \mathbb{C} \rightarrow S^{3} \backslash P$ é diferenciável, a aplicação $f=\pi_{1} \circ \Phi^{-1}: S^{3} \backslash V_{\epsilon} \rightarrow S^{1}$ é diferenciável (onde $\pi_{1}: S^{1} \times \mathbb{C} \rightarrow S^{1}$ é a projeção na primeira coordenada). Podemos além disso garantir que $f$ não tem pontos singulares (isso depende apenas da construção de $\Phi$ ). Usaremos em certos passos o levantamento $\widetilde{f}: S^{3} \backslash V_{\epsilon} \rightarrow \mathbb{R}$ de $f$ (esse levantamento é construído usando o recobrimento usual $\pi^{\prime}$ de $S^{1}=\mathbb{R} / \mathbb{Z}$ ). Observamos que, munindo $S^{3}$ de uma métrica Riemanniana, a norma do gradiente de $\widetilde{f}$ é maior que um certo $\delta>0$, pois $\widetilde{f}$ não tem pontos singulares e tem domínio compacto. 
Tomando $\phi^{t}(x)$ a trajetória passando por $x$ definimos:

$$
g(t)=\tilde{f} \circ \phi^{t}(x): \mathbb{R} \rightarrow \mathbb{R} .
$$

A derivada de $g$ é dada por:

$$
g^{\prime}(t)=d \widetilde{f}\left(\phi^{t}(x)\right)\left(X_{\lambda}\left(\phi^{t}(x)\right) \neq 0,\right.
$$

onde na desigualdade estamos usando o fato de que $\tilde{f}$ não tem pontos singulares, e que o campo de Reeb $X_{\lambda}$ é sempre transversal às páginas do openbook, que são as superfícies de nível de $\widetilde{f}$. Mais ainda, como $\mid d \widetilde{f}(x)\left(X_{\lambda}(x) \mid\right.$ é uma função contínua não-nula definida em um domínio compacto $S^{3} \backslash V_{\epsilon}$, existe $\epsilon>0$ tal que $\mid d \widetilde{f}(x)\left(X_{\lambda}(x)>\epsilon \mid\right.$. Temos portanto:

$$
\left|g^{\prime}(t)\right|=\mid d \widetilde{f}\left(\phi^{t}(x)\right)\left(X_{\lambda}\left(\phi^{T} t(x)\right) \mid>\epsilon, \forall t \in \mathbb{R}^{+} .\right.
$$

Iremos supor sem perda de generalidade que $g^{\prime}(t): \mathbb{R}^{+} \rightarrow \mathbb{R}$ é positiva. O fato de $g$ ter sua derivada cotada inferiormente por um número positivo, implica que $g$ tem uma taxa de crescimento mínima positiva, e que $g\left(\mathbb{R}^{+}\right)$é um aberto ilimitado superiormente, ou seja é da forma $[\alpha,+\infty]$. Fazendo agora $\pi^{\prime} \circ g$ temos a aplicação que a cada elemento da trajetória de fluxo associa uma página do openbook. $g\left(\mathbb{R}^{+}\right)=[\alpha,+\infty]$, força a aplicação $\pi^{\prime} \circ g: \mathbb{R}^{+} \rightarrow S^{1}$ a ser sobrejetora. Isso implica que $\phi^{t}(x)$ intersecta todas as superfícies de nível de $f$ para tempos positivos e portanto, intersecta nossa página $u(\widetilde{C})$ para tempos positivos.

Isso finaliza a demonstração do caso a)

Caso b)

Neste caso, dado $\epsilon>0$, temos que $\phi^{t}(x)$ fica na vizinhança $V_{\epsilon}$ para intervalos arbitrariamente grandes de tempo. Logo iremos começar nossa análise fazendo uma descrição do campo $X_{\lambda}$ em vizinhanças da órbita $P$.

Em [14] é exibida uma forma normal para formas de contato próximas à uma órbita periódica devida a Martinet, e usando essa forma normal é provada uma fórmula assintótica para semicilindros pseudo-holomorfos que são assintóticos a essa órbita periódica. Essa fórmula assintótica nos dá uma descrição das curvas pseudo-holomorfas quando estamos na vizinhança de um de seus furos. Iremos usar essa análise para obtermos informações do comportamento de $u(\mathbb{C})$ em uma vizinhança suficientemente pequena de $P$.

Inicialmente damos a descrição da forma de contato $\lambda$ na vizinhança de $P$. Existe uma vizinhança tubular $V$ de $P$, na qual é possível construir um difeomorfismo $\psi: S^{1} \times \mathbb{D} \rightarrow V$ e uma função $h: S^{1} \times \mathbb{D} \rightarrow \mathbb{R}$ tais que:

$$
\begin{aligned}
& \psi(\theta, 0)=P(\theta), \\
& \psi^{*} \lambda=h \lambda_{0}, \\
& h(\theta, 0)=T, \\
& d h(\theta, 0)=0,
\end{aligned}
$$

onde $T$ é o período mínimo de $P, \lambda_{0}=d \theta+\frac{x d y-y d x}{2}$ para $\theta \in S^{1}$. Considerando o recobrimento natural de $\mathbb{R} \times \mathbb{D}$ em $S^{1} \times \mathbb{D}$, podemos considerar a coordenada $\theta$ como pertencente a $\mathbb{R}$. Esse recobrimento nos permite levantar o mapa $\psi$, para gerar um recobrimento $\widetilde{\psi}: \mathbb{R} \times \mathbb{D} \rightarrow V$. É nesse sistema de coordenadas que exibiremos a fórmula assintótica para $\widetilde{u}$, que é:

$$
\begin{gathered}
\widetilde{u}\left(e^{2 \pi(s+i t)}\right)=\widetilde{\psi}\left(T s+c+r_{1}(s, t), T t+d+r_{2}(s, t), z(s, t)\right) \in \mathbb{R} \times \mathbb{R} \times \mathbb{D}, \\
z(s, t)=e^{\int_{s_{0}}^{s} \gamma(\sigma) d \sigma}\left(e(t)+r_{3}(s, t)\right),
\end{gathered}
$$

onde existem constantes $d>0$ e $M_{\alpha}>0$ tais que para todo $\alpha$ um multi-índice de elementos de $\mathbb{N}$ : 


$$
\begin{gathered}
\left|\partial^{\alpha} r_{j}(s, t)\right| \leq M_{\alpha} e^{-d s} j=1 ; 2, \\
\left|\partial^{\alpha} r_{3}(s, t)\right| \rightarrow 0 \text { uniformemente em } t \text { quando } s \rightarrow+\infty,
\end{gathered}
$$

e $e(t)$ é um autovetor do operador auto-adjunto $L_{P}$ (descrito no capítulo 2 e associado ao fluxo linearizado de $P(t)$ ) associado a um autovalor negativo $\gamma$. Por último temos que a função $\gamma(s) \rightarrow \gamma$ quando $s \rightarrow+\infty$. Para descrever o "giro" do fluxo de Reeb em relação a $u(\mathbb{C})$ próximo a $P$, vamos descrever primeiro o "giro" do fluxo linearizado em relação a um campo de vetores sobre a órbita $P$ que é tangente à superfície com bordo $u(\mathbb{C}) \cup P$, que é compatificação diferenciável de $u(\mathbb{C})$ em $S^{3}$. Esse campo é justamente $e(t)$. Para vermos isso notamos que em [14] é provado que a constante $d$ acima pode ser escolhida de forma à valer:

$$
\lim _{s \rightarrow+\infty} \frac{e^{-d s}}{e^{\int_{s_{0}}^{s} \gamma(\sigma) d \sigma}}=0
$$

O campo que desejamos estudar é: $\partial_{s}\left(T t+d+r_{2}(s, t), z(s, t)\right)=\left(\partial_{s} r_{2}(s, t), \partial_{s}\left(e^{\int_{s_{0}}^{s} \gamma(\sigma) d \sigma}(e(t)+\right.\right.$ $\left.\left.r_{3}(s, t)\right)\right)$. Observando que $\partial_{s}\left(e^{\int_{s_{0}}^{s} \gamma(\sigma) d \sigma}\right)=\gamma e^{\int_{s_{0}}^{s} \gamma(\sigma) d \sigma}$ temos:

$$
\partial_{s} z(s, t)=\gamma e^{\int_{s_{0}}^{s} \gamma(\sigma) d \sigma}\left(e(t)+r_{3}(s, t)\right)+e^{\int_{s_{0}}^{s} \gamma(\sigma) d \sigma}\left(\partial_{s} r_{3}(s, t)\right) .
$$

De 4.7 temos:

$$
\left.\frac{\partial_{s}\left(T t+d+r_{2}(s, t), z(s, t)\right)}{\gamma e^{\int_{s_{0}}^{s} \gamma(\sigma) d \sigma}}=\left(r_{4}(s, t), e(t)+r_{3}(s, t)+\frac{\partial_{s} r_{3}(s, t)}{\gamma}\right)\right) .
$$

Usando 4.6 temos:

$$
\left|r_{4}(s, t)\right|=\frac{\left|\partial_{s} r_{2}(s, t)\right|}{e^{\int_{s_{0}}^{s} \gamma(\sigma) d \sigma}} \leq M \frac{e^{-d s}}{e^{\int_{s_{0}}^{s} \gamma(\sigma) d \sigma}} \rightarrow 0
$$

Essa última estimativa nos dá que :

$$
\lim _{s \rightarrow+\infty} \frac{\partial_{s}\left(T t+d+r_{2}(s, t), z(s, t)\right)}{\gamma e^{\int_{s_{0}}^{s} \gamma(\sigma) d \sigma}}=e(t),
$$

ou seja, obtemos que se considerarmos $e(t)$ como um campo sobre a órbita $P$, ele é o limite de um múltiplo de um dos campos coordenados (na direção $\partial_{s}$ ) definido pelo sistema de coordenadas $u\left(e^{2 \pi(s+i t)}\right)$ no semi-cilindro. Logo se considerarmos o semi-cilindro $u\left(e^{2 \pi(s+i t)}\right)$, como uma variação de $P(t)$ na direção $\partial_{s}$ o campo que gera essa variação é justamente o campo que é limite de um múltiplo de $\partial_{s}$, ou seja $e(t)$.

O "giro" do fluxo linearizado em relação a $e(t)$ é descrito pelo seguinte:

Lema 7. Seja $z(t)$ uma solução não nula da equação $\dot{z}(t)=j S(t) z(t)$ onde $S$ é o caminho de matrizes simétricas associado ao fluxo linearizado em $P$. E seja e $(t)$ uma autofunção associada a um autovalor negativo de $L_{S}$. Então, $\exists \tilde{T}>0$ tal que:

$$
\left|\arg \left(z \bar{e}\left(t_{1}\right)\right)-\arg \left(z \bar{e}\left(t_{0}\right)\right)\right| \geq 3 \pi \text { se }\left|t_{1}-t_{0}\right|>\tilde{T},
$$

onde arg é um argumento contínuo em $\mathbb{R}^{2}$. 
Iremos adiar a prova do lema para não interromper nosso raciocínio e mostrar como ele pode ser usado para finalizar a demonstração do caso b).

Seja $\phi^{t}(x)$ uma solução do fluxo de Reeb próxima à $P$. Tomemos $(0, z) \in S^{1} \times \mathbb{D}$. Como o fluxo linearizado nos dá uma aproximação em primeira ordem do fluxo perto da órbita periódica temos:

$$
\phi^{t}(x)=\left(\phi^{t}(0), D \phi^{t}\left(\phi^{t}(0)\right) z+o\left(|z|^{2}\right)\right)=\left(t, D \phi^{t}\left(\phi^{t}(0)\right) z+o\left(|z|^{2}\right)\right),
$$

onde $o\left(|z|^{2}\right)$ é um termo de ordem dois em relação à $|z|$. Assim dado $\epsilon=\frac{\pi}{6}$, se $|z|$ for suficientemente pequeno o giro de $\phi^{t}(x)$ em relação ao campo $e(t)$ sobre $P(t)$ fica $\epsilon$-próximo do giro de $\left.D \phi^{t}(0)\right) z$ no intervalo $[0, \tilde{T}]$. Como o giro de $\left.D \phi^{t}(0)\right) z$ é maior ou igual à $3 \pi$ temos que o giro de $\phi^{t}(x)$ é maior que $\frac{5}{2} \pi$.

Mas como vimos anteriormente a superfície $u(\mathbb{C})$ próxima a $P$ pode ser vista como uma variação da curva $P(t)$ na direção do campo $e(t)$. Isso significa que tomando uma vizinhança tubular suficientemente pequena de $P$ temos que a superfície $u(\mathbb{C})$ tem para cada ponto $\theta$ de $S^{1}$ uma curva simples $\beta_{\theta}$ em $\{\theta\} \times \mathbb{D}$ ligando $(\theta, 0)$ a um ponto $\left(\theta, z_{\theta}\right)$ do bordo da vizinhança tubular; e essas curvas são tangentes a $e(\theta)$ em $S^{1}$. Para $|z|$ suficientemente pequena o giro de $D \phi^{t}(0) z$ em relação às curvas $\beta_{\theta}$ no intervalo $[0, \tilde{T}]$ é maior que $\frac{5}{2} \pi>2 \pi$. Isso implica que em algum $t \in[0, \tilde{T}]$ tem de haver uma intersecção entre $\left.\left(t, D \phi^{t}(0)\right) z\right)$ e uma curva $\left(t, \beta_{t}\right)$. Isso também garante que uma órbita suficientemente próxima de $P$ intersecta a curva pseudo-holomorfa $u(\mathbb{C})$. Podemos garantir que essa intersecção é transversal, pois o campo de Reeb $X_{\lambda}$ é transversal a $u(\mathbb{C})$. Decorre que as trajetórias sempre intersectam o disco $u(\mathbb{C})$ também no caso b).

O caso a) e b) garantem que todas as órbitas em $S^{3} \backslash P$ intersectam o dico $D$ para tempos positivos. Fazendo uma análise idêntica mas substituindo o $\omega^{+}$pelo conjunto $\alpha^{-}$, estabelece-se a mesma intersecção para tempos negativos. Isso termina a demonstração do teorema.

Demonstraremos agora o lema auxiliar que utilizamos.

Demonstração do lema:

Caso em que a órbita $P$ é não degenerada:

Chamemos $A(t)$ o caminho de matrizes simpléticas que representa o fluxo linearizado com relação à base da estrutura de contato sobre $P$ que contruímos na trivialização de $\xi^{0}$ exibida no Capítulo 1. Então $A(t)$ é solução da equação diferencial:

$$
\dot{A}(t)=J S(t) A(t) .
$$

Como $\mu_{C Z}(P)=3$ temos duas possibilidades para os autovalores de $A(T)$ :

1) os autovalores de $A(T)$ são $\lambda$ e $\bar{\lambda}$ ambos em $S^{1}$

2) os autovalores de $A(T)$ são $\lambda$ e $\frac{1}{\lambda}$ ambos negativos

Caso 1)

Nesse caso $A(T)$ é conjugada à uma matriz do tipo $\left[\begin{array}{rr}\cos (\rho) & -\sin (\rho) \\ \sin (\rho) & \cos (\rho)\end{array}\right]$, onde tomamos $\rho$ em $[0,2 \pi]$. Como é possível transformar $A(T)$ em $\left[\begin{array}{rr}\cos (\rho) & -\sin (\rho) \\ \sin (\rho) & \cos (\rho)\end{array}\right]$ via conjugação com uma matriz simplética $B$, podemos então fazer a conjugação $B A(t) B^{-1}$ que não muda a classe de homotopia do caminho $A(t)$ em $\Sigma(1)$. Como essa conjugação é apenas uma mudança de coordenadas de $\xi$ sobre $P$, ela não altera o giro do fluxo linearizado em relação à $e(t)$, e por isso não há perda de generalidade em considerarmos que:

$$
A(T)=\left[\begin{array}{rr}
\cos (\rho) & -\sin (\rho) \\
\sin (\rho) & \cos (\rho)
\end{array}\right]
$$

Sabemos que $z(t)$ por ser uma trajetória do fluxo linearizado é da forma $z(t)=A(t) z(0)$. Lembrando a definição geométrica do índice de Conley-Zehnder temos que se para cada $v \in \mathbb{R}^{2}$ considerarmos a curva $A(t) v$ e definirmos $\triangle(v)$ como sendo a variação de argumento dessa curva 
entre 0 e $T$, temos um intervalo $I(A)=\left\{\triangle(v), v \in \mathbb{R}^{2}\right.$ e $\left.v \neq 0\right\}$. Como $\mu_{C Z}(P)=3$ temos $I(A) \subset(1,2)$, o que implica que a variação de argumento de $z(t)$ entre $[0, T]$ é maior que $2 \pi$. Como $A(T)$ é uma rotação de ângulo $\rho \in(0,2 \pi)$ temos exatamente:

$$
\arg (z(T)-\arg z(0))=2 \pi+\rho .
$$

Lembramos agora que $e(t)$ é uma autofunção do operador $L_{S}$ (nesse caso como $P$ tem período $T$ temos que considerar $S^{1}=\mathbb{R} / T \mathbb{Z}$ na definição deste operador). Essa auto-função está associada a um autovalor negativo. Lembramos agora a segunda fórmula que apresentamos para calcular o índice generalizado no caso bi-dimensional.

$$
\tilde{\mu}_{C Z}^{1}(S)=2 \alpha(S)+p(S),
$$

onde $\alpha(S)=\max \left\{\operatorname{wind}\left(\varphi_{k}(1)\right) ; \gamma_{k}(1)<0\right\}$. Como $\widetilde{\mu}_{C Z}^{1}(S)=3$ temos necessariamente $\alpha(S)=$ $p(S)=1$. Pela definição de $\alpha(S)$ temos $\operatorname{wind}(e(t)) \leq \alpha(S)=1$. Isso implica então que:

$$
\arg (z \bar{e}(T))-\arg (z \bar{e}(0)) \geq(2 \pi+\rho)-2 \pi=\rho,
$$

Tomamos então $n$ um inteiro maior que $\frac{3 \pi}{\rho}$. Seja $\tilde{T}=n T$, concluímos o lema nesse caso pois:

$$
\arg (z \bar{e}(\tilde{T}))-\arg (z \bar{e}(0)) \geq n(\rho)>3 \pi
$$

Caso 2)

Nesse caso, tomando $z(0)$ como um dos autovalores de $A(T)$. Pelo fato dele ser negativo:

$$
\arg (z(T))-\arg (z(0))=(2 k+1) \pi .
$$

Mas $\widetilde{\mu}_{C Z}^{1}(S)=3$, o que força $k$ ser 1 . Como o intervalo $I(A)$ tem comprimento menor que $\frac{1}{2}$, pelo mesmo raciocínio exposto no caso 1$)$ temos que para um certo $\delta \in(0, \pi)$, toda solução $z(t)$ do fluxo linearizado tem que ter variação de argumento maior que $2 \pi+\delta$ no intervalo $[0, T]$, e que wind $(e(t)) \leq 1$. Se tomarmos $\widetilde{T}=n T$ onde $\mathrm{n}$ é inteiro maior que $\frac{3 \pi}{\delta}$ temos:

$$
\arg (z \bar{e}(\tilde{T}))-\arg (z \bar{e}(0)) \geq n \delta-0>3 \pi
$$

Passamos agora à demonstração no caso em que a órbita $P$ é degenerada.

Observamos que o índice de Conley-Zehnder no caso degenerado continua sendo definido pela fórmula $\widetilde{\mu}_{C Z}^{1}(S)=2 \alpha(S)+p(S)$. Nesse caso tome $z(0)$ como sendo um autovetor de $A(T)$. No caso degenerado a curva $A(t) z(0)$ é uma autofunção do operador $L_{S}$ associada ao autovalor nulo, o que pode ser checado por um cálculo direto. Como $p(S)=1$, pois o índice de Conley-Zehnder é ímpar temos necessariamente $\operatorname{wind}(A(t) z(0))>1$, logo a variação de argumento de uma solução do fluxo linearizado no intervalo $[0, T]$ é maior ou igual a $4 \pi$. Como o intervalo $I(A)$ tem comprimento menor que $\frac{1}{2}$ isso garante que a variação de argumento de toda $z(t)$ tem de ser maior que $3 \pi$. Combinando isso como o fato de que $\operatorname{wind}(e(t)) \leq 1$, temos:

$$
\arg (z \bar{e}(T))-\arg (z \bar{e}(0))>3 \pi-2 \pi=\pi,
$$

Basta agora definir $\tilde{T}=3 T$ que vale:

$$
\arg (z \bar{e}(\tilde{T}))-\arg (z \bar{e}(0))>3 \pi .
$$

Isso finaliza a demonstração do lema e portanto também do teorema. 


\subsection{Propriedades e consequências da seção global}

A existência de uma seção global $D$ para o fluxo é um fato muito importante para o estudo da dinâmica de $X_{\lambda}$. Ele se reduz ao estudo da dinâmica de um difeomorfismo de $D$ em $D$. Esse difeomorfismo é chamado de aplicação de primeiro retorno de Poincaré, e é definido da seguinte maneira:

Seja $x \in D$. Defina $\tau(x)$ como sendo o menor tempo positivo no qual $\phi^{\tau(x)}(x) \in D$. Uma aplicação do Teorema da Função Implícita garante que $\tau(x)$ é diferenciável quando o campo $X_{\lambda}$ é transversal a $D$. Segue então da diferenciabilidade do fluxo em relação às condições iniciais que $F: D \rightarrow D:$

$$
F(x)=\phi^{\tau(x)}(x)
$$

é diferenciável.

O fato de $D=u(\mathbb{C})$ ser transversal a $X_{\lambda}$ implica que a projeção ao longo do campo de Reeb $\pi_{x}: T_{x} D \rightarrow \xi_{x}$ é um isomorfismo. Logo a restrição da 2 -forma $d \lambda$ à $D$ é não-degenerada. Para ver isso tomamos 2 vetores $v^{\prime}$ e $w^{\prime}$ em $\xi_{x}$, tais que $d \lambda\left(v^{\prime}, w^{\prime}\right)=1$ e escolhemos $v, w \in T_{x} D$ tais que $\pi_{x}(v)=v^{\prime}$ e $\pi_{x}(w)=w^{\prime}$ (que certamente existem pois $\pi_{x}$ é isomorfismo). Vale a seguinte igualdade:

$$
d \lambda(v, w)=d \lambda\left(\pi_{x}(v), \pi_{x}(w)\right)=d \lambda\left(v^{\prime}, w^{\prime}\right)=1,
$$

que é suficiente para concluir que a restrição $\left.d \lambda\right|_{D}$ é uma forma simplética. Uma característica dessa forma simplética é que ela tem integral finita em $D$ pois:

$$
\left.\int_{\bar{D}} d \lambda\right|_{D}=\int_{\bar{D}} d \lambda=\int_{\partial \bar{D}} d \lambda=\int_{P} \lambda=T
$$

Mostraremos agora o seguinte lema:

Lema 8. F preserva a forma simplética $\left.d \lambda\right|_{D}$

\section{Demonstração:}

$$
\begin{gathered}
D F(x) v=D\left(\phi^{\tau(x)}(x)\right) v=D_{x}\left(\phi^{\tau(x)}(x)\right) v+X_{\lambda}\left(\phi^{\tau(x)}(x)\right) \circ d \tau v= \\
=D_{x} \phi^{\tau(x)}(x) v+d \tau(v) X_{\lambda}\left(\phi^{\tau(x)}(x)\right) .
\end{gathered}
$$

Daí:

$$
\begin{aligned}
& F^{*} d \lambda(v, w)=d \lambda(D F v, D F w)= \\
& \begin{aligned}
=d \lambda\left(D_{x} \phi^{\tau(x)}(x) v+d \tau(v) X_{\lambda}\left(\phi^{\tau(x)}(x)\right), D_{x} \phi^{\tau(x)}(x) w+d \tau(w) X_{\lambda}\left(\phi^{\tau(x)}(x)\right)=\right. \\
=d \lambda\left(D_{x} \phi^{\tau(x)}(x) v, D_{x} \phi^{\tau(x)}(x) w\right)=d \lambda(v, w) .
\end{aligned}
\end{aligned}
$$

onde usamos que $X_{\lambda}$ anula $d \lambda$ e o fluxo $\phi^{t}$ preserva forma $d \lambda$.

Essa $F$ é um difeomorfismo que preserva a forma de área $d \lambda$. Usando um difeomorfismo $G: \mathbb{D} \rightarrow D$, temos $G^{*} d \lambda$ é uma forma simplética em $\mathbb{D}$. Isso implica que $F$ é conjungado a um simplectomorfismo de $\left(\mathbb{D}, G^{*} d \lambda\right)$ pois $\tilde{F}=G^{-1} \circ F \circ G$ preserva $G^{*} d \lambda$.

Pelo fato do espaço $\Omega^{2}\left(\mathbb{R}^{2}\right)$ das funções bilineares anti-simétricas de $\mathbb{R}^{2}$ ser unidimensional, sabemos que toda forma simplética no disco unitário $\mathbb{D}$ é obtida multiplicando-se a forma canônica $d x \wedge d y$ por uma função $f$ não nula. Seja $f: \mathbb{D} \rightarrow \mathbb{R}$ tal que $G^{*} d \lambda=f(x, y) d x \wedge d y$ (no nosso caso suporemos sem perda de generalidade que $f>0$ ). O próximo lema nos mostra que para medidas finitas em $\mathbb{D}$ da forma $s(x, y) d x \wedge d y$ temos apenas um invariante, que é o volume de $\mathbb{D}$ nessa medida. 
Lema 9. Seja $\sigma=f(x, y) d x \wedge d y$ uma forma de área no disco unitário aberto $\mathbb{D}$ com $\int_{\mathbb{D}} f(x, y) d x \wedge$ $d y=\pi$. Então existe um homeomorfismo $h: \mathbb{D} \rightarrow \mathbb{D}$ que leva a medida $d x \wedge d y$ na medida $f(x, y) d x \wedge d y$, ou seja, $\int_{E} f(x, y) d x \wedge d y=\int_{h(E)} d x \wedge d y$ para todo E mensurável . Além disso $h$ tem a mesma diferenciabilidade de $f$ em $\mathbb{D} \backslash\{0\}$.

Demonstração: Inicialmente expressamos nossas duas formas em coordenadas polares $(x, y)=$ $(\sqrt{R} \cos \theta, \sqrt{R} \sin \theta)$. Chamando $f(x(R, \theta), y(R, \theta))=\tilde{f}(R, \theta)$ Obtemos:

$$
\begin{array}{r}
d x \wedge d y=\frac{1}{2} d R \wedge d \theta, \\
f(x, y) d x \wedge d y=\frac{\tilde{f}(R, \theta)}{2} d R \wedge d \theta .
\end{array}
$$

Em coordenadas polares um difeomorfismo $h:(0,1] \times[0,2 \pi] \rightarrow(0,1] \times[0,2 \pi]$ que realize $h^{*} d R \wedge d \theta=\tilde{f}(R, \theta) d R \wedge d \theta$ teria de satisfazer:

$$
\operatorname{det}(D h)=\tilde{f}
$$

Impondo que $h(R, \theta)=(p(R), \vartheta(R, \theta))$, ou seja, que $h$ leva círculos centrados na origem em círculos centrados na origem, a equação (4.24) se torna:

$$
p_{R} \vartheta_{\theta}=\tilde{f} .
$$

Para que $h$ dada acima em coordenadas polares realmente corresponda a uma aplicação em $\mathbb{D}$ temos de exigir periodicidade de $\vartheta$ na variável $\theta$ que é:

$$
\begin{gathered}
\vartheta(R, \theta+2 \pi)=\vartheta(R, \theta)+2 \pi \Leftrightarrow \vartheta(R, \theta+2 \pi)-\vartheta(R, \theta)=2 \pi \Leftrightarrow \\
\Leftrightarrow \int_{0}^{2 \pi} \vartheta_{\theta}(R, \theta) d \theta=2 \pi
\end{gathered}
$$

Da igualdade acima e (4.25) temos $\int_{0}^{2 \pi} p_{r} \vartheta_{\theta} d \theta=\int_{0}^{2 \pi} \tilde{f} d \theta \Rightarrow p_{r}=\frac{1}{2 \pi} \int_{0}^{2 \pi} \tilde{f} d \theta$. Consequentemente (4.25) nos dá $\vartheta_{\theta}=\frac{2 \pi \tilde{f}}{\int_{0}^{2 \pi} \tilde{f} d \theta}$.

Transformamos o nosso problema inicial em um problema de duas equações de primeira ordem que podem ser explicitamente resolvidas por:

$$
\begin{array}{r}
p(R)=\frac{1}{2 \pi} \int_{0}^{R} \int_{0}^{2 \pi} \tilde{f} d \theta d R \\
\vartheta(R, \theta)=\frac{2 \pi \int_{0}^{\theta} \tilde{f} d \theta}{\int_{0}^{2 \pi} \tilde{f} d \theta}
\end{array}
$$

Os fatos:

- $p$ é positiva

$-p(1)=1$ e $\lim _{R \rightarrow 0} p(R)=0$ (para checar a validade dessas 2 fórmulas note que $p(R)$ tem uma interpretação geométrica: $p(R)$ é o volume da bola $B_{R}(0)$ na medida $\tilde{f} d R \wedge d \theta$ dividido por $\left.2 \pi\right)$. Essas duas fórmulas implicam que $p(0,1] \rightarrow(0,1]$ é um homeomorfismo.

-e a periodicidade de $\vartheta$, implicam que $h=(p, \vartheta)$ induz uma aplicação de $\mathbb{D} \backslash\{0\}$.

Como $\vartheta_{\theta}=\frac{2 \pi \tilde{f}}{\int_{0}^{2 \pi} \tilde{f} d \theta}>0, h$ leva o círculo $|z|=R$ no círculo $|z|=p(R)$ de maneira biunívoca. Isso junto com o fato de que $p$ é um homeomorfismo de $(0,1]$ em si mesmo nos garante que $h$ é uma bijeção de $(0,1] \times[0,2 \pi]$ em $(0,1] \times[0,2 \pi]$ e por isso induz uma bijeção de $\mathbb{D} \backslash\{0\}$ em si mesmo.

Notamos ainda que as fórmulas (4.26) e (4.27) nos dão que $h$ é tão diferenciável quanto $f$ se restrito a $\mathbb{D} \backslash\{0\}$. Por último temos que $\lim _{R \rightarrow 0} p(R)=0$ implica que $h$ admite uma extensão 
contínua a $\mathbb{D}$, definindo $h(0)=0$. Para ver que essa extensão é um homeomorfismo de $\mathbb{D}$ em $\mathbb{D}$, observamos que:

-Essa extensão é claramente uma bijeção.

-Como $p^{-1}$ é um homeomorfismo de $(0,1]$ em si mesmo, com $p^{-1}(1)=1$, temos que $p^{-1}$ também satisfaz $\lim _{R \rightarrow 0} p^{-1}(R)=0$

Logo $h^{-1}$ pode igualmente ser estendida de maneira contínua fazendo $h^{-1}(0)=0$. Isso conclui a prova de que $h$ é um homeomorfismo, e por satisfazer a equação 4.25 ele leva $f d x \wedge d y$ em $d x \wedge d y$ em um conjunto de medida total. Isso é suficiente para provar que $h$ transforma $f d x \wedge d y$ em $d x \wedge d y$.

Observação: usando o Lema acima e um resultado de Dacorogna e Moser ([Moser]) é possível achar uma $\tilde{h}$ diferenciável em $\mathbb{D} \operatorname{com} h^{*}(f d x \wedge d y)=d x \wedge d y$.

Exibiremos as importantes consequência do Lema acima. Para simplificar a notação e sem perda de generalidade iremos supor que o período da órbita periódica $P$ é $\pi$ e portanto vale $\int_{D} d \lambda=\pi$.

Nesse caso $h$ transforma $d x \wedge d y$ em $f(x, y) d x \wedge d y$ e o fato de $\tilde{F}$ preservar $f d x \wedge d y$ implica que $h \circ \tilde{F} \circ h^{-1}$ preserva a medida $d x \wedge d y$, pois:

$$
\int_{h \circ \tilde{F} \circ h^{-1}(E)} d x \wedge d y=\int_{\tilde{F} \circ h^{-1}(E)} f(x, y) d x \wedge d y=\int_{h^{-1}(E)} f(x, y) d x \wedge d y=\int_{E} d x \wedge d y
$$

Assim $F$ é conjugado a um homeomorfismo de $\mathbb{D}$ que preserva área. O Teorema da Translação de Brower [5] implica que todo homeomorfismo de $\mathbb{D}$ que preserva área tem um ponto fixo, o que prova a existência de um ponto fixo para $F$. Um importante resultado de John Franks [6] e [7] afirma que se um homeomorfismo de $\mathbb{D}$ que preserva área tem 2 pontos periódicos, então ele tem infinitos pontos periódicos. Como pontos periódicos de $F$ estão em bijeção com as órbitas periódicas para o fluxo de Reeb de $X_{\lambda}$, concluímos com o seguinte teorema:

Teorema 9. Seja $\lambda$ uma forma de contato em $S^{3}$ induzida por uma hipersuperfície extritamente convexa de $\mathbb{R}^{4}$. Então o campo de Reeb $X_{\lambda}$ tem 2 ou infinitas órbitas periódicas.

Em sua versão para fluxos Hamiltonianos esse teorema diz que um nível de energia regular e estritamente convexo em $\mathbb{R}^{4}$ tem 2 ou infinitas órbitas periódicas. 


\section{Apêndice A}

\section{Propriedades geométricas dos planos pseudo-holomorfos}

Neste apêndice nós apresentaremos algumas propriedades geométricas importantes dos planos pseudo-holomorfos que foram usadas em alguns dos argumentos. Essas propriedades são: mostrar que os planos da família $\mu$ são mergulhos o que nos permite aplicar a teoria de Fredholm desenvolvida em [14]; mostrar que o plano $\widetilde{u}$ na simplectização de uma forma $\lambda$ dinamicamente convexa que foi construído no capítulo 3 é um mergulho e é transversal ao campo de Reeb $X_{\lambda}$.

Começamos com a seguinte:

Proposição 10. Seja $\widetilde{u}_{1} \in \mu$. Então $\widetilde{u}_{1}$ é um mergulho.

Demonstração: Nossa prova utilizará o índice de auto-intersecção ind para discos pseudoholomorfos que são mergulhos perto do bordo que foi introduzido em [21]. Um disco pseudo holomorfo $\widetilde{v}: \overline{B_{R}(0)} \rightarrow\left(\mathbb{R} \times S^{3}, \widehat{J}\right)$ é um mergulho perto do bordo se existe $\epsilon>0$ tal que:

$$
\begin{gathered}
\widetilde{v} \mid \overline{B_{R}(0)} \backslash B_{R-\epsilon}(0) \\
\widetilde{v}^{-1}\left(\widetilde{v}\left(\overline{B_{R}(0)} \backslash B_{R-\epsilon}(0)\right)=\overline{B_{R}(0)} \backslash B_{R-\epsilon}(0) .\right.
\end{gathered}
$$

Para discos pseudo-holomorfos que são mergulhos perto do bordo, o índice de auto-intersecção tem a seguinte propriedade:

$-\widetilde{v}$ é mergulho se, e somente se, $i n d(\widetilde{v})=0$.

(A demonstração desse fato baseia-se no fato da chamada positividade de intersecções de curvas pseudo-holomorfas; ver [22, [21]).

Mostraremos primeiramente que para $\widetilde{u}_{1}=\left(a_{1}, u_{1}\right)$ existe $R>0$ tal que $\left.\widetilde{u}_{1}\right|_{\overline{B_{R(0)}}}$ é um mergulho perto do bordo.

Provamos no Teorema 3 que $a_{1}(z) \rightarrow+\infty$ quando $|z| \rightarrow+\infty$ e, pela definição da família $\mu$ temos que $\widetilde{u}_{1}$ é assintótico à órbita periódica $P_{0}$ do elipsóide. Assim $\exists r$ tal que $\left.\widetilde{u}_{1}\right|_{\mathbb{C} \backslash B_{r}(0)}$ é um semicilindro de energia finita na simplectização de $\left(S^{3}, \lambda_{E}\right)$ com a propriedade de que $\left.a_{1}\right|_{\mathbb{C} \backslash B_{r}(0)}(z) \rightarrow$ $+\infty$ quando $|z| \rightarrow+\infty$. Nas coordenadas de Martinet temos, conforme seção 4.1, uma fórmula assintótica para esses cilindros:

$$
\begin{aligned}
& w(s, t)=\left(T s+c+r_{1}(s, t)\right., T t+d+r_{2}(s, t), e^{\int_{s_{0}}^{s} \gamma(\sigma) d \sigma}\left(e(t)+r_{3}(s, t)\right) \in \mathbb{R} \times \mathbb{R} \times \mathbb{D}, \\
&\left|\partial^{\alpha} r_{j}(s, t)\right| \leq M_{\alpha} e^{-d s}, j=1 ; 2, \\
&\left|\partial^{\alpha} r_{3}(s, t)\right| \rightarrow 0 \text { uniformemente em t quando } s \rightarrow+\infty .
\end{aligned}
$$

Lembramos, como foi descrito no capítulo 4 que:

a constante $M_{\alpha}$ depende de $\alpha$; e(t) é um autovetor do operador auto-adjunto $L_{P_{0}}$ (descrito no 
capítulo 2) associado ao fluxo linearizado de $P_{0}(t)$, associado a um autovalor negativo $\gamma$; e a função $\gamma(s) \rightarrow \gamma$ quando $s \rightarrow+\infty$. Essa é uma representação de $\widetilde{u}_{1}$ em coordenadas e:

$$
\Phi \circ w=\widetilde{u}_{1},
$$

onde $\Phi: \mathbb{R} \times \mathbb{R} \times \mathbb{D} \rightarrow \mathbb{R} \times V_{\epsilon}$ é um recobrimento da simplectização de uma vizinhança tubular $V_{\epsilon}$ de $P_{0}$ com a seguinte propriedade:

$$
\begin{aligned}
& \Phi(a, \vartheta, 0)=\left(a, P_{0}\left(T_{0} \vartheta\right)\right), \\
& \Phi(a, \vartheta, z)=\Phi\left(a^{\prime}, \vartheta^{\prime}, z^{\prime}\right) \text { se, e somente se } \\
& a=a^{\prime} \vartheta=\vartheta^{\prime}+2 k \pi ; k \in \mathbb{Z} z=z^{\prime} .
\end{aligned}
$$

Observação A: a existência de um recobrimento com essa propriedade é possível apenas pelo fato de $P_{0}\left(T_{0} t\right) ; t \in[0,1]$ ser uma curva fechada simples, ou equivalentemente o período mínimo de $P_{0}$ ser $T_{0}$. Nesse caso, um resultado de Martinet [Martinet] diz que para alguma vizinhança tubular $V_{\epsilon}$ da órbita $P_{0}$ existe um difeomorfismo $\phi: S^{1} \times \mathbb{D} \rightarrow V_{\epsilon}$ tal que $\phi(\theta, 0)=P_{0}\left(T_{0} t\right)$. Nesse caso definimos $\Phi=\pi \circ \phi$ onde $\pi: \mathbb{R} \times \mathbb{D} \rightarrow S^{1} \times \mathbb{D}$ é o recobrimento usual dado por $\pi(\theta, z)=([\theta], z)$ e $[\theta] \in \mathbb{R} / \mathbb{Z}$.

Pela fórmula assintótica é imediato que para $R^{\prime}$ suficientemente grande $\left.\widetilde{u}_{1}\right|_{\mathbb{C} \backslash B_{R}(0)}$ é uma imersão para todo $R \geq R^{\prime}$. Mostraremos agora que é possível tomar $R^{\prime}$ acima de modo que $\left.\widetilde{u}_{1}\right|_{\mathbb{C} \backslash B_{R}^{\prime}(0)}$ é injetora.

Por absurdo suponha que isso não ocorra para nenhum $R^{\prime}$. Nesse caso seria possível tomar duas sequências $\left(s_{k}, t_{k}\right)$ e $\left(s_{k}^{\prime}, t_{k}^{\prime}\right)$ diferentes, que satisfazendo:

$$
\begin{aligned}
& s_{k}, s_{k}^{\prime} \rightarrow+\infty \\
& t_{k}-t_{k}^{\prime}<2 \pi \\
& \widetilde{u}_{1}\left(e^{2 \pi\left(s_{k}+i t_{k}\right)}\right)=\widetilde{u}_{1}\left(e^{2 \pi\left(s_{k}^{\prime}+i t_{k}^{\prime}\right)}\right)
\end{aligned}
$$

Então usando a fórmula assintótica teríamos:

$$
\begin{aligned}
T_{0}\left(s_{k}-s_{k}^{\prime}\right) & =r_{1}\left(s_{k}^{\prime}, t_{k}^{\prime}\right)-r_{1}\left(s_{k}, t_{k}\right) \\
T_{0}\left(t_{k}-t_{k}^{\prime}\right) & =r_{2}\left(s_{k}^{\prime}, t_{k}^{\prime}\right)-r_{2}\left(s_{k}, t_{k}\right)
\end{aligned}
$$

Usando a desigualdade do valor médio e a equação (A.2), é possível achar $\tilde{M}$ tal que:

$$
\begin{aligned}
\left|r_{j}\left(s_{k}^{\prime}, t_{k}^{\prime}\right)-r_{j}\left(s_{k}, t_{k}\right)\right| & \leq \sup _{|s| \geq \min \left\{s_{k}, s_{k}^{\prime}\right\}}\left(\left|\partial_{s} r_{j}\right|+\left|\partial_{t} r_{j}\right|\right)\left|\left(s_{k}, t_{k}\right)-\left(s_{k}^{\prime}, t_{k}^{\prime}\right)\right| \leq \\
& \leq 2 \tilde{M} e^{-d \min \left\{s_{k}, s_{k}^{\prime}\right\}}\left|\left(s_{k}, t_{k}\right)-\left(s_{k}^{\prime}, t_{k}^{\prime}\right)\right|
\end{aligned}
$$

para $j=1 ; 2$. Agora somando as equações (A.8) e (A.9) obtemos:

$$
\begin{gathered}
\left|T_{0}\left(s_{k}-s_{k}^{\prime}\right)\right|+\left|T_{0}\left(t_{k}-t_{k}^{\prime}\right)\right|=\left|r_{1}\left(s_{k}^{\prime}, t_{k}^{\prime}\right)-r_{1}\left(s_{k}, t_{k}\right)\right|+\left|r_{2}\left(s_{k}^{\prime}, t_{k}^{\prime}\right)-r_{2}\left(s_{k}, t_{k}\right)\right| \leq \\
\leq 4 \tilde{M} e^{-d \min \left\{s_{k}, s_{k}^{\prime}\right\}}\left|\left(s_{k}, t_{k}\right)-\left(s_{k}^{\prime}, t_{k}^{\prime}\right)\right| .
\end{gathered}
$$

Elevando os dois lados da equação acima ao quadrado obteríamos:

$$
\left(T_{0}\right)^{2}\left|\left(s_{k}, t_{k}\right)-\left(s_{k}^{\prime}, t_{k}^{\prime}\right)\right|^{2} \leq 16 \tilde{M} e^{-2 d \min \left\{s_{k}, s_{k}^{\prime}\right\}}\left|\left(s_{k}, t_{k}\right)-\left(s_{k}^{\prime}, t_{k}^{\prime}\right)\right|^{2} .
$$


Mas é possível tomar $R_{1}$ tal que $16 \tilde{M} e^{-2 d R_{1}}<\left(T_{0}\right)^{2}$. Como $s_{k}$ e $s_{k}^{\prime}$ tendem à $+\infty$ existe $k_{0}$ tal que $\min \left\{s_{k}, s_{k}^{\prime}\right\}>R_{1}$ para $k>k_{0}$. Assim para que a equação (A.12) seja válida é preciso valer: $\left|\left(s_{k}, t_{k}\right)-\left(s_{k}^{\prime}, t_{k}^{\prime}\right)\right|^{2}=0$ para $k>k_{0}$ o que implica $\left(s_{k}, t_{k}\right)=\left(s_{k}^{\prime}, t_{k}^{\prime}\right)$ para $k>k_{0}$.

Mas isso contradiz nossa hipótese de que $\left(s_{k}, t_{k}\right) \neq\left(s_{k}^{\prime}, t_{k}^{\prime}\right)$ para todo $k$.

Concluímos então que existe $R^{\prime}$ tal que $\left.\widetilde{u}_{1}\right|_{\mathbb{C} \backslash B_{R}(0)}$ é injetora para todo $R>R^{\prime}$. Como sabemos que $a_{1}(z) \rightarrow+\infty$ quando $|z| \rightarrow+\infty$ é possível tomar $R_{1}>R^{\prime}$ tal que:

$$
\widetilde{u}_{1}\left(\mathbb{C} \backslash B_{R_{1}}(0)\right) \cap \widetilde{u}_{1}\left(B_{R^{\prime}}(0)\right)=\emptyset .
$$

É imediato ver que se tomarmos $R_{0}=R_{1}+50$ o disco pseudo-holomorfo $\widetilde{u}_{1}: B_{R_{0}}(0) \rightarrow\left(\mathbb{R} \times S^{3}, \widehat{J}\right)$ é um mergulho perto do bordo.

Além disso se tomarmos um plano pseudo-holomorfo $\widetilde{u}^{\prime} \in \mu$ suficiente próximo em $\mu$ do plano $\widetilde{u}_{1}$ temos que a restrição $\left.\widetilde{u}^{\prime}\right|_{B_{R_{0}}(0)}$ também é um mergulho pseudo-holomorfo. Isso ocorre porque a convergência de uma sequência de elementos de $\mu$ para $\widetilde{u}_{1}$ implica convergência $C^{1}$ em cada compacto de $\mathbb{C}$. Como perturbações $C^{1}$ suficientemente pequenas de um disco que é mergulho perto do bordo são também mergulhos perto do bordo, temos que perturbações suficientemente pequenas de $\left.\widetilde{u}_{1}\right|_{B_{R_{0}}(0)}$ em $\mu$ também são mergulhos perto do bordo.

Lembramos agora que o plano pseudo-holomorfo $\widetilde{u}_{0} \in \mu$ construído em [1] (com $\left.a_{0}(0)=3\right)$ que usamos para mostrar que a família $\mu$ é não vazia, é um mergulho. Liguemos agora o plano $\widetilde{u}_{0}$ ao plano $\widetilde{u}_{1}$ por um caminho $\widetilde{u}_{c(t)} ; t \in[0,1]$ em $\mu$. Pelo fato do caminho $\widetilde{u}_{c(t)} ; t \in[0,1]$ ser compacto e da observação acima de que se $\left.\widetilde{u}^{\prime}\right|_{B_{R_{0}}(0)}$ é mergulho perto do bordo, para todos $\widetilde{u} \in \mu$ suficiente próximos de $\widetilde{u}^{\prime}$, vale que $\left.\widetilde{u}\right|_{B_{R_{0}}(0)}$ também são mergulhos perto do bordo, segue que existe $R>R_{0}$ tal que:

$\left.\widetilde{u}_{c(t)}\right|_{B_{R}(0)}$ é mergulho perto do bordo para todo $t \in[0,1]$.

O índice de intersecção é um invariante homotópico para mergulhos perto do bordo. Como para $\widetilde{u}_{0}$ temos $\operatorname{ind}\left(\left.\widetilde{u}_{0}\right|_{B_{R}(0)}\right)=0$ pois $\widetilde{u}_{0}$ é um mergulho, temos necessariamente $i n d\left(\left.\widetilde{u}_{c(t)}\right|_{B_{R}(0)}\right)=0$ para todo $t$ o que implica $\left.\widetilde{u}_{1}\right|_{B_{R}(0)}$ é um mergulho. Mas além disso temos:

$$
\widetilde{u}_{1}\left(B_{R}(0)\right) \cap \widetilde{u}_{1}\left(\mathbb{C} \backslash B_{R}(0)\right)=\emptyset .
$$

Para ver isso note que (A.13) implica $\widetilde{u}_{1}\left(\overline{B_{R^{\prime}}(0)}\right) \cap \widetilde{u}_{1}\left(\mathbb{C} \backslash B_{R}(0)\right)=\emptyset$. Por outro lado, como a restrição $\left.\widetilde{u}_{1}\right|_{\mathbb{C} \backslash B_{R^{\prime}}(0)}$ é mergulho, obtemos $\widetilde{u}_{1}\left(\mathbb{C} \backslash B_{R}(0)\right) \cap \widetilde{u}_{1}\left(B_{R}(0)\right) \backslash B_{R^{\prime}}(0)=\emptyset$. Daí segue:

$$
\begin{array}{r}
\emptyset=\left\{\widetilde{u}_{1}\left(\mathbb{C} \backslash B_{R}(0)\right) \cap \widetilde{u}_{1}\left(\overline{B_{R^{\prime}}(0)}\right)\right\} \cup\left\{\widetilde{u}_{1}\left(\mathbb{C} \backslash B_{R}(0)\right) \cap \widetilde{u}_{1}\left(B_{R}(0) \backslash B_{R^{\prime}}(0)\right)\right\}= \\
=\widetilde{u}_{1}\left(\mathbb{C} \backslash B_{R}(0)\right) \cap\left\{\widetilde{u}_{1}\left(\overline{B_{R^{\prime}}(0)}\right) \cup \widetilde{u}_{1}\left(B_{R}(0) \backslash B_{R^{\prime}}(0)\right)\right\}=\widetilde{u}_{1}\left(\mathbb{C} \backslash B_{R}(0)\right) \cap \widetilde{u}_{1}\left(B_{R}(0)\right) .
\end{array}
$$

Concluímos que a fórmula acima, associada ao fato de que $\left.\widetilde{u}_{1}\right|_{B_{R}(0)}$ e $\left.\widetilde{u}_{1}\right|_{\mathbb{C} \backslash B_{R}(0)}$ serem mergulhos, implica que $\widetilde{u}_{1}$ é um mergulho como queríamos demonstrar. $\square$

Proposição 11. Seja $\widetilde{u}$ o plano pseudo-holomorfo na simplectização da variedade de contato dinamicamente convexa $\left(S^{3}, \lambda\right)$ que foi construído no capítulo 3. Então $\widetilde{u}$ é um mergulho.

Demonstração: Por uma análise idêntica à feita na proposição anterior $\exists R>$ tal que.

1- $\left.\widetilde{u}\right|_{\bar{B}_{R}(0)}$ é um mergulho perto do bordo.

$2-\left.\widetilde{u}\right|_{\mathbb{C} \backslash \overline{B_{R}(0)}}$ é um mergulho.

$3-\widetilde{u}\left(\overline{B_{R}(0)}\right) \cap \widetilde{u}\left(\mathbb{C} \backslash B_{R}(0)\right)=\emptyset$.

Lembramos que $\widetilde{u}$ restrito a cada compacto, é obtido como um limite $C^{\infty}$ da sequência $\widetilde{v}_{k}(z)=$ $\left(a_{k}(z)-a_{k}(0), u(z)\right)$ onde:

$$
\left(a_{k}, u_{k}\right)=\widetilde{u}_{k}
$$


e $\widetilde{u}_{k}$ é uma sequência em $\mu$ com $a_{k}(0) \rightarrow-\infty$. Devido à convergência $C^{\infty}$ de $\left.\widetilde{v}_{k}(z)\right|_{\overline{B_{R}(0)}}$ para $\left.\widetilde{u}\right|_{\overline{B_{R}(0)}}$ temos que para $k$ suficientemente grande temos que $\left.\widetilde{v}_{k}(z)\right|_{B_{R}(0)}$ também é um mergulho perto do bordo. Pela invariância homotópica do índice de auto-intersecção de para mergulhos perto do bordo temos que:

$$
\begin{gathered}
\left.\left.\widetilde{v}_{k}(z)\right|_{\overline{B_{R}(0)}} \rightarrow \widetilde{u}\right|_{\overline{B_{R}(0)}} \operatorname{em~} C^{\infty} \text { e } \operatorname{ind}\left(\left.\widetilde{v}_{k}(z)\right|_{\overline{B_{R}(0)}}\right)=0 \forall k \Rightarrow \\
\Rightarrow \operatorname{ind}\left(\left.\widetilde{u}\right|_{\overline{B_{R}(0)}}\right)=0,
\end{gathered}
$$

e, portanto, $\left.\widetilde{u}\right|_{B_{R}(0)}$ é um mergulho. Isso combinado às condições 1,2 e 3 acima prova que $\widetilde{u}$ é um mergulho.

Em primeiro lugar iremos mostrar que como o plano de energia finita $\widetilde{u}$ é um mergulho então a projeção $u$ também é um mergulho. Para ver isso iremos aplicar o teorema da função implícita de [16] (já mencionado interiormente) segundo o qual o plano $\widetilde{u}$ é um elemento de uma família bidimensional de planos pseudo-holomorfos $\widetilde{u}_{x} ; x \in \mathbb{D}$ que folheia uma vizinhança do plano $\widetilde{u}$ em $\mathbb{R} \times S^{3}$. Uma dimensão dessa família é dada pelos planos pseudo-holomorfos $\widetilde{u}_{c}$ dada por:

$$
\widetilde{u}_{c}(z)=(a(z)+c, u(z)) .
$$

Isso implica que existe $c_{0}>0$ tal que $|c|<2 c_{0}$ implica $\widetilde{u}(\mathbb{C}) \cap \widetilde{u}_{c}(\mathbb{C})=\emptyset$. Nosso objetivo é mostrar que isso vale na verdade para qualquer $c^{\prime} \in \mathbb{R}$. Para isso afirmamos inicialmente que $\exists R>0$ tal que para todo $c \in\left[c_{0}, c^{\prime}\right]$ vale:

$$
\widetilde{u}_{c}(z)=\widetilde{u}\left(z^{\prime}\right) \Rightarrow|z|,\left|z^{\prime}\right|<R .
$$

Negando a afirmação teríamos sequências $c_{k}, z_{k}, z_{k}^{\prime}$ satisfazendo:

$$
\begin{gathered}
c_{0} \leq c_{k} \leq c^{\prime},\left|z_{k}\right| \rightarrow+\infty \text { ou }\left|z_{k}^{\prime}\right| \rightarrow+\infty, \\
\widetilde{u}_{c_{k}}\left(z_{k}\right)=\widetilde{u}\left(z_{k}^{\prime}\right) .
\end{gathered}
$$

Observamos inicialmente que na verdade tem de ocorrer $\left|z_{k}\right| \rightarrow+\infty$ e $\left|z_{k}^{\prime}\right| \rightarrow+\infty$. Supondo que $\left|z_{k}\right| \rightarrow+\infty$ temos que a coordenada real de $\widetilde{u}_{c_{k}}\left(z_{k}\right)$ tende a $+\infty$. Se não ocorrer $\left|z_{k}^{\prime}\right| \rightarrow+\infty$, então $z_{k}^{\prime}$ terá uma subsequência limitada, e portanto não poderá ocorrer que a coordenada real de $\widetilde{u}\left(z_{k}^{\prime}\right)$ vá para $+\infty$. Logo, essa diferença de limites contradiz a igualdade $\widetilde{u}_{c_{k}}\left(z_{k}\right)=\widetilde{u}\left(z_{k}^{\prime}\right)$ para todo $k$. Assim $\left|z_{k}\right| \rightarrow+\infty \Rightarrow\left|z_{k}^{\prime}\right| \rightarrow+\infty$. A implicação reversa é provada de maneira análoga, apenas observando que é necessário usar o fato de que $c_{k}$ é limitado.

Como $\left|z_{k}\right| \rightarrow+\infty$ e $\left|z_{k}^{\prime}\right| \rightarrow+\infty$ podemos usar a fórmula assintótica para os planos pseudoholomorfos e obtendo $s_{k}$ e $s_{k}^{\prime}$ por $z_{k}=e^{2 \pi\left(s_{k}+i t_{k}\right)}$ e $z_{k}^{\prime}=e^{2 \pi\left(s_{k}^{\prime}+i t_{k}^{\prime}\right)}$ teríamos:

$$
\begin{gathered}
s_{k}, s_{k}^{\prime} \rightarrow+\infty \\
T_{0}\left(s_{k}-s_{k}^{\prime}\right)+c_{k}=r_{1}\left(s_{k}^{\prime}, t_{k}^{\prime}\right)-r_{1}\left(s_{k}, t_{k}\right), \\
T_{0}\left(t_{k}-t_{k}^{\prime}\right)=r_{1}\left(s_{k}^{\prime}, t_{k}^{\prime}\right)-r_{1}\left(s_{k}, t_{k}\right), \\
e^{\int_{s_{0}}^{s_{k}} \gamma(w) d w}\left(e\left(t_{k}\right)+r_{3}\left(s_{k}, t_{k}\right)\right)=e^{\int_{s_{0}}^{s_{k}} \gamma(w) d w}\left(e\left(t_{k}^{\prime}\right)+r_{3}\left(s_{k}^{\prime}, t_{k}^{\prime}\right)\right) .
\end{gathered}
$$

Notamos inicialmente que usando o fato de que $\left|r_{1}\left(s_{k}^{\prime}, t_{k}^{\prime}\right)-r_{1}\left(s_{k}, t_{k}\right)\right|<2 M\left(e^{-d s_{k}}+e^{-d s_{k}^{\prime}}\right)$ temos que $t_{k}-t_{k}^{\prime}$ tende a 0 e tomando uma subsequência, podemos então assumir que $t_{k}$ e $t_{k}^{\prime}$ convergem a um $t^{*}$. Desejamos mostrar que o mesmo ocorre com $s_{k}-s_{k}^{\prime}$. Argumentando por absurdo, supomos que isso não ocorre. Logo é possível garantir, tomando uma subsequência se 
necessário que existe $\delta>0$ tal que $s_{k}-s_{k}^{\prime}>\delta$ ou $s_{k}^{\prime}-s_{k}>\delta$. Supomos que $s_{k}-s_{k}^{\prime}>\delta$ (sendo o caso $s_{k}^{\prime}-s_{k}>\delta$ idêntico). Caso isso ocorra, temos usando a equação (A.21):

$$
e^{\int_{s_{k}^{\prime}}^{s_{k}} \gamma(w) d w}\left(e\left(t_{k}\right)+r_{3}\left(s_{k}, t_{k}\right)\right)=\left(e\left(t_{k}^{\prime}\right)+r_{3}\left(s_{k}^{\prime}, t_{k}^{\prime}\right)\right) .
$$

Mas usando $s_{k}-s_{k}^{\prime}>\delta$, o fato de que $s_{k}$ e $s_{k}^{\prime}$ tendem a $+\infty$ e $\lim _{s \rightarrow+\infty} \gamma(s)=\gamma<0$ temos que existe $\epsilon>0$ tal que $e^{\int_{s_{k}^{\prime}}^{s_{k}} \gamma(w) d w}<1-\epsilon$. Obtemos então:

$$
\begin{gathered}
\limsup _{k \rightarrow+\infty}\left|e^{\int_{s_{k}^{\prime}}^{s_{k}} \gamma(w) d w}\left(e\left(t_{k}\right)+r_{3}\left(s_{k}, t_{k}\right)\right)\right| \leq(1-\epsilon)\left|e\left(t^{*}\right)\right|= \\
=\limsup _{k \rightarrow+\infty}\left|\left(e\left(t_{k}^{\prime}\right)+r_{3}\left(s_{k}^{\prime}, t_{k}^{\prime}\right)\right)\right|=\left|e\left(t^{*}\right)\right|,
\end{gathered}
$$

o que é absurdo (usamos na equação acima que $s_{k}$ e $s_{k}^{\prime}$ tendem a $+\infty$ e $r_{3}(s, t)$ tende uniformemente a 0 quando $s$ vai para $+\infty$ ). Esse absurdo conclui que $s_{k}-s_{k}^{\prime}$ tende a 0 . Unindo isso à fórmula (A.19) temos:

$$
c_{k}=r_{1}\left(s_{k}^{\prime}, t_{k}^{\prime}\right)-r_{1}\left(s_{k}, t_{k}\right)-T_{0}\left(s_{k}-s_{k}^{\prime}\right)
$$

É imediato que o lado à direita da igualdade acima tende a 0 enquanto o da esquerda não, pois $c_{k}>c_{0}$, logo temos um absurdo.

Tendo estabelecido a validade da fórmula (A.17) prosseguimos na prova do lema. Em [?, 21] é provado que a intersecção entre curvas pseudo-holomorfas é codificada por um índice $\widetilde{i n d}$. Essas intersecções são sempre isoladas, e o índice de intersecção tem a seguinte propriedade:

- se $F$ e $G$ são curvas pseudo-holomorfas então $\widetilde{i n d}(F, G)=0$ se, e somente se, $F$ e $G$ não se intersectam.

Utilizaremos esse índice de intersecção para calcular as intersecções dos discos pseudo-holomorfos $\left.\widetilde{u}_{c}\right|_{B_{R}(0)} ; c \in\left[c_{0}, c^{\prime}\right]$, com o disco $\left.\widetilde{u}\right|_{B_{R}(0)}$. Esse índice tem a propriedade de ser invariante por homotopias. Sabemos que:

$$
\widetilde{\operatorname{ind}}\left(\left.\widetilde{u}_{c_{0}}\right|_{B_{R}(0)},\left.\widetilde{u}\right|_{B_{R}(0)}\right)=0
$$

Pela invariância homotópica concluímos que $\widetilde{\operatorname{ind}}\left(\left.\widetilde{u}_{c^{\prime}}\right|_{B_{R}(0)},\left.\widetilde{u}\right|_{B_{R}(0)}\right)=0$ e isso implica que $\left.\widetilde{u}_{\mathcal{c}^{\prime}}\right|_{B_{R}(0)}$ e $\left.\widetilde{u}\right|_{B_{R}(0)}$ não se intersectam.

Pela fórmula (A.17), concluímos que $\widetilde{u}(\mathbb{C}) \cap \widetilde{u}_{c^{\prime}}(\mathbb{C})=\emptyset$ para todo $c^{\prime} \neq 0$.

Usaremos esse fato para provar agora que $u: \mathbb{C} \rightarrow S^{3}$ é um mergulho.

Teorema 10. O mapa $u: \mathbb{C} \rightarrow S^{3}$ é um mergulho transversal ao fluxo de Reeb.

Demonstração: Para injetividade, notamos que se $u(z)=u\left(z^{\prime}\right)$ seja $c=a(z)-a\left(z^{\prime}\right)$. Então:

$$
\widetilde{u}_{c}(z)=(a(z)+c, u(z))=\left(a\left(z^{\prime}\right), u\left(z^{\prime}\right)\right)=\widetilde{u}\left(z^{\prime}\right) .
$$

Mas como $\widetilde{u}(\mathbb{C}) \cap \widetilde{u}_{c^{\prime}}(\mathbb{C})=\emptyset$ para todo $c^{\prime} \neq 0$, a fórmula acima implica $c=0$. Por $\widetilde{u}$ ser um mergulho tem de valer $z=z^{\prime}$. Concluímos então que $u: \mathbb{C} \rightarrow S^{3}$ é injetora.

Resta provar então:

$u(\mathbb{C})$ é transversal ao campo de Reeb $X_{\lambda}$

Recordamos que em [15] é introduzido um índice $w_{i n d}$ que conta os zeros da projeção $\pi d u$ onde $\pi: T S^{3} \rightarrow \xi$ é a projeção do fibrado tangente de $S^{3}$ na estrutura de contato $\xi$ ao longo do campo de Reeb $X_{\lambda}$. Para um plano pseudo-holomorfo $\widetilde{v}$ este índice tem a seguinte propriedade:

$$
\operatorname{wind}_{\pi}(\widetilde{v})=0 \Leftrightarrow \pi d v(z) \neq 0, \forall z \in \mathbb{C}
$$


Nesse mesmo trabalho é provada a seguinte desigualdade:

$$
0 \leq \operatorname{wind}_{\pi}(u) \leq \frac{1}{2} \mu_{C Z}(\widetilde{u})-1 .
$$

Como $\mu_{C Z}(\widetilde{u})=3$, a desigualdade acima implica $\operatorname{wind}_{\pi}(u)=0$. Sendo assim, temos que para todo $z \in \mathbb{C}$ vale $\pi d v(z) \neq 0$. Como $u$ é a projeção de uma curva pseudo-holomorfa, temos:

$$
\pi d u(z) v \neq 0 \Rightarrow J_{\lambda} \pi d u(z) v \neq 0 \Rightarrow \pi d u(z) i v \neq 0 .
$$

Da fórmula acima temos $\operatorname{ker}(\pi d u(z))=\{0\}$ e portanto $\operatorname{Im}(\pi d u(z))=\xi_{u(z)}$. Isso implica que $\operatorname{Im}(\pi d u(z)) \oplus \mathbb{R} X_{\lambda}(z)=T_{u(z)} S^{3}$ que significa que $u(z)$ é transversal à $X_{\lambda}$, e também que $u$ é um mergulho. 


\section{Referências Bibliográficas}

[1] F. Bourgeois, Y. Eliashberg, H. Hofer, E. Zehnder e K. Wysocki. Compactness results in symplectic field theory, Geometry and Topology Vol 7,(2004), 799-888

[2] B. Dacorogna, J. Moser. On a partial differential equation involving the determinant. Ann. I. H. P. Analyse Non Linéaire, 7, 1-26, 1990.

[3] I. Ekeland. Convexity Methods in Hamiltonian Mechanics, Ergebnisse Math. 19, Springer, Berlin (1989).

[4] Y. Eliashberg, A. Givental e H. Hofer. Introduction to symplectic field theory, GAFA 2000 Special Volume Part II, 560-673.

[5] J. Franks. A new proof of the Brower translation theorem. Erg. Theory. and Dyn. Syst. 12 (1992) $217-226$

[6] J. Franks. Area preserving homeomorphisms of open surfaces of genus 0. Preprint 1995.

[7] J. Franks. Geodesics on $S^{2}$ and periodic points of anullus diffeomorphisms. Invent. Math. 108, (1992), 403-318

[8] M. Gromov. Pseudo-holomorphic curves and symplectic manifolds. Invent. Math. 82, (1985), 307-347

[9] A. Harris, G. P. Paternain, Dynamically convex Finsler metrics and J-holmorphic embedding of asymptotic cylinders, Ann. Global Anal. Geom. 34, 2, (2008), 115-134.

[10] H. Hofer. Pseudo-holomorphic curves in symplectizations with application to the Weinstein conjecture in dimension three, Invent. Math., 114, 515-563.

[11] H. Hofer, E. Zehnder e K. Wysocki. A characterization of the tight three-sphere, Duke Math. Journal, 81,(1995),159-226.

[12] H. Hofer, E. Zehnder e K. Wysocki. Finite energy cylinders of small area, Journal. of Erg. Theory and Dyn. Syst. 22, 5,(2002), 1451-1486

[13] H. Hofer, E. Zehnder e K. Wysocki. Finite energy foliations of tight three-sphere and Hamiltonian dynamics, Ann. of Math. 157, 1,(2003),125-255.

[14] H. Hofer, E. Zehnder e K. Wysocki. Properties of pseudo-holomorphic curves in symplectizations I: Assymptotics, Ann. I. H. P. Analyse Non Linéaire, 13,(1996),337-379.

[15] H. Hofer, E. Zehnder e K. Wysocki. Properties of pseudo-holomorphic curves in symplectizations II: embedding controls and algebraic invariants , GAFA, 5,(1995),270-328.

[16] H. Hofer, E. Zehnder e K. Wysocki. Properties of pseudo-holomorphic curves in symplectizations III: Fredholm theory, Topics in nonlinear analisys, Birkhauser, Basel, (1999),381-475.

[17] H. Hofer, E. Zehnder e K. Wysocki. The dynamics on a strictly convex energy surface in $\mathbb{R}^{4}$, Ann. of Math. (2) 148 (1), (1998), 197-289. 
[18] U. Hryniewicz e P. Salomão. Uma introdução à geometria de contato e aplicações à dinâmica Hamiltoniana , Publicações Matemáticas do Impa, Instituto de Matemática Pura e Aplicada, Rio de Janeiro, 2009. $27^{\circ}$ Colóquio Brasileiro de Matemática.

[19] T. Kato. Perturbation theory for linear operators, Springer-Verlag, New York (1976).

[20] F. Lalonde and D. McDuff. Positive paths in the linear symplectic group. Arnold, Gelfand, Smirnov and Retakh, Editors, Arnold-Gelfand Mathematical Seminars, Geometry and Singularity Theory, Birkhauser (1997),361-388

[21] D. McDuff, The local behaviour of holomorphic curves in almost complex 4-manifolds, J-Diff. Geom. 34, (1991), 143-64.

[22] D. McDuff and D. Salamon, J-holomorphic curves and symplectic topology, American Mathematical Society, Providence, RI, (2004).

[23] P. H. Rabinowitz, Periodic solutions of Hamiltonian systems, Comm. Pure Appl. Math., 31, (2), 157-184.

[24] R. Ruggiero. Dynamics and global geometry of manifolds without conjugate points, Ensaios Matemáticos, Sociedade Brasileira de Matemática, Rio de Janeiro, 2007

[25] A. Weinstein. On the hypotheses of Rabinowitz' periodic orbit theorems, Journal of Diff. Eq., Vol.33, (1979), 353-358 